\section{Small vessel disease}

\section{Small vessel disease}

INCREASED BLOOD-BRAIN PERMEABILITY IN NORMAL-APPEARING WHITE MATTER IN SUBJECTS WITH SMALL VESSEL DISEASE. A MRI STUDY

R. Topakian, T.R. Barrick, R.A. Charlton, F. Schiavone, F.A. Howe, H.S. Markus

Clinical Neuroscience, St. George's University of London, London, United Kingdom

Background: The pathogenesis of cerebral small vessel disease (SVD) is incompletely understood but endothelial dysfunction has been implicated, particularly in SVD with leukoaraiosis. It has been hypothesised that this results in increased blood-brain barrier (BBB) permeability with leakage of blood constituents into the vessel wall and white matter. BBB permeability can be estimated using contrast enhanced magnetic resonance imaging (MRI). We tested the hypothesis that MRI can detect increased BBB permeability in patients with stroke due to SVD compared to age-matched controls.

Methods: 24 patients with symptomatic SVD and 18 controls underwent T1 volumetric MRI before and after injection of $40 \mathrm{ml}$ gadodiamide. Scanning was continued for 32 minutes post injection to determine the time course of contrast enhancement. Pre-contrast T1 images were subtracted from post-contrast images at each time point. Mean signal intensity change was plotted against time to calculate area under curve values. Fluid-attenuated inversion recovery images were used to score leukoaraiosis on the Fazekas scale and to produce a mask containing all white matter lesions (WML). Using the WML mask and high resolution T1 images, the brain was automatically segmented to determine permeability in different compartments: cerebrospinal fluid (CSF), grey matter (GM), normal-appearing white matter (NAWM), and WML.

Results: In both patients and controls, permeability within WML was increased compared to NAWM. Degree of leukoaraiosis was significantly correlated with permeability in CSF $(\mathrm{p}=0.001)$, GM $(\mathrm{p}<0.0005)$, and NAWM ( $\mathrm{p}=0.007)$, but not with permeability in WML $(\mathrm{p}=0.81)$. Multivariate regression analyses identified leukoaraiosis grade as an independent predictor of BBB permeability in NAWM after adjustment for subject status, age, weight, gender, hypertension, diabetes, smoking, and brain atrophy.

Discussion: This study provides evidence of increased BBB permeability in NAWM in SVD. Longitudinal studies are needed to determine whether BBB permeability in NAWM predicts future development of WML.

\section{Small vessel disease}

ON THE ETIOLOGY OF INCIDENT BRAIN LACUNES: LONGITUDINAL OBSERVATIONS FROM THE LADIS STUDY

A.A. Gouw, W.M. van der Flier, L. Pantoni, D. Inzitari, T. Erkinjuntti, L.O. Wahlund, G. Waldemar, R. Schmidt, F. Fazekas, P. Scheltens, F. Barkhof Department of Neurology and Alzheimer Center, Vrije Universiteit Medical Center, Amsterdam, The Netherlands

Background: Lacunes are regarded as subcortical ischaemic infarcts, resulting from the occlusion of small perforating arteries. However, the pathogenetic mechanisms have not been fully elucidated. We studied the relation between incident lacunes and white matter hyperintensities (WMH) in a group of initially independently living elderly over a three year period.

Methods: Baseline and repeat MRI (3-year follow-up) were collected within the multi-center, multinational LADIS study $(\mathrm{n}=358)$. Incident lacunes were characterized with respect to brain region (subcortical white matter (WM), basal ganglia, infratentorial region), their appearance within pre-existent WMH at baseline (yes/no) and (change in) WMH size surrounding the incident lacune.

Results: In total, 106 new lacunes were observed in 62 patients (range 1-8). There were $58(55 \%)$ incident lacunes in the subcortical WM, $35(33 \%)$ in the basal ganglia and $13(12 \%)$ in the infratentorial region. Lacunes in the subcortical WM often occurred (47\%) within pre-existing hyperintensities, in contrast to basal ganglia $(17 \%)$ and infratentorial $(23 \%)$ lacunes $(\mathrm{p}=0.01)$. When lacunes developed outside pre-existing WMH, those located in the subcortical WM were mostly accompanied by newly emerging WMH (71\%), while most of the new basal ganglia $(86 \%)$ and infratentorial incident lacunes (90\%) only had a hyperintense rim or no rim at all $(\mathrm{p}<0.001)$.

Conclusion: Incident lacunes in the subcortical WM seem to be more closely related to WMH than those in the basal ganglia and infratentorial region. We suggest that new lacunes in the subcortical WM develop slowly from ongoing or increasing hypoxia in pre-existing $\mathrm{WMH}$, ultimately leading to cavitation. On the contrary, new basal ganglia or infratentorial lacunes most often appear in previously unaffected tissue, probably as the result of ischemia due to an acute occlusion.

\section{Small vessel disease}

GENELARIZED MICROVASCULAR CHANGES IN PATIENTS WITH CEREBRAL SMALL VESSEL DISEASE: EVIDENCE FROM SUBLINGUAL SIDE-STREAM DARK FIELD (SDF)-IMAGING

M. Khalilzada, K. Dogan, C. Ince, J. Stam

Departments of Neurology and Physiology, Academic Medical Center, University of Amsterdam, Amsterdam, The Netherlands

Background: Cerebral small vessel disease (SVD) is a subtype of stroke with pathologic changes in the cerebral arterioles. It is not known if these changes are limited to the brain, or part of a generalized vascular disorder

Methods: We studied the sublingual microcirculation in 10 healthy controls, 10 patients with large artery strokes (LVD), and 8 patients with SVD (lacunar infarcts, no cardio-embolism or large artery atherosclerosis) by Side-stream Dark Field (SDF)-imaging, using a hand-held SDF microscope connected to a monitor and video recorder. Artifact-free video fragments were analyzed with previously validated methods, by an observer blinded to the origin of the video's. Imaging software measured the length of functional capillaries per surface unit of imaged tissue (functional capillary density, $\mathrm{FCD}, \mathrm{mm} / \mathrm{mm}^{2}$ ). Blood flow was assessed qualitatively using a visual scoring system, and expressed as the percentage of blood vessels with abnormal flow (abnormal flow index, AFI). We also recorded the presence of extravascular erythrocyte material (EEM) as possible evidence of past microbleeds or obliterated vessels. Differences were analyzed with the Kruskal-Wallis test.

Results: FCD differed between the three groups (SVD; LVD; controls: mean 14.8; $17.0 ; 16.1 \mathrm{~mm} / \mathrm{mm}^{2} ; \mathrm{P}=0.01$ ). AFI was significantly higher for SVD patients compared with LVD patients and controls (median $10.2 \% ; 5.4 \%$; $4.4 \%$, respectively; $\mathrm{P}$ 0.04). EEM was more frequent in patients with SVD (median 23\%; $0 \%$; $0 \%$; $\mathrm{P}=0.004)$.

Discussion: We found evidence of pathologic changes in the sublingual microcirculation in patients with SVD, in excess to LVD patients and healthy controls. This is consistent with the hypothesis that cerebral small vessel disease is part of a generalized disorder of the microcirculation.

\section{Small vessel disease}

AGE-RELATED WHITE MATTER CHANGES PROGRESSION OVER TIME AND DISABILITY TRANSITION. THE LADIS (LEUKOARAIOSIS AND DISABILITY) STUDY

A. Poggesi, A. Gouw, G. Pracucci, T. Erkinjuntti, F. Fazekas, J.M. Ferro, M.G. Hennerici, P. Scheltens, D. Inzitari, L. Pantoni, on behalf of the LADIS group

Department of Neurological and Psychiatric Sciences, University of Florence, Florence, Italy

Objective: Our aim was to establish the role of ARWMC progression on the transition from functional autonomy to disability in a cohort of initially non-disabled elderly subjects. 
Methods: The LADIS (Leukoaraiosis And DISability) Study is a longitudinal observational study that involves 11 European centers. Six-hundred-thirty-nine subjects with no or only mild disability according to the Instrumental Activities of Daily Living (IADL) Scale, and with ARWMC of different severity degrees were assessed and followed up for 3 years. The main outcome was transition from 0 or 1 to 2 or more IADL activities limited. ARWMC severity was scored on baseline MRI by the Fazekas scale (mild, moderate, severe group); ARWMC progression after 3 years was assessed with the Rotterdam Progression scale (absence/presence of progression in 9 brain regions).

Results: Out of the 639 subjects enrolled (mean age 74.1 $\pm 5.0, \mathrm{M} / \mathrm{F}: 288 / 351$ ), 391 had both MRI scan and IADL status reassessed at the 3-year follow-up (mean age $73.6 \pm 5.0, \mathrm{M} / \mathrm{F}: 183 / 208$ ). Disability transition occurred in $26 \%$ of these subjects. In a logistic regression analysis, adjusting for other possible determinants of disability, ARWMC progression turned out to predict disability transition (OR 1.37, 95\%CI 1.15-1.63 for each point progression). When baseline ARWMC severity was entered in the model, both ARWMC progression and baseline severity retained an independent impact on disability transition (Rotterdam Progression scale: OR 1.27, 95\% CI 1.05-1.54; Fazekas scale severe vs. mild: OR 3.83, 95\% CI 1.69-8.66). Discussion: In our cohort of initially non-disabled subjects, both ARWMC progression and baseline severity were independent predictors of functional decline.

\section{Small vessel disease}

PROGRESSIVE MOTOR COMPROMISE IS CLOSELY RELATED TO DISABILITY AND DEATH IN AGE-RELATED WHITE MATTER CHANGES 3-YEAR RESULTS OF THE LADIS STUDY

H. Bäzner, C. Blahak, L. Pantoni, D. Inzitari, M.G. Hennerici, on behalf of the LADIS study group

Universitätsklinikum Mannheim, University of Heidelberg, Mannheim, Germany

Background: In the LADIS (Leukoaraiosis And DISability) study eleven European centres collaborated to evaluate age-related white matter changes (ARWMC) as an independent predictor of the transition to disability in the elderly. We aimed at prospectively determining the association between clinical measures of gait and balance with the attainment of the primary study endpoint (transition to $\geq 2$ IADL activities limited or death) at 3-year follow-up in the LADIS population.

Methods: 639 non-disabled individuals were followed-up for 3 years. According to central MRI ratings, patients were stratified into three groups of mild, moderate, and severe ARWMC. The short physical performance battery (SPPB) was performed to assess standing balance, walking speed, and repeated chair stands with total scores ranging from 0-12. A decline in motor performance was defined as a score difference of $\geq 1$ point in SPPB at 3-year follow-up.

Results: At 3-year follow-up data of 457 individuals with complete SPPB scores demonstrated a progressive worsening of SPPB scores in all three ARWMC severity groups (baseline data in brackets): $9.8 \pm 2.4(10.3 \pm 2.0, \mathrm{p}<0.001)$ in the mild, $9.4 \pm 2.4(10.0 \pm 2.0, \mathrm{p}<0.001)$ in the moderate, and significantly worse with a score of $7.6 \pm 3.4(9.2 \pm 2.5, \mathrm{p}<0.001)$ in the severe group. 128 individuals $(20 \%)$ showed a decline $\geq 1$ score point in SPPB which was correlated with a high risk of transition to disability or death within the 3-year follow-up (OR: $3.2,95 \% \mathrm{CI}$ 1.9-5.3; $\mathrm{p}<0.001$ in multivariate logistic regression)

Discussion: This large prospective study demonstrates the significant impact of worsening motor performance over a 3-year follow-up period on the transition to disability or death in elderly individuals with ARWMC.

\section{Small vessel disease}

BALANCE DISTURBANCES AND FALLS PREDICT TRANSITION TO DISABILITY IN PATIENTS WITH AGE-RELATED WHITE MATTER CHANGES - LONGITUDINAL RESULTS FROM THE LADIS STUDY

C. Blahak, H. Baezner, L. Pantoni, D. Inzitari, M.G. Hennerici, on behalf of the LADIS Study Group

Universitaetsklinikum Mannheim, University of Heidelberg, Mannheim, Germany

Background: Previous results of the LADIS (Leukoariosis And DISability) study demonstrated a significant association of age-related white matter changes (ARWMC) with falls and balance disturbances. However, the impact of postural control deficits on the transition to disability in patients with ARWMC have not been examined in a prospective study so far

Methods: 639 initially non-disabled individuals (mean age $74.1 \pm 5.0$ ) were followed-up for 3 years with various clinical and functional tests, including scales to assess disability and scores for motor testing. Centralized rating of MRI was performed to quantify the severity of ARWMC.
Results: Transition to disability or death within the three years of follow-up was predicted by severe ARWMC (OR 2.72; 95\% CI 1.84-4.02; $<<0.001$ in multivariate logistic regression), history of falls (OR 1.72; CI 1.19-2.51; $\mathrm{p}=0.004)$ and balance disturbances (OR 2.85; CI 2.01-4.04; $\mathrm{p}<0.001)$ at baseline. The rate of disability after three year follow-up was $77.5 \%$ in patients with severe ARWMC, history of falls and balance disturbances at baseline, in contrast to just $12 \%$ in patients with mild ARWMC and normal postural control ( $\mathrm{p}<0.001$ in Fisher's exact test). Continuous physical activity significantly reduces the risk of transition to disability or death (OR 0.69; CI 0.49-0.96; $\mathrm{p}=0.029$ ), primarily in patients with existing postural control deficits at baseline.

Discussion: These data reveal the significant impact of falls and balance disturbances on transition to disability or death in patients with ARWMC within three years. However, continuous physical activity already has a beneficial effect to prevent rapid transition to disability in patients with postural control deficits at baseline.

\section{Small vessel disease}

\section{CEREBRAL WHITE MATTER HYPERINTENSITIES, GAIT AND THE RISK} OF INCIDENT FALLS - A POPULATION-BASED STUDY

V. Srikanth, R. Beare, C.L. Blizzard, T.G. Phan, J. Chen, J.M. Stapleton,

M. Callisaya, K.L. Martin, D.C. Reutens

Monash University, Melbourne, Australia

Background: Although cerebral white matter hyperintensities are postulated to cause falls in older people, this has not been demonstrated prospectively.

Methods: We obtained the volume of white matter hyperintensities (WMHV) on magnetic resonance imaging (MRI), computerized gait measurements and physiological measures of falls risk in a randomly selected population-based sample ( $\mathrm{n}$ $=294$, mean age 72.3 years). Incident falls were recorded prospectively over a 12 month period. Using regression modelling, we estimated the risk of incident falls associated with WMHV and examined the relative contributions of WMHV, gait and other physiological factors to falls risk.

Results: Increasing baseline WMHV was associated with any incident fall $(\mathrm{p}=$ $0.008)$ and multiple falls $(p=0.01)$ after adjusting for age, sex, total brain volume and other physiological predictors of falls. Greater WMHV was associated with poorer gait and greater gait variability (both $p<0.001$ ). The effect of WMHV on the risk of falls was magnified in people with poorer quadriceps muscle strength ( $=0.03)$ and greater gait variability $(\mathrm{p}=0.001)$.

Conclusions: These data provide the first evidence supporting a role for cerebral white matter hyperintensities in the causation of falls in the general older population. They should be considered as therapeutic targets for interventional trials in falls prevention.

\section{Small vessel disease}

ENLARGED PERIVASCULAR SPACES ARE ASSOCIATED WITH ISCHAEMIC STROKE, INCREASING AGE, WHITE MATTER LESIONS AND HYPERTENSION BUT NOT WITH SUBTYPES OF STROKE

F.N. Doubal, M.S. Dennis, J.M. Wardlaw

University of Edinburgh, Edinburgh, United Kingdom

Introduction: Enlarged perivascular spaces (EPVS) in the basal ganglia (BG) and centrum semiovale (CS) are frequent on brain magnetic resonance imaging (MRI) and often regarded as normal. Their pathophysiology and clinical significance are uncertain. We hypothesised that if EPVS were related to small vessel disease they would be associated with other features of small vessel disease.

Methods: We prospectively recruited patients presenting to our stroke service with ischaemic stroke. All patients had 1.5T brain MRI. Stroke was subtyped as cortical or lacunar by an experienced stroke physician on clinical and radiological criteria. 100 healthy age matched controls were recruited from the community. Blind to clinical data, a neuroradiologist rated EPVS $0-4(0=$ none, $4=>40)$ in the basal ganglia (BG) and centrum semiovale (CS) on T2-weighted MRI, and white matter lesions (WML) on FLAIR with the Fazekas score. We compared EPVS between stroke subtypes and normal controls and assessed associations with hypertension, age and WML.

Results: We recruited 208 patients; 106 lacunar and 102 cortical (mean ages 67 and $71 \mathrm{yrs}$ respect.). Similar proportions had a history of hypertension (lacunar $58 \%$, cortical 67\%) and diabetes (lacunar 20\%, cortical 13\%). There were similar distributions of EPVS scores $(0: 1: 2: 3: 4)$ in the BG for lacunar $(2: 56: 25: 12: 11)$ and cortical $(5: 60: 11: 16: 10)$ strokes - and in the CS for lacunar $(2: 35: 35: 27: 7)$ and cortical (3:41:32:19:7) strokes. EPVS for both stroke subtypes were higher than age-matched normal controls (Chi squared test for trend $\mathrm{p}<0.01$ ), associated with increasing age (ANOVA $\mathrm{p}<0.001$ ), WML load (Spearman's rho $0.55, \mathrm{p}=0.01$ ) and hypertension (BG EPVS only, Chi square test for trend $\mathrm{p}=0.03$ ). 
Conclusions: EPVS are not normal but are associated with stroke, increasing age, WML and hypertension although not with stroke subtype. The exact pathophysiology of EPVS remains unclear but EPVS should be considered when assessing WML and related risk factors.

\section{Small vessel disease}

VALUE OF RETINAL MICROANGIOPATHY AS LEUKOARAIOSIS, LACUNAR INFARCT AND COGNITIVE IMPAIRMENT MARKER

M. Rodríguez-Yáñez, I. Rodríguez, R. Leira, I. Lema, M. Blanco,

M. Castellanos, D. Santos, S. Arias, X. Rodríguez-Osorio, J. Castillo

Hospital Clínico Universitario, Santiago de Compostela, Spain

Background: Retinal vascularization shares embryological and physiological characteristics with brain microcirculation. We study the diagnostic value of retinal microangiopathy disturbances as possible marker of leukoaraiosis, lacunar infarcts and cognitive impairment.

Methods: We included 150 patients (70.9 (9.1) years, 56\% male) with first ischemic stroke between 3 and 6 months from stroke onset and 50 controls (68.3 (8.2) years, $60 \%$ male). Fifty subjects (43 patients and 7 controls) had diabetes type 2 , diagnosed $>1$ year before. Microangiopathy was defined as the presence of two or more signs of microvascular disturbances. Leukoaraiosis was classified according to its intensity. Cognitive impairment was evaluated by Minimental State Examination (MMSE).

Results: Retinal microangiopathy was associated with higher relative risk of combined neurological disease (leukoaraiosis and/or lacunar infarct and/or cognitive impairment) of 3.0 (CI 95\% 1.1 to 8.1 ) in all subjects, and of 3.8 (CI 95\% 1.1 to 12.6$)$ in non-diabetic subjects. The degree of retinal microangiopathy was significantly associated with leukoaraiosis intensity and MMSE score. Retinal microangiopathy was associated with the presence of lacunar infarcts (OR 7.1, CI 95\% 1.6 to 30.6), but not with leukoaraiosis (OR 1.5, CI 95\% 0.4 to 6.2 ), nor cognitive impairment (OR 3.0, CI 95\% 0.7 to 13.2) after adjustment by significant variables in univariated analysis. In non-diabetic subjects, this association was maintained for lacunar infarcts (OR 4.4, CI 95\% 1.1 to 27.1) and leukoaraiosis (OD 3.7 , CI $95 \% 1.1$ to 24.2 ), but not for cognitive impairment (OR 2.5, CI 95\% 0.4 to 17.3).

Conclusions: The identification of retinal microangiopathy in a fundoscopic examination is a useful screening tool to stratify patients with risk of suffers leukoaraiosis and/or silent lacunar infarcts, especially in non-diabetic subjects with high vascular risk.

\section{Small vessel disease}

\section{A HOSPITAL-BASED STUDY OF IMPACT FACTORS AND PROGNOSIS OF CHINESE PATIENTS WITH LACUNAR INFARCT \\ B. Wu, M. Liu \\ West China Hospital, Sichuan University, Chnegdu, China}

Objectives: The aim of this study was to investigate the case fatality and functional outcome following a first-ever lacunar infarct, accounting for all stroke, ischemic stroke subtypes (lacunar infarct vs non-lacunar infarct) and relevant prognostic variables.

Methods: All patients with a first-ever stroke within 1-month after stroke onset were registered from 4 Chinese hospital-based prospective registries center between March 2002 and March 2007. We collected stroke risk factors and characteristics and used a cross-sectional follow-up. We analyzed 30-day, 90-day, 180-day, and 1-year case fatality, death and dependency, and stroke recurrence by means of logistic regression analysis. We constructed Kaplan-Meier survival curves, and compared the respective subgroups by means of log-rank tests for significant difference.

Results: A total of 3905 patients with first-ever cerebral infarct were included, of which $1650(42.3 \%)$ were lacunar infarct, 2255 non-lacunar infarct. Age and hypertension were the independent risk factors for lacunar infarct. Case fatality was at all four time points lower in lacunar infarct $(5.3,6.4,7.8$, and $17.4 \%$, respectively), higher in non-lacunar infarct $(9.5,14.3,16.5$, and $20.1 \%$, respectively). Age, diabetes, smoking and the NIHSS score at admission were the independent predictors of 1-year case fatality. Death and dependency was also lower in lacuna infarct patients than that in non-lacunar infarct patients. Recurrent stroke was important death cause.

Conclusions: 1 . This is the first large-scale prospective study for lacunar infarct in China. The ratio of lacunar infarct in China was much higher than that of in western country. 2. Age and hypertension were independent risk factor for lacunar infarct. 3. The prognosis after lacunar infarct is not benign. 4. Age, diabetes, smoking and the NIHSS score at admission were the independent predictors of 1-year case fatality.
5. Stroke recurrence is common in the patents with lacunar infarct. Secondary prevention of lacunar infarct is very important.

\section{Small vessel disease}

IMPAIRED VITALITY OF ENDOTHELIAL PROGENITOR CELLS IN PATIENTS WITH CEREBRAL SMALL VESSEL DISEASE: THE IMPORTANCE OF VITAMIN B12 AND HAPTOGLOBIN PHENOTYPE R.P.W. Rouhl, R.J. van Oostenbrugge, J.G.M.C. Damoiseaux, L.L. Debrus-Palmans, J. Delanghe, J.W. Cohen Tervaert, J. Lodder University Hospital Maastricht, Maastricht, The Netherlands

Background: Earlier, we reported that endothelial progenitor cells (EPC), which are important for the maintenance of endothelial integrity, were less vital in lacunar infarct patients, whereas patient serum attenuated EPC outgrowth. In the present study, we investigate two candidate factors for this effect. 1) Vitamin B12 (Vit B12): deficiency relates to higher TNF- $\alpha$ levels, which in turn induce endothelial dysfunction and lower EPC vitality; and 2) haptoglobin phenotypes (Hpt): the Hpt 1-1 may relate to more severe small vessel disease, an effect that might be mediated by lower EPC vitality.

Methods: In 32 prospectively included first-ever lacunar stroke patients, EPC vitality was assessed using cultures (median counts of EPC clusters per well with interquartile range: c/w; IQR). Vit B12 levels and Hpt were determined using previously described

Methods: For Vit B12, we took the mean concentration in a large group of lacunar stroke patients as a cut-off point.

Results: 13 Patients with Vit B12 < $200 \mathrm{pmol} / 1$ had lower EPC vitality $(28.5 \mathrm{c} / \mathrm{w}$; IQR 38.3) than 19 patients $>200 \mathrm{pmol} / \mathrm{l}(79.8 \mathrm{c} / \mathrm{w}$; IQR 65.1; $=.03)$. Furthermore 8 patients with Hpt $1-1$ had lower EPC vitality (37.9; IQR 45.4) than 21 patients with other Hpt (96.3; IQR 94.6; p=.02)

Conclusion: Vit B12 deficiency as well the Hpt 1-1 phenotype relate to lower EPC vitality in lacunar stroke. Though mechanisms have yet to be determined, these findings could open new strategies for the treatment of patients with lacunar stroke and its consequences.

\section{Small vessel disease}

\section{PLASMA VITAMIN B12 STATUS AND CEREBRAL WHITE MATTER} LESIONS

L.M. de Lau, A.D. Smith, H. Refsum, C. Johnston, M.M. Breteler Erasmus Medical Center, Rotterdam, Rotterdam, The Netherlands

Background: Elevated homocysteine levels have been associated with higher prevalence of cerebral white matter lesions and infarcts, and worse cognitive performance. This raises the question whether factors involved in homocysteine metabolism, such as vitamin B12, are also related to these outcomes. We aimed to examine the association of several markers of vitamin B12 status with cerebral white matter lesions, infarcts, and cognition.

Methods: We evaluated the association of plasma concentrations of vitamin B12, methylmalonic acid, holotranscobalamin, and transcobalamin saturation with cerebral white matter lesions, infarcts and cognitive performance among 1,019 non-demented elderly participants of the population-based Rotterdam Scan Study. Analyses were adjusted for several potential confounders, including homocysteine and folate concentration.

Results: Poorer vitamin B12 status was significantly associated with greater severity of white matter lesions, in particular periventricular white matter lesions, in a concentration-related manner. Adjustment for common vascular risk factors (including blood pressure, smoking and intima media thickness) did not alter the associations. Adjustment for homocysteine and folate modestly weakened the associations. No association was observed for any of the studied markers of vitamin B12 status with presence of brain infarcts and cognitive performance.

Discussion: Our results indicate that vitamin B12 status in the normal range is associated with severity of white matter lesions, especially periventricular lesions. Given the absence of an association with cerebral infarcts, we hypothesize that this association is explained by direct effects on methylation reactions and myelin integrity in the brain. 


\section{Acute stroke: complications and early outcome}

\section{Acute stroke: complications and early outcome}

AN OPERATIONAL DEFINITION OF POST-THROMBOLYSIS SYMPTOMATIC HEMORRHAGIC TRANSFORMATION

P. Renou, C. Rosso, S. Deltour, S. Crozier, Y. Samson

Urgences Cérébrovasculaires, Groupe Hospitalier Pitié-Salpêtrière, Paris, France

Background: Post-thrombolysis hemorrhagic transformations (HT) are classified with various clinical and/or radiological criteria resulting in different rates of severe hemorrhages. Here, we assessed three methods of HT classification by testing their independent association with poor outcome after adjusting for other potential clinical and radiological prognosis variables.

Methods: We compared 124 consecutive stroke patients with any type of HT after MRI-based intravenous rt-PA treatment and 80 control patients without HT treated with the same procedure. All patients had a control MRI after 24 to 48 hours and additional MRI and/or CT in case of clinical deterioration. HT within the first week were classified with three different

Methods: (a) the largest volume measured on any imaging modality, (b) the ECASS classification on the same imaging examination, and (c) a clinical classification as symptomatic hemorrhage, defined as NIHSS increase $>4$ points attributed to hemorrhage by the clinicians. Multivariate statistics were used to determine the level of association with poor prognosis (three months $m R s \geq 4$ ) after adjustment for age, gender, initial NIHSS, glucose level, time to treatment, DWI infarct volume, $\mathrm{ADC}$ value and arterial recanalisation.

Results: Three types of HAT were independently associated with poor outcome: hemorrhage volume greater than $15 \mathrm{ml}$ (adjusted OR: 22, CI: 3-144, p=0,002), parenchymal hematoma ( $\mathrm{PH} 1$ and $\mathrm{PH} 2$ ) (adjusted OR: 8 , CI: 2-34, p=0,004) and symptomatic hemorrhages (adjusted OR: 32 , CI 4-280, $\mathrm{p}=0,002$ ).

Discussion: The three definitions of severe HAT are independently associated with poor outcome. Hemorrhage volume greater than $15 \mathrm{ml}$ or clinical definition of symptomatic hemorrhage may be better predictors than the ECASS classification

\section{Acute stroke: complications and early outcome}

MMP-9-RELATED MECHANISMS OF T-PA-INDUCED HEMORRHAGIC TRANSFORMATION: A GENOMIC, PROTEOMIC AND HISTOLOGICAL ANALYSIS IN HUMAN STROKE

J. Montaner, A. Rosell, E. Cuadrado, I. Fernández-Cadenas, A. del Rio, O. Maisterra, M. Ribó, M. Rubiera, C.A. Molina, J. Alvarez-Sabin Neurovascular Research Lab, Stroke Unit, Vall d'Hebron Hospital, Barcelona, Spain

Introduction: Precise mechanism of t-PA-induced-metalloproteinase-9 (MMP-9) dysregulation causing hemorrhagic transformation (HT) remains to be elucidated before attempting to block it.

Methods: Polymorphisms (16 SNPs) covering whole MMP-9 gene and promote regions were analyzed by SNPlex in 540 t-PA-treated patients. MMP-9 mRNA extracted from white blood cell fraction (RiboPureTM-Blood kit) before t-PA administration and $1 \mathrm{~h}$ post-t-PA was measured by TaqMan rt-PCR protocol $(\mathrm{n}=54)$. MMP-9 was serially measured in plasma samples by ELISA. MMP-9 was analyzed by zymography and immunohistochemistry performed to localize MMP-9 and to assess collagen IV integrity in basal lamina (C-IV) in 5 cases of fatal ischemic strokes with hemorrhagic complications. Laser Capture Microdissection was performed to isolate HT vessels.

Results: One SNP in the MMP-9 gene was associated with HT appearance following $\mathrm{t}-\mathrm{PA}$ treatment $(\mathrm{p}=0.034)$, after a logistic regression adjusted by risk factors. MMP-9 mRNA after t-PA was higher among those with $\mathrm{HT}(\mathrm{p}=0.034)$ and MMP-9 plasma levels peaked 2-3h after t-PA administration $(\mathrm{p}<0.001)$ and were higher in cases with HT $(\mathrm{p}<0.05)$. Brain level of the cleaved MMP-9 85kDa-form was elevated in hemorrhagic compared to non-hemorrhagic and contralateral areas $(\mathrm{p}=0.033$ and $\mathrm{p}<0.0001)$. Strong MMP-9-positive neutrophil infiltration surrounding brain microvessels was associated with severe C-IV degradation and blood extravasation. Microdissection confirmed neutrophils as the main source of MMP-9. Ex-vivo treatment of neutrophils with t-PA showed MMP-9 granules release.

Conclusion: Brain bleedings following thrombolysis are at least in part mediated by t-PA-induced-MMP-9 effects at multiple biological levels converting this pathway in a potentially useful therapeutic target.
3 Acute stroke: complications and early outcome

LEUKOARAIOSIS IS ASSOCIATED WITH RISK OF HAEMORRHAGIC TRANSFORMATION AND POOR OUTCOME IN ISCHAEMIC STROKE: DATA FROM THE 'TINZAPARIN IN ACUTE ISCHAEMIC STROKE TRIAL' (TAIST) G.M. Sare, P.M.W. Bath, L.J. Gray, T. Moulin, F. Woimant, For the TAIST Investigators

Institute of Neuroscience, University of Nottingham, Nottingham, United Kingdom

Introduction: Leukoaraiosis is a chronic condition affecting the deep white matter of the brain. Leukoaraiosis is associated with microbleeds on MRI and bleeding following thrombolysis. However, the risk of haemorrhagic transformation and functional outcome in patients with leukoaraiosis and acute ischaemic stroke is not clear, particularly in the presence of anticoagulation.

Methods: TAIST was a randomised controlled trial assessing the safety and efficacy of tinzaparin versus aspirin in 1,484 patients with acute ischaemic stroke. Patients had a CT brain scan at baseline and after 10 days. Scans were adjudicated for the presence of leukoaraiosis at baseline, and haemorrhagic transformation at day 10. Functional outcome measures (modified Rankin scale) was measured at 180 days. Relationships were adjusted for age, sex, baseline severity, history of hypertension, baseline systolic blood pressure, smoking, time to enrolment and treatment group. Outcome was additionally adjusted for causal stroke subtype.

Results: Patients with leukoaraiosis treated with heparin were not more likely to bleed than those treated with aspirin (odds ratio, OR, $1.11,95 \%$ confidence interval, CI, 0.75-1.66). Patients with leukoaraiosis were significantly more likely to have had a lacunar stroke (OR 1.46, 95\% CI 1.10-1.93) and were less likely to have haemorrhagic transformation on day 10 CT (OR $0.65,95 \%$ CI 0.46-0.93) the latter when controlling for lacunar subtype. Despite this, patients with leukoaraiosis had a worse functional outcome measured by the modified Rankin scale (OR $0.47,95 \%$ CI 0.29-0.80).

Discussion: Leukoaraiosis is not associated with increased haemorrhagic transformation after ischaemic stroke even in the presence of heparin, but is associated with poor functional outcome.

\section{Acute stroke: complications and early outcome}

\section{ANGIOEDEMA ASSOCIATED WITH RTPA TREATMENT FOR ACUTE} ISCHEMIC STROKE

A. Valadas, F. Falcão, T. Melo

Santa Maria Hospital, Lisbon, Portugal

Background: The most feared complication of recombinant tissue plasminogen activator (rtPA) treatment for acute ischemic stroke is haemorrhagic transformation. However, other less frequent complications can be life threatening.

Methods: Patients with acute ischemic stroke admitted at Stroke Unit and treated with rtPA are prospectively registered in a database. All cases with angioedema occurring during or after rtPA treatment were selected and reported in this study. Results: Between June 2003 and December 2007, 170 patients with acute ischemic stroke and treated with rtPA were included in the database and angioedema was found in $6(3,5 \%)$. Five patients had middle cerebral artery stroke, four of which with insular compromise, and 1 had brainstem stroke. Five patients, previously or early during the acute stroke, received angiotensin-converting enzyme inhibitor (ACEi). In 4 cases the swelling began in the tongue (in 2 patients contralateral to the ischemic cerebral hemisphere), 1 case presented with oedema of the face and generalized urticaria and 1 case with cough and dysphonia. In 5 cases angioedema appeared soon after the end of rtPA perfusion and in 1 case during the perfusion. The six patients were treated with histamine antagonists and corticosteroids and in 2 epinephrine was administered. Two patients had severe lingual and oropharyngeal oedema and needed mechanic ventilation. In all cases there was a good response to treatment with total regression of the angioedema.

Discussion: Angioedema is associated with rtPA treatment for acute ischemic stroke in about $4 \%$ of the cases, usually in patients treated previously with ACEi In the current protocols there is no restriction to the use of ACEi and no orolingual vigilance is required during and after rtPA administration. More studies will be needed to evaluate the risk of ACEi use during rtPA treatment and to include safety measures to detect and control angioedema. 
5 Acute stroke: complications and early outcome

CT ANGIOGRAPHY RAPIDLY IDENTIFIES AN ISCHEMIC STROKE POPULATION WITH POOR PROGNOSIS DESPITE THROMBOLYSIS WITHIN 3 HOURS FROM ONSET

V. Puetz, I. Dzialowski, N. Steffenhagen, S. Subramaniam, M.D. Hill, S.B. Coutts, A. Krol, C. O'Reilly, A.M. Demchuk, Calgary CTA Study Group University of Calgary, Calgary Stroke Program, Dresden, Germany

Objective: Thrombus burden and extent of ischemic core impact prognosis in acute stroke. A malignant profile of early brain ischemia has been demonstrated in the DEFUSE trial. We sought to determine if such a malignant profile could be predicted in the first 3 hours from onset in thrombolyzed patients using CT-angiography (CTA).

Methods: We studied consecutive patients (04/02-09/07) with acute anterior circulation ischemic stroke who received CTA before thrombolysis within 3 hours from onset. We assessed the Alberta Stroke Program Early CT Score (ASPECTS) on CTA source images (CTASI) with 3-readers-consensus. For clot burden score (CBS), intracranial anterior circulation arteries were allotted 10 points for contrast opacification on CTA. 2 points each were subtracted for absent contrast opacification in the proximal M1, distal M1 or supraclinoid ICA and 1 point each for M2 branches, A1 or infraclinoid ICA. We analyzed parenchymal hematoma $(\mathrm{PH})$ rates, independent outcome $(\mathrm{mRS} \leq 2)$ and fatal outcome at 3 months for categorized combined CTASI-ASPECTS score and CBS score groups where 0 is worst and 20 is best.

Results: We identified 114 patients (46\% women, mean age $70 \pm 14$ years) who underwent CTA before treatment with tPA within 3 hours from onset. Mean onset-to-tPA time was $125 \pm 34$ minutes. 22 patients received additional intraarterial thrombolysis. Patients with extensive early hypocontrastion on CTASI and/or extensive clot burden (combined CTASI-ASPECTS and CBS score $<10$ ) had $50 \%$ $(12 / 24)$ mortality and only $4 \%(1 / 24)$ were functionally independent at 3 months. Of patients with less affected scores (combined CTASI ASPECTS and CBS score $11-20), 57 \%(51 / 90)$ were functionally independent and $10 \%$ (9/90) were deceased. $\mathrm{PH}$ rates were $30 \%$ vs. $8 \%$ (combined score $\leq 10$ vs. 11-20), respectively.

Conclusion: Rapidly obtainable CTA information combining infarct core exten (CTASI ASPECTS) and thrombus burden (CBS) identifies a large hyperacute stroke population $(21 \%)$ who have a very high mortality and very low likelihood of good outcome if thrombolyzed within 3 hours.

6 Acute stroke: complications and early outcome

RECURRENT EVENTS IN TIA AND MINOR STROKE: WHAT EVENTS ARE HAPPENING AND TO WHICH PATIENTS?

S.B. Coutts, M.D. Hill, C.R. Campos, Y.B. Choi, S. Subramaniam, J.C. Kosior, A.M. Demchuk, for the VISION study group

University of Calgary, Calgary, Canada

Background: The risk of a recurrent stroke following TIA or minor stroke is high. Clinical trials are needed to assess acute treatment options in these patients. We sought to evaluate the type of recurrent events and to identify which subsets of patients are at risk for recurrent events.

Methods: 180 patients with TIA or minor stroke were examined within 12 hours and underwent brain MRI within 24 hours. 162 patients had a follow up MRI at 30 days. Any neurological deterioration was recorded and a combination of clinical and MRI factors were used to create a combined event classification. Subgroups of patients analyzed included classical TIA, patients with NIHSS $=0$, and patients with NIHSS $>0$ in Emergency Department (ED).

Results: Overall there were 38 events in 36 patients (20\% event rate); 20 were symptomatic and 18 were silent (only evident because of the follow up MRI). $18 / 20(90 \%)$ symptomatic events were associated with progression of presenting symptoms, compared to $2 / 20(10 \%)$ with a clear recurrent stroke distinct from the original event. We found a low risk of recurrent stroke among classical definition TIA patients $(1.1 \%)$. Patients with an NIHSS $=0$ in the $\mathrm{ED}$, had an intermediate event rate $(6.6 \%)$ between TIA (classical $-1.1 \%)$ and NIHSS $>0(14.4 \%)\left(\chi^{2}\right.$ test for trend, $\mathrm{p}=0.02)$. All clinical categories of patient (TIA $(7.4 \%)$, stroke $(12.9 \%)$, NIHSS $=0(10.6 \%)$ and NIHSS $>0(9.2 \%)$ accumulated silent lesions on MRI.

Conclusions: The high proportion of TIA and minor stroke patients who progress from their initial symptoms has not been recognised previously. True recurrent events, in a new location in the brain, after TIA and minor stroke are actually uncommon. A low risk of recurrence was found in patients with classical TIA and those with no neurological deficits on initial assessment. However even patients, who had an extremely low rate of clinically overt events e.g. classical TIA patients, were at risk for accumulating silent events on MRI.

Oral Session

Acute stroke: complications and early

outcome
7 Acute stroke: complications and early outcome

ADP-INDUCED PLATELET AGGREGATION IN ACUTE ISCHEMIC STROKE PATIENTS ON ASPIRIN THERAPY

J.K. Cha, S.-H. Choi, S.W. Kim, M.J. Kang, J.I. Ung

College of Medicine, Dong-A University, Busan, South Korea

Background: Aspirin is an important tool to prevent the progression of acute ischemic stroke. In this study, we evaluated the relationship between the extent of ADP (adenosine diphosphate)-induced platelet aggregation and outcome in acute ischemic stroke patients on aspirin therapy.

Methods: We selected 116 acute ischemic stroke patients who had been prescribed aspirin and evaluated the optically evaluated ADP-induced platelet aggregation test after 5 days of taking it and investigated prognosis of 90 days after ischemic events. For survival analysis, we used Kaplan-Myer curve.

Results: After stratification of the subjected patients by tertiles of ADP induced platelet aggregation, the events rates were $7.1 \%, 12.1 \%$, and $30 \%(\mathrm{p}=0.023)$. In multiple logistic regression analysis, old age over 70 year (OR, 6.99; 95\% CI, 1.45 to $33.71 ; \mathrm{p}=0.001)$, the history of myocardial infarction (OR, 12.33; 95\% CI 1.42 to $100.72 ; \mathrm{p}=0.019)$, and the highest tertile of ADP induced platelet aggregation had independent significance to the risk of primary end points after acute ischemic stroke (OR, 6.67; 95\% CI 1.57 to 28.37 ; $\mathrm{p}=0.010$ ).

Conclusions: This study showed that the increased ADP induced platelet aggre gation under using aspirin is associated with poor outcome after acute ischemic stroke. Further studies will be needed to confirm the effect of ADP induced platele aggregation on the prognosis in acute ischemic stroke patients on aspirin therapy.

\section{Acute stroke: complications and early outcome}

\section{FATIGUE IS ASSOCIATED WITH MEASURES OF LOW DAYTIME BLOOD} PRESSURE IN STROKE AND TIA

J.A. Harbison, S.M. Walsh, R.A. Kenny

Trinity College Dublin, Dublin, Ireland

Fatigue affects up to $72 \%$ of patients following stroke. There is a reported association between idiopathic forms of chronic fatigue and hypotension. We hypothesised that, in patients with stroke or TIA, an association may exist between fatigue and measures of hypotension detected on ambulatory blood pressure (BP) monitoring. Methods: Subjects recruited from a secondary prevention clinic underwent 24 hour ambulatory blood pressure monitoring and completed a Fatigue Severity Scale (FSS).

Results: Fifty-four subjects were included (59\% female, mean age 69 years). Mean FSS was 3.9 and $26(48 \%)$ has a FSS $>4.0$ indicative of significant fatigue. Mean 24-hour BP for all subjects was $137 / 73$ (SD 34/22). There was no significant difference in mean systolic (SBP), diastolic (DBP) or 24-hour mean arterial pressure (MAP) between patients with and those without significant fatigue. Patients with stroke suffered worse fatigue than those with TIA (mean FSS 4.3 vs $3.2 \mathrm{p}=0.014$ ). Subjects with FSS $>4.0$ suffered episodes of more profound daytime hypotension (Mean daytime minimum SBP 103.7 vs $119.5 \mathrm{mmHg} \mathrm{p}=0.007$ daytime minimum MAP 71.1 vs $82.7 \mathrm{mmHg}$, $\mathrm{p}=0.003$ ). FSS correlated with minimum daytime SBP $(\mathrm{r}=-0.337, \mathrm{p}=0.013)$, daytime minimum MAP $(\mathrm{r}=-0.431, \mathrm{p}<0.001)$ and with the pressure difference between 24-hour mean and daytime minimum SBP ( $\mathrm{r}=-0.408$, $\mathrm{p}=0.002)$. On regression analysis presence of stroke $(\mathrm{p}=0.002)$ and MAP pressure difference $(\mathrm{p}<0.001)$ were independently associated with fatigue severity (R2 $=0.345$ ).

Conclusion: In subjects who have suffered a Stroke or TIA, fatigue is associated with measures of hypotension on ambulatory monitoring. Patients with stroke suffered more fatigue than those with TIA.

\section{Acute stroke: complications and early outcome}

\section{STROKE PATIENTS WITH AND WITHOUT EARLY POSTSTROKE} SEIZURES (EPS): DIFFERENCES IN NITRERGIC AND PROTEOLYTIC INDICES IN CEREBROSPINAL FLUID (CSF)

A. Guekht, N. Gulyaeva, I. Khaimovsky, A. Lebedeva, M. Onufriev, L. Brylev, E. Gusev

Russian State Medical University, Inst. of Higher Nervous Activity \& Neurophysiol., RAS, Moscow, Russian Federation

Background and purpose: EPS occur in 5-10\% of all stroke patients and have deleterious impact on stroke outcome. EPS are associated with excessive neuronal excitability. The objective of the study was to compare neurochemical indices related to nitrergic system and major proteases executing neuronal cell death in CSF of patients with and without EPS. 
Methods: Twenty six patients with first-ever acute ischemic stroke were investigated: 14 - with and 12- without EPS. Groups were similar in age, gender, NIHSS values. NOx levels were measured using a fluorescent probe diaminonaphtalene. Proteolytic activities were assayed fluorometrically using specific substrates for caspase-3, calpain, and cathepsin B.

Results: No increase in NO metabolites (NOx) level in CSF characteristic for acute stroke $(5.5 \pm 0.64$ vs. $2.2 \pm 0.43 \mu \mathrm{mol} / \mathrm{l}$ in controls, $\mathrm{P}=0.01)$ could be detected in EPS patients $(2.6 \pm 0,40 \mu \mathrm{mol} / \mathrm{l})$. While no difference in cathepsin B-inhibiting CSF activity could be revealed between two stroke groups, the calpain-inhibiting activity was more expressed in EPS patients $(45.6 \pm 1.9 \%$ of residual activity vs. $56.4 \pm 2.9 \%, \mathrm{P}<0.05$ ), while caspase-3-activating activity was more significant in CSF of patients without EPS $(132.5 \pm 4.7 \%$ vs. $115.5 \pm 3.3, \mathrm{P}<0.03)$

Discussion: The excessive generation of NO characteristic for ischemic brain is regarded as one of significant neuroprotective factors. We assume that EPS are related to the impaired ability of the brain to urgently increase NOS activity in response to an ischemic situation. The CSF of patients with EPS demonstrates higher ability to inhibit major cell death-related proteases (calpain and caspase-3) suggesting a higher risk for ischemia-induced neurodegeneration in these patients. Supported by RHSF grants

10 Acute stroke: complications and early outcome

HYPEROSMOLAR HYPOTHERMIC NORMOGLYCEMIA (H2N) FOR PREVENTING CEREBRAL EDEMA AFTER LARGE HEMISPHERIC INFARCTION - AN ONGOING FEASIBILITY STUDY

K.E. Wartenberg, C. Reichelt, G. Gahn, S.A. Mayer

Carl Gustav Carus University Hospital Dresden, Dresden, Germany; Columbia University, New York, NY, USA

Introduction: Large hemispheric infarction carries a mortality rate of 40-80\%. Despite the introduction of decompressive hemicraniectomy, the medical management of brain edema after large hemispheric infarction is still not satisfactory.

Methods: We treated 34 patients with large hemispheric infarctions between August 2006 and December 2007 with the combination of insulin infusion (target glucose 4.6-6.1 mmol/L), mild hypothermia $\left(33-35^{\circ} \mathrm{C}\right)$ using a surface cooling or intravascular heat exchange device, and hypertonic saline (3\% sodium chloride acetate) at a rate $1 \mathrm{ml} / \mathrm{kg}$ (goal sodium 150-155 mmol/L) within 72 hours of symptom onset. Primary outcome was progression or evolution of the midline shift on computed tomography (CT). Secondary outcome measures were modified Rankin Scale (mRS) at 3 months and complications.

Results: Of the 34 patients 15 had right-sided infarctions and median age was 65.5 (range $38-83$ ) years. Baseline NIHSS was $18.6 \pm 5.3$. H2N was started on average on the day 1 of symptom onset (range 0-4 days); median duration of treatment was 10 (range 3-20) days. Mean septal midline shift was $1.3 \pm 2.9 \mathrm{~mm}$ on admission, peaked at $8.3 \pm 5.8 \mathrm{~mm}$, and was $1.9 \pm 1.7 \mathrm{~mm}$ on discontinuation of the protocol. At 3 months, 17 patients had died; support had been actively withdrawn in 13 cases. The mRS was 1 in 1 patient, 3 in 3 patients, 4 in 2 patients and 5 in 11 patients. Complications included pulmonary edema $(n=25)$, aspiration and ventilator-acquired pneumonia $(n=8)$, tracheobronchitis $(n=17)$, sepsis and septic shock $(n=9)$, urinary tract infection $(n=6)$, atrial fibrillation with rapid ventricular response $(n=11)$, acute renal failure $(n=9)$, coagulopathy $(n=6)$, thrombocytopenia $(n=16)$, leucopenia $(n=4)$, anemia $(n=9)$, and paralytic ileus $(n=2)$.

Conclusions: The combination of mild hypothermia $\left(33-35^{\circ} \mathrm{C}\right)$, infusion of hypertonic saline, and insulin infusion offers a feasible alternative strategy to minimize massive cerebral edema after large hemispheric infarction and needs to be studied in a standardized trial.

\section{Acute stroke: complications and early outcome}

MANAGEMENT AND OUTCOME OF ACUTE STROKE, A STUDY OF 105043 PATIENTS REPORTED TO THE SWEDISH NATIONAL QUALITY REGISTER FOR STROKE CARE (RIKS-STROKE)

A. Terént, S. Åsberg, K. Henriksson, B. Farahmand, K. Asplund, B. Norrving, B. Stegmayr, P.O. Wester, K. Hulter Åsberg

The Stroke Unit at Uppsala University Hospital, Uppsala, Sweden

Background: Stroke unit care has proven superior to care at general wards in randomised controlled trials (RCTs). The number of hospital beds has been reduced, and today stroke patients are admitted to stroke units (SU), general wards (GW), observational units (OU), intensive care units (ICU) and other type of wards (OW). We have compared the long-term survival of stroke patients treated at different ward types in Sweden.

Methods: Stroke patients who were registered in the Swedish National Quality Register for Stroke Care (Riks-Stroke), from January 1st 2001 through December 31st 2005 were followed until January 31st 2007 by linking Riks-Stroke with the Causes of Death Register. Kaplan-Meier analyses were used to estimate survival times and Cox regression to adjust for different case mix.

Results: Sixty per cent of the patients were admitted to SUs, $22.7 \%$ to GWs $11.2 \%$ to OUs, $3.7 \%$ to ICUs and $2.4 \%$ to OWs. The mean survival time in days (95\% CI) was 1432 (1425-1441) in SUs, 1252 (1239-1264) in GWs, 1349 (1331$1367)$ in OUs, $1222(1188-1256)$ in ICUs and 1435 (1396-1473) in OWs. These relationships persisted after adjustment for age, sex, type of stroke (intracerebral haemorrhage, cerebral infarction) and living conditions. The inclusion of other variables, i.e. level of consciousness, vascular risk factors and medication, does not change outcome data.

Discussion: Stroke unit care appears to be associated with better long-term survival than care at other types of wards. The greatest difference was found between Sus and GWs; this is in line with RCTs and previous observations in Riks-Stroke. The difference between Sus and ICUs is not conclusive in the present study and needs to be further explored.

\section{Acute stroke: complications and early outcome}

\section{EFFECT OF INTRAVENOUS THROMBOLYSIS IN ACUTE ISCHAEMIC} STROKE ON OUTCOME IN DAILY PRACTICE; DATA FROM THE PRACTISE STUDY

M. Dirks, L.W. Niessen, P.J. Koudstaal, J.D. van Wijngaarden, R.J. van Oostenbrugge, C.L. Franke, D.W.J. Dippel, on behalf of the Practise Investigators

Erasmus MC, Rotterdam, The Netherlands

Background: Thrombolysis with intravenous thrombolysis has been proven effective for treatment of patients with acute ischaemic stroke randomised clinical trials. In daily practice, the effect of thrombolysis may be less because of co-morbidity, less strict contra-indications and treatment by less experienced doctors. The aim of the current study was to assess the effectiveness of thrombolysis in an unselected observational cohort of patients within the setting of the PRACTISE trial, a multi-centre cluster-randomised trial of high intensity versus regular intensity implementation of thrombolysis for acute ischaemic stroke.

Methods: Data were collected of all consecutive patients admitted within 24 hours from onset of symptoms with acute stroke for 2 years in 12 representative hospitals in the Netherlands. Contra-indications for thrombolysis and protocol violations were assessed. Within the cohort of patients with an ischaemic stroke admitted within 4 hours outcomes were measured using the modified Rankin scale. Data were analysed with a logistic regression model, using the sliding dichotomy approach.

Results: Of the 5517 stroke patients included in the study, 1658 were admitted with an ischaemic stroke within 4 hours from onset, of whom $698(42 \%)$ were treated with thrombolysis; 10 patients were treated with thrombolysis in the presence of contra-indications (1.4\%.) The odds-ratio for improved outcome after thrombolysis was 1.6 (95\% CI 1.3-2.1) after adjustment for age, sex, stroke severity, co-morbidity, and solid contra-indications for thrombolysis.

Discussion: This study confirms that intravenous thrombolysis for acute ischaemic stroke improves outcome also in standard practice, outside the setting of a randomised clinical trial.

\section{Acute stroke: clinical patterns and practise}

\section{Acute stroke: clinical patterns and practise}

\section{MOBILE VERSUS HOSPITAL BASED TELESTROKE SERVICE.} A CONTROLLED ANALYSIS

J. Schenkel, S. Boy, R. Jankovitz, P. Pilz, G. Uyanik, J. Klucken, N. Fehm, H.J. Audebert

Klinikum Munich-Harlaching, Muenchen, Germany

Background: Telemedicine is increasingly used to provide acute stroke expertise for hospitals without full-time neurological services. Teleconsulting via mobile laptop computers may offer more flexibility compared to hospital based services but concerns about quality and technical reliability remain.

Methods: We conducted a controlled trial, allocating hospital based or mobile teleconsulting in a shift-by-shift sequence and evaluating technical parameters, perception of quality and impact on immediate clinical decisions. Both types of telemedicine workstations were equipped with DICOM (Digital Imaging and Communications in Medicine) viewer and videoconference software. The laptop connected via asymmetrical broadband UMTS (universal mobile telecommunication systems) technology with a one-way spoke-to-hub video transmission whereas 
the hospital based device used landline symmetrical telecommunication including a two-way videoconference.

Results: 127 hospital and 96 mobile based teleconsultations were conducted between June 28 and August 26, 2007 without any technical breakdown. The rates per allocated time were similar with 3.8 and 4.0 per day. No significant differences were found for durations of videoconference (mean: $11 \pm 3$ vs. $10 \pm 3$ min, $\mathrm{p}=0.07$ ), DICOM download ( $3 \pm 3$ vs. $4 \pm 3 \mathrm{~min}, \mathrm{p}=0.19$ ) and total duration of teleconsultations ( $44 \pm 19$ vs. $45 \pm 21 \mathrm{~min}, \mathrm{p}=0.98$ ). Perceived sound and video quality was rated worse but this did not affect the ability to make remote clinical decisions like initiating thrombolysis (18 vs. $13 \%$ of all consultations).

Conclusions: Teleconsultation using a laptop workstation and broadband mobile telecommunication is technically stable and allows remote clinical decision making. There remain disadvantages regarding videoconference quality on the hub side and lack of video-transmission to the spoke side.

\section{Acute stroke: clinical patterns and practise}

OUTCOME OF PATIENTS WITH NEGATIVE CT ANGIOGRAPHY TREATED WITH INTRAVENOUS THROMBOLYSIS

R. Mikulik, D. Goldemund, M. Reif, L. Bunt, P. Aulicky, P. Krupa

Masaryk University, Brno, Czech Republic

Background: Stroke patients without evidence of artery occlusion may not be candidates for thrombolytic therapy (TL). In our study, we sought the outcome of patients with negative CT-angiography treated with TL. Given the increasing reliance on imaging technologies, such findings may impact physician's decision to treat stroke.

Methods: Patients treated with intravenous TL within 3 hours from symptom onset for meaningful neurological deficit at baseline, between August 2003 and June 2007, were included. All had documented negative CT-angiography on independent reviews. Outcome measures included modified Rankin score (mRS) at discharge, incidence of intracranial hemorrhage (ECASS classification) and infarction volume on control CT. Predictors of unfavorable outcome (mRS 2-6) were identified by logistic regression.

Results: Altogether, 173 patients received intravenous TL and 138 had CTangiography. In $39(28 \%)$ patients CT-angiography was negative with mean age $71 \pm 10$ year, $16(41 \%)$ females, and median baseline NIHSS 11 . At 3 month, the mRS 0 to 1 was achieved in $18(46 \%)$ patient and $6(15 \%)$ died. Three patients (8\%) suffered from symptomatic parenchymal hemorrhage type 2 . The median infarct volume was 1.5 (IQR 0-37). In multivariate logistic regression analysis, the independent predictors of unfavorable clinical outcome were higher age, (OR 1.15; $95 \%$ CI, 1.02-1.29), and baseline NIHSS $>12$, (OR 19.3; 95\%CI, 1.9-196.6). One patient was diagnosed with encephalitis.

Conclusions: Negative baseline CT-angiography is not infrequent. TL carries a similar risk of ICH as in unselected patient population. Given patients' dim prognosis, indication of thrombolytic therapy seems justified, although etiologies other than stroke should be considered. More studies are needed.

\section{Acute stroke: clinical patterns and practise}

\section{FREQUENCY AND OUTCOME OF PATIENTS WRONGLY TREATED WITH} INTRAVENOUS THROMBOLYSIS

S. Martínez-Ramírez, A. Vidal, L. Querol, M. Marquié, D. Alcolea,

E. Martínez-Hernández, J.-L. Martí-Vilalta, J. Martí-Fàbregas

Hospital de la Santa Creu i Sant Pau, Spain

Background: Patients with stroke-mimic or psychiatric disorder may be wrongly treated with thrombolytics. We review the frequency and outcome of misdiagnosed patients in a series of consecutive patients treated with rt-PA.

Methods: We retrospectively reviewed all patients treated with intravenous rt-PA in the emergency department at our institution within the last 7 years. All patients were diagnosed and treated by a neurologist. We selected those patients with: 1) No evidence of acute infarction in neuroimaging (CT and/or MRI) before or after thrombolysis. 2) No evidence of vascular occlusion (transcranial Doppler or angio-CT) in the suspected symptomatic artery before thrombolysis. 3) An alternative diagnosis for the neurological symptoms and signs. Functional outcome was measured by the Rankin Scale at 3 months. A score of 0 to 1 was considered a favourable outcome.

Results: We treated 199 patients from 2000 to 2007. Mean age was 65 years and 39\% were men. 28 of them (14\%) had no acute ischemic lesion in followup neuroimaging. From this subgroup, only 5 patients $(2.5 \%)$ had no baseline vascular occlusion and an alternative explanation for their symptoms other than stroke, including epilepsy $(n=1)$, encephalitis $(n=1)$, conversion disorder $(n=1)$ or malingering $(n=2)$. None of these patients suffered hemorrhagic complications, and all of them had a favourable outcome at discharge and at the 3 months follow-up.

Discussion: In our series, there was a small risk of misdiagnosis of stroke $(2.5 \%)$ in the emergency room. Patients wrongly treated with rt-PA had an excellent short and long-term outcome.

\section{Acute stroke: clinical patterns and practise}

\section{EPISODIC LONG-TERM MEMORY DEFICITS IN PATIENTS WITH ACUTE} HIPPOCAMPAL STROKE

A. Förster, T. Jäger, A. Gass, R.R. Kern, M.G. Hennerici, K. Szabo Universitätsklinikum Mannheim, University of Heidelberg, Mannheim, Germany

Background: In hippocampal stroke (HS) clinically apparent mnestic syndromes may be missed even in cases with relatively large ischemic lesions. In this study we focused and evaluated systematically episodic verbal and nonverbal long-term memory in HS patients, both known to be mediated by the left and right medial temporal lobes.

Methods: Twenty patients with acute HS (Left:11, Right:9) were tested - in addition to a standardized neurological examination and stroke MRI - with a detailed neuropsychological battery (test for aphasia and neglect, MMSE, Clock drawing test, short term memory, working memory, and episodic long-term memory).

Results: Verbal long-term memory (Rivermead Behavioural Memory Test, RBMT, Auditory Verbal Learning Test, AVLT) test results were mildly (RBMT) o moderately (AVTL) impaired in HS with left hippocampal lesions compared to normative controls. Patients with left HS were significantly worse than patients with right HS who were only slightly below the mean of the normative sample. In the test for nonverbal long-term memory (Rey-Osterrieth Complex Figure Test, $\mathrm{ROCF}$ ) patients with right HS showed moderate impairment compared to normative samples and performed significantly poorer than patients with left HS. All other neuropsychological tests in HS showed mild impairment or results within the normal range.

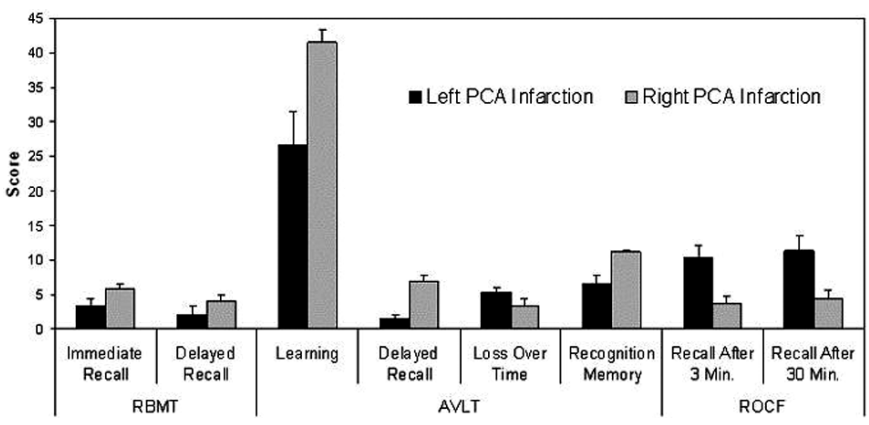

Fig. 1. Performance of 20 patients with left or right PCA infarction and corresponding hippocampa lesions in two tests of verbal episodic long-term (RBMT, AVLT) and one test of nonverbal episodic long-term memory (ROCF). The figure shows means plus standard errors.

Discussion: Detailed neuropsychological testing revealed episodic long-term memory deficits in patients with HS. Consistent with previous knowledge left HS showed deficits in verbal and right HS in non-verbal episodic long term memory, which both should be considered in the evaluation of these patients.

\section{Acute stroke: clinical patterns and practise}

\section{DIGITAL PROBABILISTIC ATLAS OF THE BORDER REGION BETWEEN} THE INTERNAL CAROTID AND POSTERIOR CEREBRAL ARTERIES T.G. Phan, A.F. Fong, G.A. Donnan, V. Srikanth, D.C. Reutens MIND, Clayton, Australia

Background and purpose: The diagnosis of external borderzone infarction is made when the stroke is located at the border between the major cerebral arterial territories (eg angular gyrus). Recent studies have raised questions regarding the location of this borderzone given the variability in the location and extent of the major arterial territories. The concept of distal field perfusion failure suggests that susceptible regions should lie adjacent to areas undergoing infarction due to single artery occlusion. We examined the anatomical distribution of this region.

Methods: Infarcts resulting from occlusion of the ICA or PCA trunk or branches were manually segmented from T2-weighted MR images and linearly registered into a common stereotaxic coordinate space. For each patient with an ICA infarct,

Acute stroke: clinical patterns and practise 
binary maps of voxels in a rim, of varying width surrounding the infarct were created. The maps of individual rims were averaged across subjects to create images of the probability of each voxel lying in the ICA rim. This process was repeated for PCA infarcts to yield a probabilistic atlas of the PCA rim. A map of the probability of each voxel lying concurrently in both rims was created by multiplying the probabilistic ICA and PCA rims together.

Results: There were 39 patients ( 24 males) in the ICA territory group, with a median age of 63 years (range: $26-84$ years), and 30 patients (24 males) in the PCA territory group, with a median age of 61 years (range: 22-86 years). The regions of highest probability in the rim of the ICA and PCA were the optic tract (probability 0.70 ), posterior limb of the internal capsule (probability 0.44 ) and putamen (probability 0.33 ). By contrast, the probabilities of involvement were low $(0.13)$ in the angular gyrus even when the size of the rim was $50 \mathrm{~mm}$.

Conclusion: We have created a digital model of the border region between the ICA and PCA territories which will allow hypotheses relating to distal perfusion failure as a stroke mechanism to be tested.

\section{Acute stroke: clinical patterns and practise}

\section{IS THE ISCHEMIC TOLERANCE IN THE POSTERIOR CIRCULATION HIGHER THAN IN CAROTID TERRITORY?}

J. Pagola, M. Ribo, J. Alvarez-Sabin, M. Rubiera, E. Santamarina, O. Maisterra, R. Delgado-Mederos, C.A. Molina

Unitat Neurovascular, Vall d'Hebron, Barcelona, Spain

Previous studies have suggested that ischemic tolerance in the posterior circulation might be superior that in the anterior territory. We aimed to verify this theory by comparing neurological recovery according to time of ischemia in stroke patients treated with tPA

Methods: Intravenous tPA-treated acute stroke patients with documented middle cerebral artery (MCA) or basilar (BAS) occlusion were studied. TCD assessed recanalization at different time points. According to elapsed time of arterial occlusion, patients were divided in 3 groups: total time of ischemia 0-6 hours, 6-24 hours or $>24$ hours. In order to compare clinical evolution in both groups, neurological improvement was defined as the percentage of recovery at discharge according to baseline NIHSS.

Results: Two hundred and twenty-three patients were studied: 196 MCA, 27 BAO occlusions. Mean time to treatment was longer in BAS patients (231 Vs 172 minutes; $\mathrm{p}=0.031$ ). Early recanalization, within 2 hours after tPA bolus, was more frequent among MCA occlusions (41\% Vs 29\%; p=0.039), and delayed recanalization, 6-24 hours after bolus, more frequent among BAS occlusions (25\% Vs $13 \%$ ); rate of no recanalization at 24 hours was similar in both groups. Clinical recovery according to total time of ischemia was similar in each group: $<6$ hours of ischemia: $(n=8)$ BAS recovered $84 \%$ - $(n=82)$ ACM 69\%; 6-24 ischemia hours: $(\mathrm{n}=7)$ BAS $63 \%$ - $(\mathrm{n}=26)$ ACM $61 \%$; $>24$ ischemia hours $(\mathrm{n}=12)$ BAS $-44 \%$ - $(n=88)$ ACM $11 \%(p=0.23)$. A linear regression model showed that for each additional hour of ischemia ACM patients recovered $-1.78 \%$ and BAS patients $-1.76 \%(\mathrm{p}=0.39)$.

Conclusion: Our data do not support the theory of increased tolerance to ischemia in the posterior circulation territory.

\section{Acute stroke: early management and stroke units}

\section{Acute stroke: early management and stroke units}

STROKE UNIT REFERRALS VIA ANOTHER HOSPITAL REDUCE CHANCES FOR THROMBOLYSIS TREATMENT: ANALYSIS FROM THE AUSTRIAN STROKE UNIT REGISTRY

C. Tatschl, M. Brainin, Y. Teuschl, for the Austrian Stroke Unit Registry Group Center Clinical Neurosciences, Danube University Krems, Tulln, Austria

Background: Successful intervention in acute stroke depends on early arrival at the stroke unit. Aim of our study was to identify time-dependent factors for early treatment on a national level.

Methods: Analysis of the internet-based acute stroke registry concurrently collected by 26 acute stroke units in Austria between January 2003 and February 2007.

Results: Out of 15746 patients with acute stroke, time onset was not known in $6259(40 \%)$ and in further $8768(56 \%)$ arrival was within 24 hours. From those with known onset time the median NIHSS on admission was 4 , median age 72 years.

$84 \%$ were admitted directly to the stroke unit and $16 \%$ were transferred indi- rectly after having been seen at other hospitals. Demographic parameters differed significantly between patients admitted directly to the stroke unit compared to those transferred from other hospitals (median 74 versus 70 years of age, mean pre-stroke Rankin Score 0.74 vs 0.59 ( $\mathrm{p}<0.001)$, women $49 \%$ vs $46 \%(\mathrm{p}<0.05)$, hemorrhagic stroke $9 \%$ vs $14 \%$ and prior stroke $26 \%$ vs $20 \%$; all $\mathrm{p}<0.001$ ).

Among patients transferred directly, 39\% were admitted within 2 hours and $55 \%$ within 3 hours, the respective percentages for indirectly admitted patients being $13 \%$ and $28 \%$.

While the rate of patients admitted within a 2 hours time window improved from 30 to $42 \%$ between 2003 and 2007, the rate of thrombolysis increased from $4.8 \%$ to $9.5 \%, \mathrm{p}<0.001)$.

Cumulative analysis showed that with indirect admission one out of three to four patients misses the time constraints for thrombolysis treatment.

Direct admission increases the odds ratio for thrombolysis treatment (OR 1.8; $95 \%$ CI 1.414-2.207; $\mathrm{p}<0.001$ ) when compared to indirect arrivals.

Conclusion: Acute stroke patients should be admitted directly to a stroke unit, this almost doubles the chances for thrombolysis treatment.

\section{Acute stroke: early management and stroke units}

INFLUENCE OF THE WAY OF TRANSFER TO THE STROKE CENTER OF ACUTE STROKE PATIENTS ON THE RESPONSE TO TRHOMBOLYTIC TREATMENT

N. Pérez de la Ossa, M. Millán, J.F. Arenillas, J. Sánchez-Ojanguren, E. Palomeras, L. Dorado, C. Guerrero, A. Dávalos

Hospital Universitari Germans Trias i Pujol, Badalona, Barcelona, Spain

Background: Acute stroke patients can be transferred directly to the Stroke Center (SC) or may be first attended by another medical professional at community hospitals before their transfer to the SC, where acute stroke expertise is provided $24 \mathrm{~h} / 7 \mathrm{~d}$ and the thrombolytic treatment is administered. Our aim was to analyse the influence of the way of transfer to the SC on the response to thrombolytic treatment and outcome of patients with ischemic stroke.

Patients and methods: We prospectively registered ischemic stroke patients treated with thrombolytic treatment between Jan-05 and Dec-07. The primary outcome variable was good functional outcome at 90 days (Rankin Scale, mRS 2). Secondary outcomes were neurologic improvement (4 in NIHSS score or NIHSS $0-1$ at $24 \mathrm{~h}$ ) and symptomatic hemorrhage.

Results: 153 patients were studied; 45 patients $(29.5 \%)$ received first medical attention at another medical center. Median time from onset to thrombolytic treatment was shorter in patients directly transferred to the SC (135 min vs. 165 $\min ; \mathrm{p}<0,001)$ and stroke severity was higher ( 12 vs $9 ; \mathrm{p}=0.017)$. Patients directly transferred had higher frequency of neurologic improvement at $24 \mathrm{~h}(59,3 \%$ vs $37,2 \% ; \mathrm{p}=0.014)$ and lower symptomatic hemorrhage $(4,7 \%$ vs $14 \% ; \mathrm{p}=0.04)$. With respect to patients first attended into another medical center, direct transfer to the SC was associated with an odds of 2.48 (95\% CI, 1.04-5,8; $\mathrm{p}=0,039)$ of good outcome after adjustment for stroke severity at baseline, atrial fibrillation and baseline glycaemia.

Conclusions: The direct transfer of ischemic stroke patients to the SC is associated with a lower onset to treatment time, better response to thrombolytic treatment and better outcome in comparison with a first level of care in community hospitals.

\section{Acute stroke: early management and stroke units}

\section{RELATIONSHIP BETWEEN STROKE SERVICE CHARACTERISTICS AND} ONSET-TO-CT TIME IN PATIENTS WITH ACUTE ISCHAEMIC STROKE M. Dirks, H.F. Lingsma, J.D. van Wijngaarden, L.W. Niessen, P.J. Koudstaal, R.J. van Oostenbrugge, C.L. Franke, D.W.J. Dippel, on behalf of the PRACTISE investigators

ErasmusMC, Rotterdam, The Netherlands

Background: The number of patients who are eligible for treatment with intravenous thrombolysis is limited because of the narrow time window. Clinical characteristics are known to influence the onset-to-CT time (OCT), but little is known about the influence of stroke service characteristics (SSC): 'time from onset stroke to hospital door' (ODT) and 'time from hospital door to CT' (DCT).

Methods: In a cohort of patients admitted with acute ischaemic stroke within 4 hours from onset of symptoms, data were obtained. SSC data of 12 hospitals were acquired through structured interviews with intra- and extramural representatives, in order to asses 1) protocols and agreements, 2) training and education, and 3) complexity of infrastructure. Data were analysed with multi-level linear regression to adjust for clinical characteristics and centre characteristics.

Results: In total 716 patients were included, 308 (43\%) were treated with thrombolysis. Average DCT of all acute stroke patients admitted within 4 hours from 
onset was 131 minutes (range 20 -360). If the general practitioner had visited the patient first, ODT increased by 25 minutes (95\% CI 18-32). The difference between the most complex stroke service infrastructure and most simple one was $20 \mathrm{~min}$ in ODT (95\% CI 0-40). Within the hospital, a protocol arranging priority for CT scanning saved $10 \mathrm{~min}$ (95\% CI 2-19) of DCT. Having an experienced consultant on call most of the time instead of an inexperienced registrar resulted in a $12 \mathrm{~min}$ (95\% CI 3-21) decrease in DCT.

Discussion: Interventions aimed at simplifying stroke service set-ups and improving clinical pathways may help to shorten onset-to-treatment time in stroke patients and hence may increase the eligibility for thrombolysis.

\section{Acute stroke: early management and stroke units}

\section{BLOOD PRESSURE TREATMENT IN SYSTEMIC THROMBOLYSIS}

B. Dimitrijeski, H.C. Koennecke, A. Hartmann

Charite-Universitätsmedizin Berlin, Campus Benjamin Franklin, Berlin, Germany

Background: Blood pressure (BP) monitoring is essential in patients with ischemic stroke treated with systemic thrombolysis. The guidelines require that their systolic $\mathrm{BP}$ is kept below $185 \mathrm{mmHg}$. However, there is no data confirming that lowering $\mathrm{BP}$ in these patients is safe, especially as cerebral autoregulation may be impaired in these patients.

Methods: A total of 260 patients were treated with systemic thrombolysis within 3 hours according to the NINDS-study criteria. Baseline characteristics and BP at stroke onset was noted. If the systolic BP exceeded $185 \mathrm{mgHg}$, urapidil i.v. was administered according to published guidelines.

Functional outcome at 3-months using the modified Rankin scale was compared between patients who required BP lowering medication and those with systolic BP $<185 \mathrm{mmHg}$ before thrombolysis.

Result: N=52 patients $(20 \%)$ had a systolic BP $>185 \mathrm{mmHg}$ at stroke onset (median $190 \mathrm{mmHg}$ ) and were treated with urapidil intravenously before administering systemic thrombolysis. Unfavourable outcome (Rankin $>2$ at 3 months) occurred in 59\% compared with $47 \%$ in patients with $\mathrm{BP}<185 \mathrm{mmHg}(\mathrm{p}=$ 0.117). Symptomatic intracranial hemorrhage occurred in $2 \%$ and $3.3 \%(\mathrm{p}=0.9)$, and asymptomatic hemorrhage occurred in $23 \%$ and $25 \%$, respectively $(\mathrm{p}=0.9)$.

Conclusion: In patients with ischemic stroke, lowering the systolic BP below the threshold of $185 \mathrm{mmHg}$ with urapidil before the admistration of systemic thrombolysis has no negative effect on functional outcome. There is no increased risk of intracranial hemorrhage in the patients requiring BP lowering before thrombolysis.

\section{Acute stroke: early management and stroke units}

ISOLATED ANTERIOR CEREBRAL ARTERY STROKE: PRESENTATION, LESION, AND OUTCOME IN 30 CONSECUTIVE PATIENTS INVESTIGATED BY DIFFUSION-WEIGHTED MRI (DWI)

D. Ulbricht

Centre Hospitalier Emile Mayrisch, Luxemburg

Introduction: The anterior cerebral artery (ACA) feeds the frontomedian wall, anteroventral parts of the basal ganglia, and anterior $4 / 5$ of the corpus callosum. Ischemic infarctions are rare and account for $0.6-3 \%$ of all territorial strokes. Most knowledge on ACA-stroke stems from few case series and case reports before the advent of neuroimaging.

Patients and methods: We applied routine work-up for ischemic stroke in 26 consecutive patients: MRI including DWI, extracranial ultrasound, ECG, Holter-ECG, transthoracal and transoesophageal echocardiography and EEG. ACA-stroke was clinically suspected and defined by the lesion appearing in DWI. Strokes related to neurosurgical conditions were excluded as were patients carrying significant older lesions.

Results: Mean age was 68 years (range 23-85), 14 were women, 16 men. Acute presentation was akinetic mutism (persistant, $n=3$, brief $n=9$ ), crural hemiparesis $(n=7)$, sudden apathy $(n=1)$, falls with anosognosia for falls $(n=1)$, sudden maladaptive behavior $(n=5)$, amnesia and mutism $(n=1)$, sudden apathy, diarrhea and profuse sweating $(n=1)$, and status epilepticus $(n=1)$. Lesions were left in 17 and right in 12 patients; one had bilateral lesions. Etiology was mainly proximal embolism. One main lesion focus was the anterior cingulate cortex in 18 patients in the territory of the callosomarginal artery, the corpus callosum was affected in 13 patients. Signs of callosal disconnection were observed in 3 patients, and 10/12 patients with callosal lesion remained dependent in everyday life regardless of lesion size. Former daily activities could be resumed in 17 patients.

Discussion: ACA-stroke presented with behavioral trouble and disturbed complex motor integration. Left prefrontal lesions had akinetic mutism of variable duration, their counterpart had acute trouble integrating the representation of the self and the others. Prognosis seems to be worsened by callosal lesions, but otherwise about half of the patients were able to resume their former daily activities.

\section{Acute stroke: early management and stroke units}

CAN THE ORGANISATIONAL COMPONENTS OF STROKE UNIT CARE BE APPLIED TO STROKE CARE POST DISCHARGE? AN OBSERVATIONAL STUDY

A.M. Cox, C.D. Wolfe, C. McKevitt

King's College London, London, United Kingdom

Background: Improving the organisation of acute stroke care has resulted in improved outcomes for patients. Stroke care post discharge can be haphazard and patients report unmet needs. We investigated how organisational components of stroke unit care are operationalised in routine practice and how these might be applied to the organisation of care post discharge.

Methods: We observed care in 3 UK teaching hospital stroke units, using an observational schedule to record organisational components of effective stroke care as identified in the literature, and unstructured fieldnotes. These qualitative data were analysed for emerging themes.

Results: We observed 10 team meetings, 5 meetings between staff members, 2 outpatient clinics, 3 ward rounds and the work of rehabilitation therapy staff (6 days). There were variations in the use of formal organisational procedures including information communication, key working and joint working. In settings with fewer formal structures decision-making was led by groups of key individuals In all settings strong leadership and patterns of hierarchy within and between professions were apparent. All settings held multi-disciplinary meetings but the extent and definition of multi-disciplinary working varied. Professional roles and identities remained distinct. The defined geographical location of the stroke unit facilitated opportunistic discussion about patients, particularly between professions with less formal routes of communication.

Conclusion: Organisation of acute stroke care varied widely in routine practice but geographical collocation facilitated communication. Improving the delivery of community care will require attention to organisational structures and effective leadership, as well as processes through which care is organised.

\section{Epidemiology of stroke I}

\section{Epidemiology of stroke I}

\section{BIRTH WEIGHT AND CHILDHOOD BMI AMONG 191,163 DANISH} SCHOOLCHILDREN AND THE RISK OF ISCHEMIC STROKE IN ADULTHOOD

J. Baker, L. Olsen, T. Truelsen, T.I.A. Sørensen

Institute of Preventive Medicine, Centre for Health and Society, Copenhagen, Denmark

Background: It has been shown that higher childhood BMI raises the risk of coronary heart disease in adulthood (Baker JL, Olsen LW, Sørensen TIA. NEJM 2007;357:2329-37). Although birthweight has been associated with stroke, less is known about how childhood BMI may affect the risk. As stroke subtypes have different etiologies, we focused on ischemic stroke and investigated if birthweight and childhood BMI from 7-13 years of age was associated with ischemic stroke in adulthood.

Methods: 97.391 boys and 93.772 girls born from 1936-1976 in the Copenhagen School Health Records Register were included in the study. BMI values were calculated and transformed to $\mathrm{z}$ scores. Stroke events were obtained from Danish Hospital and Cause of Death Registers. Cox regressions were performed.

Results: Birthweight was associated with ischemic stroke in adulthood among boys and girls. Compared with the reference category $(3251-3750 \mathrm{~g})$ boys in the lowest $(2000-2750 \mathrm{~g})$ had a relative risk (RR) of 1.60 (95\% CI: $1.30-1.98)$ of stroke, and girls in the lowest category had a RR of 1.34 (95\% CI: 1.02-1.75). BMI at 13 years was independently associated with ischemic stroke only in boys. In a model adjusted for birthweight, compared to those with a $\mathrm{z}$ score in the reference category $(-0.25-0.25)$, boys with a BMI z-score $\geq 0.75$ had a RR of 1.35 (95\%CI: $1.10-1.67)$ Conclusion: Birthweight is associated with ischemic stroke in adulthood among boys and girls. BMI at 13 years of age was associated with ischemic stroke in adulthood only among boys. These results suggest that some risk for ischemic stroke is already present at birth but that childhood BMI among boys matters as well; thus prevention possibilities remain

Funding: NIH NRSA F32DK070491 (to JLB) \& the Danish National Research Foundation 


\section{Epidemiology of stroke I}

TRANSITION INTO SUMMER TIME ABRUPTLY SHIFTS THE CIRCADIAN PATTERN OF STROKE ONSET

C. Foerch, H.W. Korf, H. Steinmetz, M. Sitzer

Goethe-University, Frankfurt am Main, Frankfurt am Main, Germany

Background: Stroke onset shows a circadian pattern with highest incidence in morning hours. Diurnal oscillations of blood pressure, hormones and platelet function are supposed to be underlying mechanisms, but exogenous factors like increasing physical activity after awakening and recognition of so far undetected night time strokes may also be of relevance. Transition into Summer Time (ST) and back constitutes an arbitrary interference into the natural time pattern. This study was performed to characterize the effects of the transitions on the circadian pattern of stroke onset.

Methods: Our study relied on a prospective hospital based stroke registry in Germany containing 44251 datasets with admission dates between year 2000 and 2005 and a known time point of stroke onset. To gain a uniform timeline, time points of stroke onset were set back from Central European Summer Time (CEST) to Central European Time (CET) for patients admitted in ST periods.

Results: As compared to the last week prior to the clock change, transition into ST resulted in a drop of the time points of stroke onset in the first week after the clock change in reference to the uniform timeline (i.e. CET; for the 25 th percentile of the diurnal pattern $-60 \mathrm{~min}$, for the 50th percentile $-60 \mathrm{~min}$; Mann Whitney $\mathrm{U} \mathrm{p}<0.001$, patients pooled on a weekly basis). Vice versa, transition from CEST to CET led to increasing stroke onset time points $(+60 \mathrm{~min},+60 \mathrm{~min}$, respectively; $\mathrm{p}=0.001)$. A significant shift was already present at the first and second day after the transitions (i.e. Mon/Tue). A gradual adjustment within the first week was not apparent. Discussion: Transition into ST and back is coupled with sudden shifts of stroke onset time points in reference to the timeline effective prior to the shifts. As clock change is likely to abruptly shift the sleep-wake cycle of most individuals, our results support exogenous factors associated with awakening to be importan determinants of the circadian pattern of stroke onset rather than endogenous factors, as molecular circadian clocks would need at least a few days to gradually adjust.

\section{Epidemiology of stroke I}

PATIENTS WITH CARDIAC, CEREBRAL OR PERIPHERAL ARTERY DISEASE HAVE DIFFERENT VASCULAR RISK FACTORS AND DIFFERENT RISKS OF FUTURE VASCULAR EVENTS

S.W. Achterberg, M.J. Cramer, L.J. Kappelle, G.J. de Borst, Y. van der Graaf, A. Algra, for the SMART study group

University Medical Center Utrecht, Utrecht, The Netherlands

Background: Patients with coronary artery disease (CAD), cerebrovascular disease (CVD), or peripheral artery disease (PAD) have different expressions of atherothrombotic disease. Limited data are available on a direct comparison of their vascular risk factors at baseline and their risk of future vascular events during follow-up

Methods and results: This study involved 3114 consecutive patients from the same hospital based series (the Second Manifestations of ARTerial disease (SMART) study) who were treated because of clinical manifestation of cerebral, cardiac or peripheral atherothrombotic disease,. Prevalence of baseline characteristics was compared between the three SMART subgroups with linear or logistic regression analysis. Primary outcome was the composite of myocardial infarction, stroke and vascular death, The incidence of outcome events was analysed with Cox regression. All analyses were adjusted for age and sex. At baseline, CAD patients were the most obese (BMI $27.3 \mathrm{~kg} / \mathrm{m}^{2}$ ), PAD patients smoked most $(54 \%)$ and had higher prevalence of hypertension and hypercholesterolemia compared with the other groups. CVD and PAD patients had more frequent aortas $>3 \mathrm{~cm}$ than CAD patients (OR 2.21; 95\%CI 1.40-3.47), more often carotid stenosis $>50 \%$ (OR 8.7; 95\%CI 6.8-11.1), more frequent ankle brachial index $<0.90$ (OR 12.8; 95\%CI 10.2-16.0), and more renal impairment (OR 1.46; 95\%CI 1.22-1.74). Patients were followed during a mean of 4.1 years. Primary outcome events were less common in patients with CAD (6.8\% vs. $14.5 \%$ in CVD and $14.1 \%$ in PAD). Patients with PAD had the highest death rate $(3.9 \%$ vs. $3.0 \%$ for CVD and $1.4 \%$ for CAD) and significantly more cardiac events than patients with CAD (HR 1.4; 95\%CI 1.0-1.9). Patients with CAD had striking less major bleeding complications compared with the other two patient groups (HR 0.42; 95\%CI 0.26-0.67)

Conclusions: Patients with different manifestations of atherosclerosis have a different vascular risk factor profile. Prognosis in patients with clinical manifestations of CAD is more benign than in patients with CVD or PAD.

\section{Epidemiology of stroke I}

LONG-TERM APPEARANCE OF LACUNAR INFARCTION ON IMAGING: WHAT PROPORTION BECOME CSF-CONTAINING CAVITIES?

G.M. Potter, F. Doubal, C.A. Jackson, C. Sudlow, M.S. Dennis, J.M. Wardlaw University of Edinburgh, Western General Hospital, Edinbugh, United Kingdom

Introduction: On T2 or FLAIR MR imaging, established lacunar infarcts are often defined as small CSF-containing cavities, but when acute, many lacunar infarcts would be indistinguishable from age-related white matter hyperintensities (WMH) without DWI, especially in older patients. The time taken for acute lacunes to go from resembling a WMH to a cavity, and the proportion that do, is unknown.

Methods: From two studies, we identified all patients with acute lacunar stroke, who had DWI, T2W and FLAIR imaging at presentation and FLAIR/T2 at least two weeks later. The acute infarct on FLAIR/T2 was identified by reference to DWI; its appearance on follow-up FLAIR was classified as resembling WMH/high signal only, or possibly/probably cavitated (ie containing low signal CSF on FLAIR). On the acute scan, we recorded acute infarct size $(\mathrm{mm})$, presence of CSF-containing "old lacunar infarcts", WMH score (Fazekas), and enlarged perivascular spaces (PVS, $<2 \mathrm{~mm}$ on T2WI, on a local scale).

Results: Amongst 30 patients, $8(27 \%)$ showed changes on the follow-up scan consistent with developing cavitation (median 57, range 39-81 days), vs median 53 (range 30-168) days for the $22(73 \%)$ with no cavitation. Average infarct size for patients with cavitation was $12.4 \mathrm{~mm}$ vs $9.5 \mathrm{~mm}$ in those showing no cavitation $(\mathrm{p}=0.13)$. CSF-containing "old lacunar infarcts" were present in $6 / 8$ cavitating vs $9 / 22$ non-cavitating infarcts (Chi squared $\mathrm{p}=0.09$ ). Moderate/severe $\mathrm{WMH}$ and moderate/severe PVS were not more frequent in those with cavitating infarcts (Chi squared $\mathrm{p}=0.77$ and $\mathrm{p}=0.42$ respectively).

Conclusions: Cavitation occurs in $<1 / 3$ of lacunar infarcts within the first $2-3$ months but some may never cavitate and so permanently resemble WMH. Lacunar infarcts which retain the appearance of WMH (up to 2/3) may be overlooked where only CSF-containing cavities are counted as old lacunar infarcts. This has important implications for epidemiology and observational studies of the causes and associations of lacunar stroke.

\section{Epidemiology of stroke I}

INCIDENCE AND SURVIVAL IN SYMPTOMATIC LACUNAR STROKE IN DIJON, FRANCE, FROM 1989 TO 2006: A POPULATION-BASED STUDY

Y. Bejot, G.V. Osseby, M. Caillier, A. Catteau, O. Rouaud, G. Couvreur, J. Durier, T. Moreau, M. Giroud

Dijon Stroke Registry, EA 4184, Dijon, France

Background: Lacunar infarcts are usually regarded as benign, but populationbased studies are required to assess the exact impact of this stroke subtype in cerebrovascular pathology.

Methods: We evaluated incidence and survival in symptomatic lacunar stroke in a prospective well-defined population-based study, in Dijon, France $(150,000$ inhabitants), from 1989 to 2006.

Results: A total of 2536 ischemic strokes were recorded. Among these, 715 (28\%) were lacunar infarcts (354 men and 361 women). From 1989 to 2005, we observed a significant rise in the incidence of lacunar stroke in the two sexes considered together $([R R] 1.02 ; 95 \%$ CI 1.005-1.035; $\mathrm{p}=0.007)$ whereas the variation was not significant in either men or women when considered separately. The incidence significantly increased in patients under 65 years old ([RR] $1.049,95 \%$ CI 1.0175 1.0817; $\mathrm{p}=0.002)$. For lacunar infarcts, survival rates were $96 \%$ at 1 month $(95 \%$ CI $0.94-0.97), 86 \%$ at 1 year (95\% CI $0.83-0.89)$, and $78 \%$ at 2 years $(95 \% \mathrm{CI}$ $0.75-0.81$ ), and were significantly higher than those for non-lacunar stroke (HR $2.05 ; 95 \%$ CI $1.70-2.47, \mathrm{p}<0.001)$

Conclusion: Our results suggest a significant increase in the incidence of lacunar stroke even when any classification biases are taken into account. Even though lacunar stroke is associated with a relatively good short-term prognosis in term of survival, there is a demonstrated risk of reccurence and cognitive dysfunction. Lacunar stroke should therefore not be considered as benign.

\section{Epidemiology of stroke I}

THE EFFECT OF VERY EARLY MOBILISATION ON MOOD AFTER STROKE J.M. Collier, T.B. Cumming, A.G. Thrift, J. Bernhard

National Stroke Research Institute, Melbourne, Victoria, Australia

Background: After stroke, patients spend over 50\% of the day resting in bed Immobility may increase negative mood symptoms, such as depression and anxiety. Increasing mobility early after stroke may have important psychological benefits. 
Aims: To determine the effect of very early mobilisation (VEM) on depression and anxiety after stroke.

Methods: Patients with confirmed stroke admitted within 24 hours of symptom onset were included. Data were collected as part of A Very Early Rehabilitation Trial (AVERT), a multi-centre randomised controlled trial. VEM patients were mobilised earlier (within 24 hours of stroke) and more frequently than standard care (SC) patients. The Irritability, Depression and Anxiety (IDA) scale was administered at 7 days and 12 months post stroke.

Results: Mean age of patients $(\mathrm{n}=71)$ was 74.7 years. At 7 days, VEM patients were less depressed $(\mathrm{P}=0.012)$ and marginally less anxious $(\mathrm{P}=0.073)$ than SC patients. Adjusting for potential confounding factors (age, sex, NIH score, premorbid disability and 7-day walking status), VEM patients was associated with less depression (OR 0.14, 95\% CI 0.03 to $0.61, \mathrm{P}=0.009)$, and no association for lower anxiety $(\mathrm{P}=0.385)$. Classifying IDA scores into 'normal' and 'borderline/morbid' depression, and using multivariable logistic regression, VEM was associated with less depression at 7 days ( $\mathrm{OR} 2.2,95 \% \mathrm{CI} 1.3-3.7 ; \mathrm{P}=0.01)$. There were no significant differences between groups for depression or anxiety at 12 months. Conclusion: VEM may reduce depressive symptoms in stroke patients at 7 days post stroke. Reduced depression may be attributed to the exercise intervention, or the additional attention provided during VEM.

\section{Epidemiology of stroke I}

\section{FALLS IN FIRST HOSPITALIZATIONS FOR STROKE IN SCOTLAND} CONCEAL AN INCREASE IN RATES AMONGST THE YOUNG, 1986-2005 J.D. Lewsey, P.S. Jhund, M. Gillies, A. Redpath, L. Kelso, A. Finlayson, M. Walters, P. Langhorne, J. McMurray, K. MacIntyre

University of Glasgow, Glasgow, United Kingdom

Introduction: Population based studies of stroke incidence suggested that incidence was increasing until the late 1990s. Recently a number of population based studies have suggested that the incidence of stroke is now falling. It is not known if first hospitalizations for stroke have fallen in a whole country and in all age groups. Methods: Using the Scottish Linked Morbidity Record Database we identified all patients with a first episode of stroke hospitalized between 1986 and 2005 in Scotland. Rates were calculated using denominators supplied by the General Registrar Office of Scotland with interpolation of counts between census years. Rates were standardized by the direct method using the 2001 census population estimates. Poisson regression was used to model rates over time.

Results: Over the study period 162484 individuals (73676 men and 88808 women) were discharged from hospital after a first stroke. The mean age at admission did not change over the study period in men (69.2 years in $1986 / 7$ vs. 68.8 years in 2004/5) or women (73.6 years in 1986/7 vs 74.2 years in 2004/5). Age standardized rates of first hospitalization for stroke (per 100,000 population) increased in men from 157 in 1986 to 177 in 1993 before falling to 132 in 2005. In women rates of first hospitalization for stroke (per 100,000 population) increased from 180 in 1986 to 195 in 1993 before falling to 145 in 2005 . These overall falls concealed an increase in the rates amongst younger individuals. In men aged $<55$ years the relative risk (RR) of admission in 2005 vs. 1986 was 1.43 (95\% CI 1.33-1.53). Similarly, rates increased in women aged $<55$ years in 2005 vs. 1986 , RR 1.36 (1.26-1.47)

Conclusions: We report in a whole country that the rates of first hospitalizations for stroke are now falling, after increasing until the early 1990s. However, rates increased in under $55 \mathrm{~s}$. We hypothesise this trend is due to a lower threshold for recognition and investigation of focal neurological symptoms in the young.

\section{Epidemiology of stroke I}

ALL CAUSE MORTALITY RATE AFTER STROKE IN 31,821 PATIENTS WITH ATRIAL FIBRILLATION CHARACTERISED BY CHADS2 SCORES

K.M. Henriksson, B. Farahmand, S. Johansson, S. Åsberg, N. Edvardsson,

A. Terént

The Stroke Unit at Uppsala University, Mölndal, Sweden

This study examined all cause mortality rate after stroke in patients with atrial fibrillation (AF) and assessed, retrospectively, the impact by CHADS2 score. The CHADS2 score (0-6 points) is formed by assigning 1 point each for congestive heart failure (CHF), hypertension, age $>75$ years (yrs) and diabetes, and 2 points for a previous stroke. The Swedish National Quality Register for Stroke Care (RS) 2001-5 was, by records, linked to the Inpatient and Cause of Death registries. A cause-specific mortality rate after stroke by CHADS2 score and different combinations of the diagnoses in each CHADS2 score was calculated. The cohort included 31,821 (30\% of all subjects in RS) AF patients. During the mean 10 months follow-up, 11,285 deaths occurred (9,326 due to cardiovascular diseases, 4,385 to strokes). Overall mortality rate increased from $102 / 1000$ person-yrs in the lowest, to 760 for those with the highest CHADS2 score. Mortality rate varied within each CHADS2 score. The diagnoses most frequently contributing to a CHADS2 $>4$ were hypertension, age $\geq 75$ yrs and previous stroke. CHF was the most common extra fifth point whereas the influence of diabetes seems to play a lesser role. In patients with a CHADS2 score of 4 , the total and stroke mortality were approximately the same in patients with the co-morbidity CHF, age $\geq 75$ yrs and previous stroke as the combination of CHF, hypertension, age $\geq 75$ yrs and diabetes. Hypertension was not as prominent in patients with a CHADS2 score of 5 as with score 4 .

Conclusions: Total mortality rate increased more than 7-fold between subjects with the highest compared to the lowest CHADS2 score. In excess of just the numeric CHADS2 score, the combination of co-morbidity adding to the score seems to play a role.

\section{Epidemiology of stroke I}

\section{FREQUENCY, SEVERITY AND OUTCOMES OF STROKE ASSOCIATED WITH ATRIAL FIBRILLATION - THE NORTH DUBLIN POPULATION} STROKE STUDY

N. Hannon, A. Merwick, L.A. Kelly, O. Sheehan, J. Duggan, A. Moore, P.M. McCormack, E. Williams, L. Daly, P.J. Kelly

Neurovascular Clinical Science Unit, Mater University Hospital/University College Dublin, Dublin, Ireland

Prospective cohort studies in large population are important to determine the epidemiology of clinically-relevant stroke subgroups, to avoid selection bias which may complicate hospital-based studies. Although atrial fibrillation (AF) is a recognised risk factor for stroke, relatively few population-based data exist regarding the frequency, characteristics and outcome of AF-associated stroke.

The North Dublin Population Stroke Study is a prospective population-based cohort study of TIA and stroke in an urban population of 294,592 individuals over 1 year, using multiple overlapping hospital and community sources of case ascertainment and detailed clinical and follow-up assessment, according to recommended criteria for stroke epidemiology studies.

Of all 544 individuals with new stroke, AF was present in 162 (29.8\%). The crude incidence rate of AF-associated stroke was 55/100,000. While ischaemic stroke was commoner in individuals with $\mathrm{AF}(92 \%$ vs $75.9 \%, \mathrm{p}<0.001)$, haemorrhagic stroke was an important contributor to AF-associated stroke ( $8 \%$ of all). $57.8 \%$ had known AF prior to stroke onset. One-third of these were taking warfarin at stroke onset.

Compared to non-AF ischaemic stroke, individuals with $\mathrm{AF}$ were older (77.1 vs 68.7 years, $\mathrm{p}<0.001)$, had more recurrent stroke $(22.2 \%$ vs $12.8 \%, \mathrm{p}=0.01)$, greater acute severity (NIHSS 8.7 vs $6.6, \mathrm{p}<0.01$ ) and disability (RS 3.8 vs $3, \mathrm{p}<0.001$ ), and greater disability at 7 and 28 days $(\mathrm{p}<0.001)$. AF was not associated with higher 7 or 28-day case-fatality or recurrence.

$\mathrm{AF}$ was common and associated with a distinct profile of severe, recurrent and disabling stroke in older adults in our population. Targeted strategies to improve prevention of AF-stroke may have substantial benefits to population health.

\section{Epidemiology of stroke I}

COMPARISON OF INCIDENCE AND CASE-FATALITY RATES FOR STROKE AND MYOCARDIAL INFARCTION IN A FRENCH POPULATION-BASED STUDY, FROM 2001 TO 2006: THE DIJON VASCULAR (DIVA) PROJECT Y. Bejot, A. Gentil, G.V. Osseby, M. Caillier, O. Rouaud, Y. Cottin, M. Zeller, T. Moreau, M. Giroud

Dijon Stroke Registry, EA 4184, Dijon, France

Background: The burden of cardiovascular disease in developed countries is dramatic and is thus a health care priority. Nevertheless, few population-based studies have provided data to compare myocardial infarction with stroke in terms of incidence and survival.

Methods: We compared incidence and 1-month case fatality rates of stroke and myocardial infarction in a prospective well-defined population-based study, in Dijon, France (150,000 inhabitants), from 2001 to 2006

Results: Over the 5 years, we recorded 1020 first-ever strokes (485 in men and 535 in women) and 640 first-ever myocardial infarctions (403 in men, 237 in women). Mean age at vascular event onset was respectively 71.5 and 64.9 years old. Age-standardised incidence rates were 122/100,000/year for stroke and $70.2 / 100,000 / y e a r$ for myocardial infarction. For Europe as a whole, the figures were respectively 82.9 and 62.1/100,000/year. In women, the incidence of stroke was significantly higher than that of myocardial infarction, whatever the age ([RR] 2.26, 95\% CI 1.94-2.63; $\mathrm{p}<0.001$ ). In contrast,the incidence of stroke was significantly lower in men $<55$ years old ([RR] $0.60,95 \%$ CI $0.42-0.86 ; p=0.007$ ) 
and significantly higher in men $>75$ years old ([RR] 2.01, 95\% CI 1.48-2.71; $\mathrm{p}<0.001$ ). One-month case fatality rates were $9.8 \%$ for stroke and $9.84 \%$ for myocardial infarction and did not differ significantly.

Conclusion: Our study demonstrates a higher incidence of stroke compared with myocardial infarction. It provides evidence for the need to set up stroke units in France, following the model of cardiology acute care units.

\section{Epidemiology of stroke I}

\section{SECULAR TRENDS IN THE INCIDENCE OF ATRIAL FIBRILLATION} DURING 17 YEARS IN SWEDEN - INDICATIONS OF A GROWING HEALTH PROBLEM

K.M. Henriksson, N. Edvardsson, S. Johansson, N. Hammar

Laboratory Medicine Lund University Hospital, Mölndal, Sweden

Atrial Fibrillation (AF) is the most common arrhythmia and a rising incidence has been reported. This study presents recent national trends of hospital-treated $\mathrm{AF}$ in Sweden. Information on patients with AF (ICD-9 427.3 or ICD-10 148.9) from 1987-05 was acquired from the national inpatient register which covers all $\mathrm{AF}$ patients treated in hospital.Person years at risk were estimated from population register. Incidence rates were calculated by year, sex and age and age adjusted incidence rates were calculated. Overall, the age adjusted incidence of hospital-treated $\mathrm{AF}$ increased by $1.3 \%$ in men and $0.9 \%$ in women, with a substantial part occurring in the last 5 years. In relative terms this represents a more than doubled incidence as compared to the early period. This increase was less pronounced in subjects $<64$ years of age, but was consistently about twice as high in men $(0.5 \%)$ as in women $(0.25 \%)$. In subjects $65-74$ yrs the incidence of $\mathrm{AF}$ continued to be stable in women $(1 \%)$ but men showed an increasing trend from $1 \%$ at the beginning of the period to $2 \%$ towards the end. The two oldest age groups displayed a more dramatic development of AF starting in the beginning of the 1990s, the incidence in women $75-84$ years increased from 2 to $4 \%$, and in women $\geq 85$ years from 3 to $7 \%$. The increases in the corresponding age groups in men were from 2 to $5.5 \%$ and from 3 to almost $9 \%$, respectively.

Conclusions: Over the last two decades, there was a substantial increase in the incidence of hospital-treated AF in Sweden, especially in the oldest subjects and particularly marked in recent years. Possible explanations include an aging population, an improved survival in subjects with coronary heart disease and an increased awareness of the importance of detecting and treating AF.

\section{Epidemiology of stroke I}

\section{PATIENTS ENROLLED IN LANDMARK TRIALS OF ANTIPLATELET TREATMENT FOR SECONDARY PREVENTION ARE NOT} REPRESENTATIVE OF PATIENTS IN CLINICAL PRACTICE

E. Maasland, R.J. van Oostenbrugge, C. Franke, W.J.M. Scholte op Reimer, P.J. Koudstaal, D.W.J. Dippel, for the Netherlands Stroke Survey Investigators Erasmus MC University Medical Center Rotterdam, Rotterdam, The Netherlands

Background: Many randomised clinical trials (RCT) have evaluated the benefit of long-term use of antiplatelet drugs in reducing the risk of vascular events. Evidence from these trials forms the basis for many cardiovascular guidelines. Abundant and strict enrolment criteria may limit the generalisability and the applicability of results of RCTs to clinical practice.

Aim: To identify the proportion of patients with stroke or TIA in clinical practice who are eligible for participation in trials investigating antiplatelet treatment for secondary prevention.

Methods: In 11 centers in the Netherlands 972 patients with TIA or ischemic ischemic stroke were prospectively and consecutively enrolled. We applied 5 large antiplatelet trials' enrolment criteria to the patients in our survey. We distinguished between enrolment criteria aimed at selection of high-risk patients and criteria aimed at safety.

Results: In total, 886 patients were available for secondary prevention. Mean follow-up was 2.5 years. The annual rate of TIA, stroke or myocardial infarction was $6.7 \%$. The proportions of patients fulfilling the trial enrolment criteria were 25\% (MATCH), 32\% (CAPRIE), 36\% (PERFORM), 58\% (ESPRIT) and 63\% (ESPS-2). Mortality was significantly higher in ineligible patients $(27-41 \%)$ than in patients fulfilling enrolment criteria (16-20\%). Rates of vascular events were not increased in eligible patients.

Conclusions: Our data confirm that RCT enrol patients are only partially representative of patients with TIA or stroke in clinical practice. Use of less strict enrolment criteria could result in valid and more efficient selection of patients for randomised clinical trials.

\section{Brain imaging: new developments}

\section{Brain imaging: new developments}

\section{CEREBRAL AMYLOID ANGIOPATHY PREDISPOSES TO TPA RELATED HAEMORRHAGE IN PATIENTS PRESENTING WITH ACUTE ISCHAEMIC STROKE: A PIB PET STUDY}

J.V. Ly, C.C. Rowe, J.A. Zavala, H. Ma, V.L. Villemagne, G. Okeefe, U. Ackerman, J. Sachinidis, H. Tochon-Danguy, G.A. Donnan National Stroke Research Institute, Heidelberg Heights, Victoria, Australia

Background: There is increasing evidence that Cerebral Amyloid Angiopathy (CAA) may be an important predisposing factor for the haemorrhagic complications of tPA therapy in patients presenting with acute ischaemic stroke. N-methyl-[11C]2-(4'-methylaminophenyl)-6-hydroxybenzothiazole (11C PiB) is a PET amyloid ligand which also binds to beta-amyloid in cerebrovascular walls. We hypothesized that patients who developed parenchymal haemorrhage $(\mathrm{PH})$ after tPA may have increased 11C PiB PET retention compared to those with no haemorrhagic complications.

Methods: Patients treated within 3 hours of onset of ischaemic stroke with tPA were studied using PET to compare $\mathrm{PiB}$ retention in those with and withou parenchymal haematoma formation. Both were compared to age matched controls. Distribution Volume Ratio (DVR) parametric images were created using Logan graphical analysis with the cerebellar cortex as reference region. Differences between medians were assessed using Wilcoxon test.

Results: Fifteen tPA treated stroke patients (mean age 73.80 \pm SD 11) and fifteen normal controls (mean age $73.10 \pm$ SD 6) were studied. Neocortical PiB retention was significantly higher among the seven patients with PH (median DVR 1.47, IQR 1.44-1.53) compared to eight patients without PH (median DVR 1.33, IQR 1.29-1.38) ( $\mathrm{p}=0.015)$, and to normal controls (median DVR 1.31, IQR 1.20-1.39) $(\mathrm{p}=0.012)$. ROC curve analyses showed a neocortical PiB DVR of 1.41 or greater to be the optimal threshold for predicting parenchymal haemorrhage with a sensitivity of 0.86 and 1 -specificity of 0.13 .

Conclusion: Ischaemic stroke patients treated with tPA who develop PH have higher neocortical $\mathrm{PiB}$ retention compared to those without $\mathrm{PH}$, suggesting underlying CAA as a predisposing factor for tPA related haemorrhage. The high sensitivity and specificity for this finding may provide an impetus for the development of a more practical rapid pre treatment screening technique

\section{Brain imaging: new developments}

\section{IMPROVING INTER-OBSERVER AGREEMENT ABOUT BRAIN MICROBLEEDS: DEVELOPMENT OF THE BRAIN OBSERVER MICROBLEED SCALE (BOMBS)}

C. Cordonnier, G. Potter, C. Jackson, F. Doubal, C.L.M. Sudlow, J.M. Wardlaw, R. Al-Shahi Salman

Division of Clinical Neurosciences, University of Edinburgh, Lille, France

Background and purpose: Gradient echo (GRE) T2* magnetic resonance imaging (MRI) of the brains of adults with stroke frequently detects brain microbleeds (BMB). If the diagnostic and prognostic significance of BMBs are to be investigated and used for these purposes in clinical practice, observer variation in BMB assessment must be minimized.

Methods: Two doctors used a pilot rating scale to describe the number and distribution of BMBs (round, low signal lesions, $<10 \mathrm{~mm}$ diameter on GRE T2* MRI) on MRI of 264 adults with stroke or TIA, blinded to clinical data and their counterpart's ratings. Disagreements were adjudicated by a third observer, common sources of disagreement identified, and these data used to develop a revised rating scale (BOMBS), which was tested by the same observers in a separate cohort of 156 adults with stroke.

Results: In the pilot study, agreement about the presence of BMBs at any location was moderate (kappa [ $\kappa] 0.44,95 \%$ confidence interval [CI] 0.32 to 0.56 ). Agreement about the presence of $\mathrm{BMB}(\mathrm{s})$ was worse in lobar $(\kappa 0.44,95 \% \mathrm{CI} 0.30$ to 0.58 ) than in deep ( $\kappa 0.62,95 \%$ CI 0.48 to 0.76 ) or posterior fossa locations ( $\kappa 0.66$, $95 \% \mathrm{CI} 0.47$ to 0.84 ). Disagreements usually occurred in assessing whether none or one $\mathrm{BMB}$ was present and was largely due to the possible presence of BMB mimics. Assessment of BMBs using our new rating scale, BOMBS, improved agreement on the presence of $\mathrm{BMB}(\mathrm{s})$ in any location $(\kappa 0.68,95 \%$ CI 0.49 to 0.86$)$ and in lobar locations ( $\kappa 0.78,95 \%$ CI 0.60 to 0.97 ).

Conclusion: We found a variety of reasons for observer disagreement about the presence of BMBs. When these reasons were incorporated into a rating scale that also accounted for observer uncertainty, interobserver reliability improved. 


\section{Brain imaging: new developments}

RADIOLOGICAL INVESTIGATION OF NON-TRAUMATIC, SUPRATENTORIAL INTRACEREBRAL HAEMORRHAGE: TRI-NATIONAL SURVEY

C. Cordonnier, C.J.M. Klijn, J. van Beijnum, R. Al-Shahi Salman University of Edinburgh, Edinburgh, United Kingdom

Background: Knowledge about the prevalence of underlying causes of nontraumatic intracerebral haemorrhage (ICH) is limited, as is information about the diagnostic utilities of radiological investigations to detect them. EUSI guidelines do not recommend the further investigation of " $\mathrm{ICH}$ involving the putamen, globus pallidus, thalamus, internal capsule, periventricular white matter, pons or cerebellum, particularly in patients with known hypertension."

Methods: We sent a structured postal survey to assess the patterns of supratentorial ICH investigation to members of the professional organisations for stroke physicians/neurologists, neurologists, neurosurgeons, and neuroradiologists in France, the United Kingdom, and the Netherlands. We sought opinions on whether, how, and when to investigate ICH scenarios, accompanied by illustrations of brain computed tomography, in eight categories determined by ICH location (lobar versus deep), pre-stroke hypertension (present versus absent), and age ( $<45$ versus $>45$ years).

Results: We received 617 questionnaires (50\% response rate), with 99\% data completeness. Further investigation would have been undertaken by $99 \%$ of respondents in normotensive adults aged $<45$ with lobar or deep $\mathrm{ICH}, 76 \%$ of normotensive adults aged $>45$ with deep ICH, and $31 \%$ of adults aged $>45$ with deep ICH and pre-stroke hypertension. Age $<45$ years was the strongest influence on the decision to further investigate ICH (OR 16, 95\% CI 13 to 20), followed by no history of pre-stroke hypertension (OR 5, 95\% CI 4 to 6), and lobar ICH location (OR 2 , 95\% CI 1 to 2 ).

Conclusions: Youth is the strongest influence on the decision to investigate patients with supratentorial ICH. Current clinical practice does not correspond to EUSI guidelines. Studies are required to assess the diagnostic utilities of brain imaging techniques in determining ICH cause, and which patient subgroups to investigate, when, and how.

\section{Brain imaging: new developments}

COMPARISON OF PERFUSION WEIGHTED IMAGING TECHNIQUES TO ASSESS TISSUE AT RISK AND REPERFUSION IN ACUTE ISCHEMIC STROKE. A DEFUSE SUBSTUDY

V. Thijs, R. Dobosi, M. Lansberg, J. Olivot, M. Mlynash, R. Bammer, L. Wechsler, G.W. Albers

University Hospitals Leuven, Leuven, Belgium

Objective: To compare commonly used analysis methods for perfusion weighted imaging (PWI) in acute ischemic stroke patients.

Methods: PWI volumes from a prospective, multicenter study (Diffusion Weighted Imaging Evaluation for Understanding Stroke Evolution, DEFUSE) were recalculated using non arterial input function (AIF) based Time-to-peak (TTP) and Mean Transit Time (MTT) maps and compared with the original AIF-based Tmax volumes used in DEFUSE. Lesion volumes were determined by manual delineation of thresholded TTP and MTT maps with a 4 or a 6 seconds delay compared to a contralateral non-ischemic brain region. Bland-Altman plots were created and diagnostic measures of mismatch and reperfusion between MTT, TTP and Tmax were calculated. The rate of the primary endpoint of DEFUSE, favourable clinica response among mismatch patients with and without reperfusion, was recalculated based on MTT or TTP volumes.

Results: 74 patients underwent PWI and DWI within 3 to 6 hours after symptom onset and were treated with intravenous tPA regardless of the baseline magnetic resonance profile. Bland-Altman plots demonstrated large limits of agreement indicating that volumes determined by thresholded TTP or MTT differed substantially from Tmax volumes. Misclassification rates with Tmax indices of mismatch and reperfusion used as the reference standard were between $18 \%$ and $39 \%$ for mismatch and between 16 and $31 \%$ for reperfusion. If these alternative analysis techniques had been used as the primary analysis method for PWI volume determination in DEFUSE, a relationship between reperfusion and good clinical outcome in mismatch patients would not have been found.

Discussion: Commonly used analysis methods for PWI may produce misleading results regarding the presence of a mismatch or reperfusion. A relationship between good clinical response and reperfusion in mismatch patients would not have been demonstrated had thresholded MTT or TTP maps been used as the primary analysis method in the DEFUSE study.

\section{Brain imaging: new developments}

\section{OPTIMAL MISMATCH DEFINITIONS FOR DETECTING TREATMENT} RESPONSE IN ACUTE STROKE

S. Christensen, M. Parsons, D. De Silva, M. Ebinger, K. Butcher, J. Fink, S. Davis

Royal Melbourne Hospital, University of Melbourne, Australia

Background: The use of PWI/DWI (perfusion/diffusion weighted imaging) mis match (MM) may have the potential to identify patients most likely to respond to thrombolysis. Current PWI methodology has high sensitivity but poor specificity to infarct growth. This may dilute treatment effects in a penumbral selection trial due to inclusion of patients with modest probability of growth. We hypothesized that more precise MM definitions would increase the differences in growth between patients with reperfusion (RP) and without reperfusion (non-RP), with the assumption that RP is a surrogate marker for thrombolytic treatment response.

Methods: We retrospectively analyzed MRI data from the double blind randomized placebo-control EPITHET trial of tPA to identify the MM definition that best identified potential treatment responders. We included patients with acute PWI deficits, day 3-5 PWI/DWI and follow up imaging. We defined RP as PWI volume decrease of $>90 \%$ on day 3-5 and MM as PWI-DWI volume $>10 \mathrm{ml}$. The MM definition was successively tightened by increasing the Tmax threshold (the threshold used to define the ischemic penumbra) from 2 to $12 \mathrm{~s}$. For each threshold the difference in median infarct growth between RP and non-RP was assessed.

Results: Reperfusion occurred in 34 out of 81 patients. The difference in median growth between RP and non-RP was significant $(\mathrm{p}<0.001)$ at each Tmax threshold The magnitude of this difference increased as a function of the Tmax threshold (Spearmans rho $=0.94, \mathrm{p}=0.017$ ). The median growth difference between groups increased from 45 to $88 \mathrm{ml}$ when the Tmax threshold was raised from 2 to $12 \mathrm{~s}$.

Discussion: A more restrictive mismatch definition using a higher perfusion threshold is more likely to demonstrate treatment effect by selecting patients with higher probability of infarct growth. The actual selection of threshold will depend on a specificity/sensitivity trade off analysis. The details of how this optimized MM definition affects growth differences in the tPA vs. Placebo groups will be also presented.

\section{Brain imaging: new developments}

DO MR PERFUSION THRESHOLDS RELIABLY IDENTIFY CORE AND PENUMBRA OF ISCHAEMIC TISSUE IN ACUTE ISCHAEMIC STROKE? T. Carpenter, M. Lee, C.S. Rivers, J.M. Wardlaw

${ }^{1}$ University of Edinburgh, Edinburgh, ${ }^{2}$ University of Leeds, Leeds, United Kingdom

Background: MR perfusion imaging (PWI) thresholds might distinguish infarcted from at risk tissue in acute ischaemic stroke and so guide treatment decisions. We used receiver operator characteristic (ROC) curves to identify PWI thresholds in the acute stroke DWI lesion, final infarct on T2-weighted imaging and "penumbra" (tissue in the final T2 but not the DWI lesion).

Methods: Patients with acute ischaemic stroke had acute PWI, DWI, and T2 weighted imaging (FT2) at 1 month or more. We derived quantitative parametric (q) maps of cerebral blood flow (CBF), cerebral blood volume (CBV) and mean transit time (MTT). We used ROC curves to test the ability of different acute qPWI thresholds to identify tissue in the acute DWI, penumbra and FT2 lesions, per perfusion parameter and patient. We then used "figures of merit" to identify the optimum threshold, per perfusion parameter, for infarcted vs. salvaged tissue, for the whole cohort

Results: 32 patients were imaged at median 7.3 hours. Median qMTT differed between DWI (7.3s, range 3.2-14.5), penumbra $(5.96 \mathrm{~s}, 2.9-12.4)$ and FT2 $(6.5 \mathrm{~s}$, 3.0-13.4), both $\mathrm{p}<0.01$. Median $\mathrm{qCBF}$ differed between DWI and penumbra (16.5 vs $23.0 \mathrm{ml} / 100 \mathrm{~g} / \mathrm{min}$, range $6.8-58.2$ vs $11.5-71.3, \mathrm{p}=0.002)$ but not FT2 $(19.3$ 6.7-58.9, $\mathrm{p}=0.15)$. Median $\mathrm{qCBV}$ did not differ between DWI, penumbra or FT2 ROC curves and figures of merit showed that qMTT and qCBF were better than $\mathrm{qCBV}$ at identifying DWI and FT2 tissue $(\mathrm{p}<0.002)$, but because the range of PWI values in each tissue region was very wide, there was no difference in the qMTT and $\mathrm{qCBF}$ figures of merit for DWI, penumbra or FT2 lesions $(\mathrm{p}>0.222)$. Time of scan had no effect on ROC results.

Conclusions: Although median qMTT and $\mathrm{qCBF}$ values differ between tissue that survives or infarcts, there is too much heterogeneity for a single PWI threshold at any acute time point to be used to identify acute core or penumbra reliably. This indicates that PWI threshold values should not be used to guide treatment decisions. 
7 Brain imaging: new developments

INVERSE MISMATCH AND LESION GROWTH IN SMALL SUBCORTICAL ISCHEMIC STROKE

J.B. Fiebach, A. Hopt, T. Vucic, P. Brunecker, C.H. Nolte, C.A. Doege,

G.J. Jungehulsing, C. Kunze, S. Wegener, A. Villringer

Charité Universitätsmedizin Berlin, Dept of Neurology, Berlin, Germany

Background: In territorial ischemic stroke, infarction typically develops within the borders of the initial perfusion disturbance. Motivated by preliminary findings indicating a different pathophysiology in small subcortical stroke we investigated whether in these patients, lesion growth towards infarction can occur beyond the margins of the affected vascular territories of the perforating arteries.

Method: In 19 consecutive patients, stroke MRI was performed within 14 hours after ictus, and at days 2 and $6( \pm 1)$. Sizes of diffusion and perfusion lesions were determined on days 1 and 2. "Final lesion volume" was measured on T2-weighted images on day 6.

Findings: At the initial examination, the mean diffusion lesion (ADC lesion size: $1.82 \pm 1.2 \mathrm{ml})$ was larger $(\mathrm{p}=0.0002)$ than the perfusion lesion (MTT lesion size: $0.72 \pm 0.69 \mathrm{ml}$ ). Such an "Inverse Mismatch" (ADC-lesion > MTT-lesion) was present in 14/19 patients at baseline and in all patients on day 2. Final lesion volume at day 6 was $3.2 \pm 1.6 \mathrm{ml}$ which was larger than the initial perfusion deficit $(\mathrm{p}=0.02)$, a finding which was present in each patient.

Conclusion: In small subcortical ischemic stroke, "Inverse Mismatch" is frequent, and infarction develops beyond the initial perfusion lesion during the first week after symptom onset, which is strikingly different from territorial infarcts. Development of infarction in areas with initially normal perfusion indicates that cytotoxic processes probably triggered by the infarct core are a dominant mechanism for lesion growth. Areas with normal perfusion but threatened by cytotoxic damage developing over several days seem prime targets for neuroprotective therapy.

\section{Brain imaging: new developments}

PENUMBRAL MISMATCH IS UNDERESTIMATED USING STANDARD VOLUMETRIC METHODS AND THIS IS EXACERBATED WITH TIME H. Ma, J.A. Zavala, H. Teoh, J.V. Ly, T. Phan, S. Arakawa, S. Davis, L. Churilov, P. Wright, G.A. Donnan

National Stroke Research Institute, Melbourne, Victoria, Australia

Background: In acute ischemic stroke perfusion weighted image (PWI) and diffusion weighted image (DWI) mismatch on magnetic resonance imaging (MR) is used as an indicator of the presence of the penumbra and the selection of patients for clinical trials of therapy. The approximate volumetric method and the more precise co-registration method are two used for mismatch volume (MV) calculation. We hypothesized that volumetric and co-registration methods provide different estimates of the MV and its salvage rate and that these differences depend upon the time since stroke onset.

Methods: Ischemic stroke patients presenting within 48 hours of onset were imaged with MR. Volumetric MV was defined as PWI defect (Tmax 2 seconds plus) minus DWI lesion. After co-registration of images the co-registration MV was defined as the region of PWI defect which was not overlapped by the DWI lesion. The mismatch salvage rate for each method was defined as the percentage of MV not proceeding to infarction verified by repeat MR T2 sequence at about 3 months. Results: 72 patients were recruited with median age of 74.0 years. The median time to MR from stroke onset was 5.9 hours (IQR: 3.0-20.4). There was consistent underestimation of the MV using the volumetric method (median $9.3 \mathrm{ml}$, IQR: 0-63) when compared to the co-registration method (median $20.1 \mathrm{ml}$, IQR: $3.2-69.8, p<0.0001$ ). The proportion of penumbral tissue salvaged at 3 month was underestimated using the former $(32.2 \%$, IQR: 0-66.6 for the volumetric and $83.3 \%$, IQR: $66.1-98.9$ for the co-registration method, $\mathrm{p}<0.0001)$. The percentage differences in MV increased with time post stroke $(\mathrm{p}=0.006)$.

Conclusions: In acute ischemic stroke the use of a volumetric analysis of MR volumes results in a consistent underestimation of both the MV and the percentage of mismatch salvaged when compared to the more precise co-registration

Method: The degree of the former underestimation increased with time. These findings have implications for patient selection in MR based therapeutic trials using longer time windows.

\section{Brain imaging: new developments}

\section{EXPEDITING MRI-BASED PROOF OF CONCEPT STROKE TRIALS USING} AN EARLIER PRIMARY ENDPOINT

M. Ebinger, S. Christensen, D.A. DeSilva, M.W. Parsons, C.R. Levi, A. Peeters, P.A. Barber, C.F. Bladin, G.A. Donnan, S.M. Davis, for the EPITHET Investigators

Royal Melbourne Hospital, Melbourne, Parkville, Australia

Background: Prior to pivotal phase III trials of acute stroke therapies, "proof of concept' MRI trials are used to assess efficacy with surrogate imaging endpoints. We hypothesized that subacute DWI lesion volume could replace late T2-weighted lesion volume as the primary endpoint of such trials.

Methods: In the Echoplanar Imaging Thrombolytic Evaluation Trial (EPITHET), patients with acute ischemic stroke presenting within 3-6 hours, were randomised to tPA or placebo. Lesion volumes on three different time points (baseline, day 3-5, and day 90) as well as clinical scores were assessed. Only those patients who had imaging at all three time points were included in this blinded substudy.

Results: Seventy-two patients (mean age 71 years, 34 men) of 100 participants had imaging at all three time points. Median baseline DWI lesion volume was 19.6 $\mathrm{mL}$ (IQR 8.5-41.4), median DWI lesion volume on day 3-5 was $45.2 \mathrm{~mL}$ (IQR 15.9-125.1), and median T2-weighted lesion volume on day 90 was $23.0 \mathrm{~mL}$ (IQR 5.4-81.9). Correlation was moderate between baseline and day 90 lesion volumes (Pearson's rho $0.77, \mathrm{P}<0.01$ ) and strong between day 3-5 and day 90 lesion volumes (Pearson's rho 0.95, $\mathrm{P}<0.01$ ). The correlation coefficient between day 90 NIHSS score and lesion volumes was $0.64(\mathrm{P}<0.01)$ at baseline, $0.81(\mathrm{P}<0.01)$ at day 3-5 and $0.77(\mathrm{P}<0.01)$ at day 90 .

Conclusion: Subacute lesion volume correlated strongly with late lesion volume, and subacute lesion volume at day 3-5 predicted neurological deficit at day 90 These results support an early primary imaging end point for proof of concept stroke trials, expediting and economizing future research.

\section{Brain imaging: new developments}

DIFFUSION TENSOR IMAGING IN ACUTE PONTINE STROKE: REDUCTION OF FRACTIONAL ANISOTROPY OF THE PONTINE CORTICOSPINAL TRACT CORRELATES WITH A MODIFIED NIHSS

C. Ottomeyer, K. Szabo, C. Rossmanith, R. Kern, J. Binder, J.G. Hirsch, M.G. Hennerici, A. Gass

Universitaetsklinikum Mannheim, University of Heidelberg, Mannheim, Germany

Introduction: Diffusion tensor imaging (DTI) can visualize the main fiber bundles of the brain (eg, corticospinal tract, CST) and provides quantitative information on tissue integrity. We compared the DTI derived parameter fractional anisotropy (FA) of the CST of acute pontine stroke lesions with the clinical deficit

Methods: 15 acute pontine stroke patients with motor system deficits admitted to the stroke unit were studied with standardized MRI at $1.5 \mathrm{~T}$ including T2-weighted, diffusion-weighted images (DWI), and DTI (EPI, isotropic distribution along 30 directions, b-value $900 \mathrm{~s} / \mathrm{mm}^{2}, 2.5 \mathrm{~mm}^{3}$ isotropic spatial resolution, TA $7 \mathrm{~min}$ ). In each patient the CST was identified on color-coded DTI maps and FA values of the infarcted CST was compared to the contralateral non-affected CST with nordicICE software. The percentage of FA reduction was compared to the worst clinical deficit in the first 48 hours after symptom onset as measured by a modified NIHSS (motor/sensory/articulation)

Results: The mean FA of the non affected CST was 0.51, while the mean FA of the ischemic CST was 0.41 . Reduction of the FA in the affected CST ranged from 28 to $40 \%$. The mean modified NIHSS was 4.4 (SD 2.3; range 1-9). There was a significant inverse correlation of the maximal reduction of the FA value with the degree of motor paresis as evaluated by the NIHSS (beta $=-0.53, \mathrm{t}=-2.67$, $\mathrm{p}<0.05)$
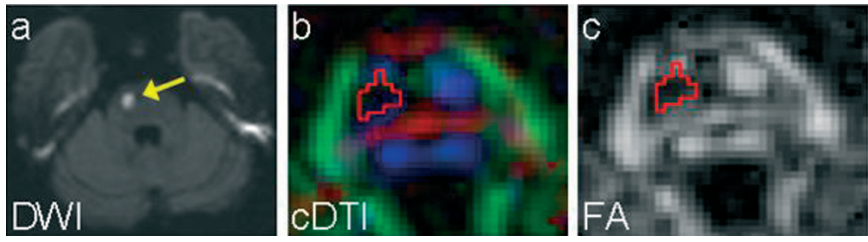

Fig. 1. Mediolateral right pontine ischemic lesion of a patient on diffusion weighted imaging (a). Colorcoded DTI shows affection of the corticospinal tract (blue fibers, red ROI) (b) and the corresponding map of the fractional anisotropy lower signal in the affected corticospinal tract (c).

Conclusions: Acute pontine stroke is a well defined anatomical and pathophysio- 


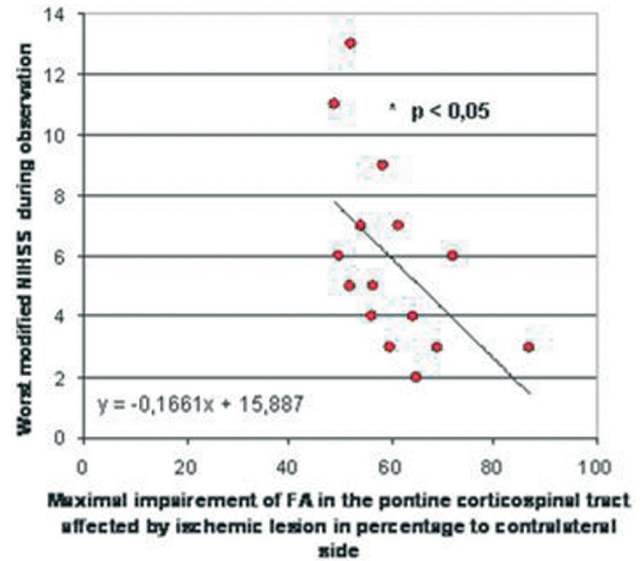

Fig. 2. Regression analysis of the worst clinical score measured in the acute phase (first $48 \mathrm{~h}$ ) in each of the 15 patients as measured by a modified NIHSS and the percentage of FA reduction in the affected corticospinal tract compared to the non-affected side.

logical model for corticospinal tract ischemia. In its acute phase the reduction of the FA of pontine corticospinal fibers correlates closely with a modified NIHSS. Whether DTI derived measures also show prognostic value in regard to the short term or long term clinical outcome will be subject of follow-up studies.

\section{Brain imaging: new developments}

PERFORMANCE OF MRI BASED CEREBRAL BLOOD FLOW MAPS IN EARLY STROKE COMPARED TO 15O-WATER POSITRON EMISSION TOMOGRAPHY: THRESHOLD ANALYSIS AND INFLUENCING FACTORS

J. Sobesky, O. Zaro Weber, W. Moeller-Hartmann, W.D. Heiss

Unversity of Cologne, Kerpenerstrasse, Germany

Background: The accuracy of perfusion weighted magnet resonance imaging (PW-MRI) based maps of cerebral blood flow (mriCBF) remains a matter of debate. We validated mriCBF on quantitative $\mathrm{CBF}$ measurement by $15 \mathrm{O}$-water positron emission tomography (petCBF) with respect to penumbral flow $(<20$ $\mathrm{ml} / 100 \mathrm{~g} / \mathrm{min}$ ).

Methods: In acute and subacute stroke patients, mriCBF and petCBF maps were compared. In a volumetric analysis, the performance of predefined mriCBF thresholds $(<40,<30,<20,<10 \mathrm{ml} / 100 \mathrm{~g} / \mathrm{min}$; quantitative analysis with arterial input function) was assessed using the volume of penumbral flow on PET as the target volume. The degree of congruence was expressed as the ratio $\mathrm{C}(\mathrm{C}=$ Volume mriCBF/Volume petCBF). The influence of vessel pathology, hypoperfusion size and time point of imaging was described.

Results: In 24 stroke patients (median time MRI to PET: 68 minutes; 16 patients imaged within 24 hours after stroke) the median volume of penumbral flow (petCBF) was $78.5 \mathrm{ccm}$. On visual inspection, an excellent qualitative congruence was found.for mriCBF. In the pooled analysis, $\mathrm{mriCBF}<20 \mathrm{ml} / 100 \mathrm{~g} / \mathrm{min}$ best identified penumbral flow (median C-ratio: 1.0) but showed a wide interindividual range (C-ratio 0.3 to 3.5 ). Ipsilateral vessel pathology, time point of imaging and size of penumbral hypoperfusion did not significantly influence the C-ratio.

Discussion: CBF maps using a threshold of $<20 \mathrm{ml} / 100 \mathrm{~g} / \mathrm{min}$ well identified penumbral flow. However, a considerable interindividual variance was found and could not be explained by routine clinical data. Our results strongly support the validity of MRI based CBF measurement in clinical routine but they also underline the need of a further specification of the MRI based mismatch concept.

\section{Brain imaging: new developments}

SENSITIVITY OF FLUID ATTENUATED INVERSION RECOVERY (FLAIR) IMAGING FOR ISCHEMIC LESIONS INCREASES WITH TIME FROM SYMPTOM ONSET TO $>90 \%$ AT THE END OF THE 6 HOUR TIME WINDOW IN ACUTE STROKE

G. Thomalla, P. Rossbach, M. Rosenkranz, S. Siemonsen, A. Krützelmann,

J. Fiehler, C. Gerloff

University Hospital Hamburg-Eppendorf, Hamburg, Germany

Background: Ischemic lesions become visible on fluid attenuated inversion recovery (FLAIR) imaging within the first hours after stroke. However, the sensitivity of
FLAIR imaging within the first 6 hours of ischemia and it's relation to time from symptom onset is not well defined. We studied the sensitivity of FLAIR imaging for acute ischemic lesions as compared to diffusion weighted imaging (DWI) during the first 6 hours of stroke.

Methods: We analyzed data of consecutive acute ischemic stroke patients studied by stroke MRI within 6 hours. MR images were rated by four experienced raters blinded to clinical information. The presence of ischemic lesions were judged in three steps: 1) on the FLAIR image blinded to DWI, 2) on the DWI, 3) on the FLAIR image with knowledge of DWI. Ischemic lesions were judged as visible if identified by at least three of four raters.

Results: Data of 104 patients (43\% females, mean age 65 y.) were analyzed. Mean (SD) time between symptom onset to imaging was $150( \pm 65)$ min, mean National Institutes of Health Stroke Scale (NIHSS) Score was $14 \pm 5$.6. Overall sensitivity of FLAIR alone was $36 \%$, compared to $97 \%$ for DWI. Knowledge of DWI information increased the visibility of ischemic lesion on FLAIR images to $63 \%$. The sensitivity of FLAIR increased over time $(<1.5 / 1.5-3 / 3-4.5 / 4.5-6 \mathrm{~h})$ for FLAIR alone $(8 \% / 31 \% / 62 \% / 44 \%, \mathrm{p}=0.009)$ and FLAIR with knowledge of DWI $(39 \% / 53 \% / 95 \% / 89 \%, \mathrm{p}<0.001)$, while sensitivity of DWI remained stable $(92 \% / 97 \% / 100 \% / 100 \%, \mathrm{p}=0.573)$. A negative FLAIR image in the case of a visible lesion on DWI allowed the allocation to $<3$ hours with a high specificity (93\%) positive predicate value $(95 \%)$.

Discussion: We demonstrate a clear time dependency of the sensitivity of FLAIR imaging for acute ischemia in hyperacute stroke. Together with information from DWI, ischemic lesions can be identified on FLAIR images in $>90 \%$ of patients after 3-6 hours. An ischemic lesion not visible on FLAIR images is very likely to be less than 3 hours old. Together with DWI, FLAIR imaging may help identify patients eligible for thrombolysis in cases of unknown symptom onset.

\section{Experimental studies I}

\section{Experimental studies I}

NUCLEAR FACTOR-KB AND POSTISCHEMIC INFLAMMATION ARE SUPPRESSED IN CD36-NULL MICE AFTER MIDDLE CEREBRAL ARTERY OCCLUSION

A. Kunz, T. Abe, K. Hochrainer, M. Shimamura, J. Anrather, G. Racchumi, P. Zhou, C. Iadecola

${ }^{1}$ Division of Neurobiology, Weill-Cornell Medical College, New York, NY, USA; ${ }^{2}$ Dept. of Neurology, University Hospital Carl Gustav Carus, Technical University Dresden, Dresden, Germany

Background: CD36, a scavenger receptor involved e.g. in inflammatory signaling (J Clin Invest 108: 785, 2001), also contributes to ischemic injury through yet unidentified mechanisms (J Neurosci 25: 2504, 2005). We investigated whether CD36 participates in the mechanisms of postischemic inflammation and thereby contributes to ischemic damage.

Methods: The middle cerebral artery was transiently occluded in wild type (WT) or CD36 null mice (KO) and 72hrs later, injury volume, mRNA expression of the nuclear factor kB (NFkB)-dependent inflammatory genes iNOS, ELAM, ICAM, and nox2, and neutrophil infiltration were analyzed. Postischemic NFkB activation was examined by electrophoretic mobility shift essay.

Results: In KO, injury volume was reduced $(-62 \pm 5 \% ; \mathrm{p}<0.05 ; \mathrm{n}=6 /$ group $)$ and inflammatory genes were markedly attenuated (iNOS: $-74 \pm 3 \%$; ELAM: $-80 \pm 11 \%$; ICAM: $-70 \pm 3 \%$; nox $2:-76 \pm 4 \% ; \mathrm{p}<0.05 ; \mathrm{n}=5$ /group). Also, the number of infiltrating neutrophils was reduced (KO: 281 \pm 93 ; WT: $1938 \pm 296 ; \mathrm{p}<0.05 ; \mathrm{n}=5$ /group). Consistent with the finding of suppressed inflammatory response in $\mathrm{KO}$, antinflammatory treatment with the iNOS inhibitor aminoguanidine reduced injury in WT $(-45 \pm 13 \%$; $n=6)$, but not in KO $(\mathrm{p}>0.05$ from vehicle; $n=6)$. In contrast, the neuroprotective agent NS398 reduced injury both in WT $(-40 \pm 15 \%)$ and KO $(-59 \pm 4 \% ; \mathrm{p}<0.05 ; \mathrm{n}=6$ /group $)$. Activation of $\mathrm{NFkB}$, a transcription factor that coordinates postischemic inflammation, was attenuated in $\mathrm{KO}$, but not in WT or in WT treated with NS398. In contrast to focal ischemia, neuroinflammation induced by intracerebroventricular injection of interleukin (IL) $1 \beta$ did not attenuate inflammatory gene expression, neutrophil infiltration or NFkB activitation in $\mathrm{KO}$ ( $>0.05 ; \mathrm{n}=5$ /group)

Discussion: The data unveil an unrecognized role of CD36 in activating NFkB and triggering postischemic inflammatory response. Moreover, CD36 is specifically involved in the mechanisms of postischemic inflammation, but not in IL1 $\beta$-induced inflammation. CD36 inhibition may be promising to counteract the deleterious effects of postischemic inflammation. 


\section{Experimental studies I}

ENDOGENOUS, ANTI-INFLAMMATORY EFFECTS OF LXR RECEPTORS IN EXPERIMENTAL STROKE

J.R. Morales, I. Ballesteros, J.G. Zarruk, F. Nombela, J. Vivancos, A. Castrillo, I. Lizasoain, O. Hurtado, M.A. Moro

Faculty of Medicine, Universidad Complutense, Madrid, Spain

Background: Recent work has identified Liver X receptors alpha (LXRalpha) and beta (LXRbeta) as potent anti-inflammatory molecules in macrophages and other immune cells. We have explored possible role and anti-inflammatory mechanisms of endogenous LXR receptors in acute stroke using LXRalpha,beta-/- mice.

Methods: Focal permanent ischemia was induced by middle cerebral artery occlusion (MCAO) in wild type controls and Nr1h3-/-Nr1h2-/- double mutant (LXRalpha,beta -/-) mice on a Sv129/C57BL/6 background, obtained through a collaboration with Drs. David Mangelsdorf and Peter Tontonoz. Infarct size was determined by 2,3,5-triphenyltetrazolium chloride (TTC) staining. Neurological assessment was performed as previously described (Caso et al. Circulation 2007). mRNA levels of inflammatory mediators were studied by quantitative RT-PCR as described (Pereira et al., JCBFM 2006).

Results: Infarct volume was greater in mice lacking both LXR isoforms when compared to control mice. Moreover, when neurological tests were applied to this sets of mice, lower scores demonstrated a protective action in WT, whereas LXRalpha,beta-/- mice showed poor neurological status. WT animals also showed a lower expression of brain mRNA levels of several inflammatory mediators than their LXRalpha, beta-/- littermates $8 \mathrm{~h}$ after MCAO.

Discussion: These data show that an endogenous LXR activatory pathway during experimental stroke mediates a potent and natural protection due to antiinflammatory mechanisms, which could be caused by physiological LXR agonists such as oxysterols. This evidence also suggests that levels of endogenous LXR agonists might serve as prognostic markers in stroke patients.

\section{Experimental studies I}

ROSIGLITAZONE INDUCES A SWITCH TO AN ANTI-INFLAMMATORY STATUS THROUGH 5-LIPOXIGENASE IN EXPERIMENTAL STROKE

M. Sobrado, M.P. Pereira, O. Hurtado, F. Nombela, E. Escolar, V.G. Romera, I. Lizasoain, J. Vivancos, M.A. Moro

Faculty of Medicine, Universidad Complutense, Madrid, Spain

Background: We have recently shown that the PPARgmma agonist rosiglitazone (RSG) induces 5-lipoxigenase (5-LO) expression when administered after experimental stroke (Pereira et al. JCBFM 2006; ISC 2007), which plays a neuroprotective effect, since inhibition of 5-LO abolishes rosiglitazone neuroprotection. Since 5-LO plays a key role in inflammatory responses, participating in the synthesis of both inflammatory and anti-inflammatory compounds, we have explored the mechanism underlying 5-LO-mediated neuroprotective after RSG administration.

Methods: Focal permanent ischemia was induced by middle cerebral artery occlusion (MCAO) in Fischer rats. Levels of the 5-LO products leukotriene B4 (LTB4) and lipoxin A4 (LXA4), as well as quantitation of PPARgamma activation were assessed by EIA following manufacturers' instructions. Infarct size was determined by 2,3,5-triphenyltetrazolium chloride (TTC) staining. RSG, T0070907 (PPARgamma antagonist) and/or LXA4 were administered 10 min after MCAO, and BWA4C (selective 5-LO inhibitor) was administered $3 \mathrm{~h}$ after experimental ischemia.

Results: RSG induced 5-LO expression, as well as the synthesis of LXA4, an effect that was inhibited by the selective 5-LO inhibitor BWA4C, indicating that RSG-induced 5-LO causes the synthesis of this eicosanoid. RSG also inhibited the synthesis of LTB4 induced by MCAO. LXA4 caused neuroprotection by reducing infarct size and neurological deficits when administered icv after MCAO. Interestingly, this effect was partly mediated by PPARgama, since it was inhibited by T0070907 co-administration.

Discussion: RSG activates a neuroprotective axis by inhibiting the synthesis of the pro-inflammatory LTB4 and promoting the synthesis of the anti-inflammatory LXA4, which exerts neuroprotection by a partly PPARgamma-dependent mechanism.
4 Experimental studies I

MAGNETIC RESONANCE MOLECULAR IMAGING OF ADHESION MOLECULE EXPRESSION IN ACUTE STROKE IN WILD-TYPE AND P-SELECTIN-DEFICIENT MICE

A.Y. Jin, J. Kaur, U.I. Tuor, D. Rushforth, J. Petersen, R.N. Muller, S. Boutry, P.A. Barber

University of Calgary, Calgary, Canada

Introduction: Endothelial activation following transient cerebral ischemia is partly mediated by the expression of the adhesion molecules P- and E-selectin. We hypothesized that magnetic resonance molecular imaging using selectin-specific Gd-DTPA-B(sLeX)A would demonstrate the differential expression of P- and E-selectin between the ischemic and contralateral hemispheres in wild-type and P-selectin-deficient mice following acute stroke.

Methods: Focal cerebral ischemia under normothermia was induced by transien occlusion of the left middle cerebral artery (i.e. $>70 \%$ decrease in cerebral blood flow for 30 minutes) in male C57 Black 6 wild-type (WT) $(n=15)$ and P-selectin knockout $(\mathrm{KO})(\mathrm{n}=11)$ mice, followed by 24 hours reperfusion and injection of contrast - either Gd-DTPA-B(sLeX)A or the non-targeted agent Gd-DTPA. MRI was done at $9.4 \mathrm{~T}$. From serial $\mathrm{T} 1 \mathrm{maps}$, the pre-post contrast difference in $\mathrm{T} 1$ in both the ischemic cortex $(\Delta \mathrm{T} 1$ Stroke side) and the control side cortex $(\Delta \mathrm{T} 1$ Control side) was evaluated at each time point. The contrast effect at each time point was evaluated as: $\Delta \mathrm{T} 1$ Stroke side - $\Delta \mathrm{T} 1 \mathrm{Control}$ side. $\mathrm{E}$ - and P-selectin were quantified by ELISA in the ischemic and control cortex and protein determination was done by modified Lowry assay.

Results: In WT mice, both Gd-DTPA-B(sLeX)A and Gd-DTPA accumulated similarly in the stroke cortex, possibly due to blood brain barrier (BBB) compromise. In $\mathrm{KO}$ mice, there was greater stroke cortex accumulation of Gd-DTPA-B(sLeX)A that was not observed with Gd-DTPA. E- and P-selectin expression in WT mice were increased preferentially in the stroke side. In $\mathrm{KO}$ mice, only E-selectin expression was increased in the stroke side.

Discussion: Interhemispheric T1 changes with Gd-DTPA-B(sLeX)A following transient focal cerebral ischemia appeared to reflect the interhemispheric differential expression of E-selectin in $\mathrm{KO}$ mice. P-selectin may play a role in BBB compromise following acute stroke. Increased expression of E-selectin in $\mathrm{P}$-selectin-deficient mice following acute stroke is a novel finding.

\section{Experimental studies I}

\section{OXYGEN THERAPY REDUCES SECONDARY HEMORRHAGE AFTER} THROMBOLYSIS IN EXPERIMENTAL THROMBOEMBOLIC STROKE L. Sun, C. Mueller, S. Heiland, C. Sommer, W. Zhou, R. Veltkamp Ruprecht-Karls-University Heidelberg, University Mainz, Heidelberg, Germany

Background: Secondary hemorrhage is an important complication of thrombolytic therapy in acute ischemic stroke. Hyperbaric oxygen (HBO) and normobaric hyperoxia (NBO) have been shown to protect the brain parenchyma as well as the brain microvascular permeability. This study investigated the effect of both oxygen therapies on hemorrhagic transformation after thrombolysis.

Methods: Wistar rats $(n=60)$ were subjected to thromboembolic middle cerebral artery occlusion (MCAO). Animals breathed either air, 100\% O2 at ambien pressure (NBO) or $100 \% \mathrm{O} 2$ at 3 bar (HBO) initiated 60 min after MCAO for $1 \mathrm{~h}$. Rt-PA $(9 \mathrm{mg} / \mathrm{kg})$ was administered intravenously to animals after oxygen therapy. Serial MR-imaging was performed $20 \mathrm{~min}, 2.5 \mathrm{~h}$ and $24 \mathrm{~h}$ after MCAO Hemoglobin concentration was quantified by spectrophotometry $24 \mathrm{~h}$ after MCAO. Bleeding was also measured semiquantitatively on trichrom stained sections and on T2* weighted MR images.

Results: Severity of ischemia did not differ among groups. HBO and NBO reduced blood-brain barrier permeability on postcontrast T1-weighted (T1w) images. Mean abnormal enhancing volumes were $68.8 \pm 44.3 \mathrm{~mm}^{3}$ in air, $43.0 \pm 23.8 \mathrm{~mm}^{3}$ in NBO and $45.6 \pm 23.6 \mathrm{~mm}^{3}$ in $\mathrm{HBO}$ at $2,5 \mathrm{~h}$; and $81.6 \pm 60.3 \mathrm{~mm}^{3}, 49.7 \pm 38.5 \mathrm{~mm}^{3}$ and $43.1 \pm 20.7 \mathrm{~mm}^{3}$ at $24 \mathrm{~h}$ after MCAO. HBO- treated rats had significantly lower hemoglobin content $(13.4 \pm 1.1 \mu \mathrm{g})$ than rats treated with $\mathrm{NBO}(15.8 \pm 2.6 \mu \mathrm{g})$ and air $(19.2 \pm 5.1 \mu \mathrm{g}, \mathrm{p}<0.05$, ANOVA). HBO and NBO treatment decreased histologic hemorrhage in the ischemic hemisphere compared to air (hemorrhagic scale: $3.4 \pm 1.5$ in $\mathrm{HBO}, 3.7 \pm 1.3$ in $\mathrm{NBO}$ und $5 \pm 1$ in air group, $\mathrm{p}<0.05$, ANOVA) Conclusions: $\mathrm{HBO}$ and NBO reduce brain microvascular permeability and hemorrhagic transformation after thrombolysis in thromboembolic cerebral ischemia. 


\section{Experimental studies I}

COMBINATION OF HYPERBARIC OXYGENATION (HBO) AND SELECTIVE JNK-INHIBITION BY D-JNK-I-1 AS A POTENT NEUROPROTECTIVE STRATEGY IN A FOCAL ISCHEMIA/REPERFUSION INJURY MODEL IN RATS

C.C. Eschenfelder, J.R. Liu, Y. Zhao, J. Meyne, G. Deuschl, A. Koch,

T. Herdegen

University Hospital Schleswig-Holstein, Campus Kiel, Germany

Background: In pervious studies, $\mathrm{HBO}$ was proven as a potent neuroprotective treatment in animal models of acute stroke by reducing apoptotic cell death Furthermore, c-Jun N-terminal kinase (JNK) is activated in ischemic neurons downstream of the activated glutamate receptors and thus mediating neuronal cell death. The highly specific peptide JKN-inhibitor D-JNK-I-1 (XG-102) has been shown to be neuroprotective in acute stroke in mammals, acting by intracellular inhibition of JNK-kinase. We thus hypothesized that combination of both treatments might enhance the neuroprotective effect of each regimen. Infarct volumetry and clinical outcome were the primary endpoints.

Methods: Male SD-rats (200-220g) were randomized in 5 groups $(n=6$, respectively). The suture model induced transient MCAO for 90 min. Any treatment started $3 \mathrm{~h}$ following MCAO. HBO was performed in a pressure chamber with $100 \%$ O2, 3 ATA for $1 \mathrm{~h}$ at $3 \mathrm{~h}$ following MCAO. D-JNK-I-1 was administered intraperitoneally $3 \mathrm{~h}$ following MCAO $(11 \mathrm{mg} / \mathrm{kg}$ ). Controls: Sham (no MCAO), MCAO alone. Treatment groups: MCAO+HBO; MCAO+D-JNK-I-1; MCAO+HBO+DJNK-I-1. Clinical outcome was used using Garcia and Bederson score. Infarct volume was assessed histologically $24 \mathrm{~h}$ following MCAO.

Results: Data are mean \pm SD. Total infact volume in controls was: Sham $(0 \pm 0) ; \mathrm{MCAO}\left(308 \mathrm{~mm}^{3} \pm 63,4\right) . \mathrm{MCAO}+\mathrm{XG}-102\left(169 \mathrm{~mm}^{3} \pm 84,6\right) ; \mathrm{MCAO}+\mathrm{HBO}$ $\left(106 \mathrm{~mm}^{3} \pm 82,4\right)$; MCAO+XG-102+HBO $\left(66 \mathrm{~mm}^{3} \pm 38,8\right)$. Garcia- and Bedersonscores showed significant improvement in all treatment groups compared to control (MCAO), however, the best results were seen in $\mathrm{MCAO}+\mathrm{XG}-102+\mathrm{HBO}$ group (12,8 vs. 7 points).

Discussion: We conclude from the presented data that combination of $\mathrm{HBO}$ and D-JNK-I-1 (XG-102) treatment in acute transient stroke in rats augments the neuroprotective effect of each therapy alone and thus might be a promising way for further treatment studies in humans.

\section{Experimental studies I}

HYPOTHERMIA OF $34^{\circ} \mathrm{C}$ PROLONGS THE THERAPEUTIC TIME WINDOW FOR THROMBOLYSIS AFTER EXPERIMENTAL THROMBOEMBOLIC STROKE

R.A. Kollmar, T. Blank, S. Schwab

University of Erlangen-Nürnberg, Erlangen, Germany

Hypothermia is neuroprotective in experimental stroke and may prolong the so far limited therapeutic time window for thrombolysis by rt-PA. Therefore, hypothermia of $34^{\circ} \mathrm{C}$ and effects on delayed thrombolysis including reperfusion-associated injury were investigated in a model of thromboembolic stroke (TE).

Methods: Male Wistar rats $(n=48)$ were subjected to TE and equally split in 4 groups: Normothermia $\left(37^{\circ} \mathrm{C}\right)$ was used for the control group $(\mathrm{C})$ and the thrombolysis group $(\mathrm{T})$ which received rt-PA $90 \mathrm{~min}$ after TE. Hypothermia $\left(34^{\circ} \mathrm{C}\right)$ was performed from 1.5 to 5 hours after TE in group $\mathrm{H}$ and in the combination group that received rt-PA in addition (HT). After 24 hours infarct size, brain edema and neuroscore were assessed. Protein markers for inflammation, adhesion and blood brain barrier (BBB) disruption were determined. MRI-measurements investigated infarct evolution and blood flow parameters.

Results: The infarct volume and brain swelling were smaller in the group $\mathrm{H}$ compared to group $\mathrm{C}, \mathrm{T}$, and HT $(\mathrm{p}<0.05$ to $\mathrm{p}<0.01)$. rt-PA treatment in group T resulted in larger infarct and brain swelling than all others. Additional hypothermic therapy in group HT reduced these parameters compared to group $\mathrm{T}(\mathrm{p}<0.05)$. Moreover, the neuroscore was better for group $\mathrm{H}$ vs. C and T. Animals of group HT performed better than in group $\mathrm{T}(\mathrm{p}<0.05)$. Lower serum concentration of sICAM-1, TIMP-1 and MMP-2 were shown for hypothermic groups $(\mathrm{H}, \mathrm{TH})$ compared to group $\mathrm{T}$.

Conclusion: Hypothermia reduced side-effects of rt-PA treatment and prolonged the therapeutic time window for thrombolysis in our model of TE.
8 Experimental studies I

INTRAVENOUS ADMINISTRATION OF 99MTC-HMPAO-LABELLED HUMAN MESENCHYMAL STEM CELLS AFTER STROKE: IN VIVO IMAGING AND BIODISTRIBUTION

O. Detante, A. Moisan, J. Dimastromatteo, M.J. Richard, L. Riou, E. Grillon, E. Barbier, M. Hommel, C. Ghezzi, C. Rémy

INSERM Grenoble Institut Neurosciences, Grenoble, France

Stem cell administration is a promising therapy for stroke. Human bone marrow mesenchymal stem cells (hMSC) offer the advantage of not originating from a tumoural source. Intravenous (IV) cell injection is more secure than a local graft after a stroke for clinical trials. No study assessed the biodistribution of IV injected hMSC after stroke. Our aim was to evaluate this distribution in rats.

Methods: Rats are allocated into 2 groups: 1) group underwent a right cerebra ischemia by middle cerebral artery occlusion (MCAo, $n=9) ; 2)$ control group $(n=9)$ without lesion. One week after MCAo, both groups received an IV injection of $3.4 \times 106$ hMSC labelled by 99mTc-HMPAO for 2 nuclear imaging $(0-2 \mathrm{~h} / 18-20 \mathrm{~h}$ after cell injection) and isolated organ counting $(2 \mathrm{~h} / 20 \mathrm{~h})$. Histology was performed. Results: By imaging, we observed a trend towards a higher activity in the brain after ischemia compared with control group. By organ counting, at $20 \mathrm{~h}$ after injection, we showed an increase of right brain activity in MCAo group $(6.5 \pm 0.9$ vs $3.6 \pm 1.2 \%$ of injected dose (ID)/g). We estimate the proportion of hMSC having migrated to the damaged brain to be 1/10,000 injected cells (2-fold higher than in control rats). In MCAo group, $2 \mathrm{~h}$ after injection, the right brain activity was higher than in the left one $(11.6 \pm 2.8$ vs $9.8 \pm 1.7 \% \mathrm{ID} / \mathrm{g})$. An initial hMSC trapping in the lungs was followed by an important decrease of pulmonary activity between 2 and $20 \mathrm{~h}$ after injection in both groups. An activity increase was showed in the spleen in contrast to the decrease in other organs. By histology, we identified hMSC in spleen, liver, lung and brain.

These results suggest that IV injected hMSC can colonize an ischemic lesion in the brain despite an initial cell entrapment in the lungs.

\section{Experimental studies I}

AUTOLOGOUS BONE MARROW ADMINISTRATION AFTER 24 HOURS REDUCES BEHAVIORAL DEFICITS AND LESION SIZE IN A NOVEL LARGE ANIMAL MODEL OF STROKE

J. Boltze, H. Barthel, A. Förschler, B. Nitzsche, C.M. Boltze, A. Reischauer, A. Hoffmann, O. Sabri, F. Emmrich, U. Gille

Fraunhofer Institute for Cell Therapy and Immunology (IZI), Leipzig, Germany

Introduction: Efficiency of experimental bone marrow (BM) cell therapies of stroke has been shown in rodents. However, transfer to clinical application requires close-to-practice large animal models. We evaluated benefit of autologous BM cell transplantation in a novel sheep model of focal cerebral ischemia allowing control of lesion size and subsequent functional deficits.

Material \& methods: 30 adult rams were subjected to permanent middle cerebral artery occlusion (MCAO) for stroke induction. 24 hours after MCAO, 15 animals received 4.0 to $5.1 \times 10 \mathrm{E} 6$ autologous mononuclear BM cells per kilogram bodyweight. 15 sheep served as controls. Functional outcome was continuously observed by behavioral phenotyping. Lesion size development was monitored by MRI and PET performed at days 1, 14 and 42 before brains were removed for further histological investigation.

Results: In BM cell treated animals, functional improvement was enhanced as compared to control animals $(\mathrm{p}<0.01)$ while control animals suffered from moderate to severe motor and sensory dysfunctions like ataxia, absent startle reflexes and spatial hemineglect for the entire observation period. MRI investigations showed similar lesion size in both groups at day $1(\mathrm{p}=0.59)$, but reduction of lesion size and hemispherical atrophy in cell treated rams 42 days upon MCAO $(\mathrm{p}<0.01)$ These findings could be confirmed by $15 \mathrm{O}$-water- and 18F-Desoxyglucose PET (p $<0.05)$. No tumor formation was observed upon BM cell administration.

Conclusion: Autologous BM marrow administration 24 hours following stroke is safe and effective in sheep. BM cell administration might be used as a novel treatment option in fighting stroke in upcoming clinical trials. 
DIFFERENTIAL MODULATION OF THE CELLULAR IMMUNE SYSTEM AFTER LARGE HEMISPHERIC VERSUS CORTICAL MURINE ISCHEMIC STROKE

A. Liesz, S. Hagmann, A. Hug, C. Zschoche, J. Adamek, T. Giese, R. Veltkamp University Heidelberg, Heidelberg, Germany

Background: Cerebral ischemia induces systemic immunodepression which increases the susceptibility to infections but may also limit autoimmune responses against the injured brain. Because infarct size may be an important determinator of the systemic immune response, we systematically compared cellular immune parameters in 2 models of focal ischemia.

Methods: In C57B1/6 mice $(\mathrm{n}=160)$, occlusion of the middle cerebral artery (MCAo) was induced either by coagulation of the distal MCA causing small cortical infarcts or by intraluminal filament for $90 \mathrm{~min}$. The respective sham operation served as control. Differential leucocyte counts were performed in blood, spleen, lymph node, and thymus. Lymphocyte subpopulations were further characterized by flow cytometry $24 \mathrm{~h}, 3 \mathrm{~d}$, and $7 \mathrm{~d}$ after MCAo.

Results: In large infarcts, a marked decrease of leucocyte counts (e.g. 58\% in lymph node) and most lymphocyte subsets was observed in all organs already $24 \mathrm{~h}$ after stroke with no differences between left- and right-sided ischemia. In contrast, small infarcts in the coagulation model led to no significant changes in differential blood count and alterations only in specific lymphocyte populations. B cells show a higher vulnerability after stroke than $\mathrm{T}$ cells. Remarkably, the regulatory $\mathrm{T}$ cell population remained stable in both models. Mature thymic cells were uneffected while immature CD4+CD8+ cells were depleted by over $90 \% 3 \mathrm{~d}$ after stroke.

Discussion: Infarct size is a major determinator of post-stroke systemic cellular immune modulation. Cerebral ischemia has a differential impact on different lymphocyte subpopulations. Whether this reflects a biologically meaningful adaptive response remains to be shown.

Supported by grants from MWK Baden-Württemberg and the Else-KrönerFresenius Stiftung

\section{Experimental studies I}

DIFFERENT INJURY MECHANISMS ARE ACTIVATED AFTER CEREBRAL ISCHEMIA DEPENDING ON THE OCCURRENCE OF REPERFUSION

M. Gutiérrez, I. Ayuso, M. Salinas, E. Díez Tejedor, J. Masjuan, M. Alonso de

Leciñana

Neurology and ${ }^{1}$ Biochemistry-Investigat Ramón y Cajal Hosp., ${ }^{2}$ Cerebrovasc.

Research, La Paz Hosp, Spain

Background and aims: Different injury mechanisms might be responsible of ischemic cerebral damage depending on the occurrence of reperfusion. We study activation of calpain, implicated in necrosis, and caspase- 3 in apoptosis, in a model of permanent or transient focal cerebral ischemia.

Materials and methods: Long- Evans rats were subjected to permanent middle cerebral artery occlusion for $5 \min (n=4), 30 \min (n=4), 1 h \quad(n=5), 3 h \quad(n=4), 72 h$ $(\mathrm{n}=6)$, to transient occlusion for $1 \mathrm{~h}$ followed by different reperfusion periods: $2 \mathrm{~h}$ $(n=3), 23 h(n=3)$ and $72 h(n=3)$, or to different duration of ischemia $1 \mathrm{~h}(n=3), 3 \mathrm{~h}$ $(n=3), 24 \mathrm{~h}(\mathrm{n}=3)$ followed by reperfusion up to 72 hours of survival. Samples were obtained from the cortex at the infarct core, the penumbra and from symmetrical areas in the contralateral hemisphere. Inmunoblots for espectrin degradation in its specific fragments of $120 \mathrm{KDa}$ by caspase and of $145 \mathrm{KDa}$ by calpain as well as cleavage of eIF4G were used to investigate activation of apoptosis and necrosis respectively.

Results: After 1 hour of permanent ischemia maximal calpain activation was observed at the infarct core while it was lower at the penumbra. There was no caspase activation. Reperfusion did not avoid calpain activation, but produced caspase activation that was greater in the core than in the penumbra.

Conclusions: Calpain activation causes necrotic death in permanent or transient focal cerebral ischemia, while caspase activation (i.e. apoptosis) is only present if reperfusion occurs. This indicates that reperfusion triggers additional injury mechanisms that should be specifically inhibited to ensure effective neuroprotection after reperfusion.

\section{Long-term outcome of stroke}

1 Long-term outcome of stroke

TWO-YEAR CARDIOVASCULAR EVENT RATES OF THE CEREBROVASCULAR DISEASE SUBPOPULATION WITHIN THE REDUCTION OF ATHEROTHROMBOSIS FOR CONTINUED HEALTH (REACH)

J. Roether, J.-L. Mas, E. Touzé, M.J. Alberts, M. Hill, G. Steg, D.L. Bhatt, P. Wilson, F. Aichner, S. Goto, On behalf of the REACH Registry Investigators Klinikum Minden, Hanover Medical School, Minden, Germany

Background: Few studies report cardiovascular (CV) event rates in "real-world" stable outpatients with stroke and/or transient ischemic attack (TIA). The two-year event rates of 18,189 cerebrovascular disease (CVD) patients within the REduction of Atherothrombosis for Continued Health (REACH) Registry are reported.

Methods: The REACH Registry is an international, prospective, observational registry of $>68,000$ patients from 44 countries, at risk of, or with established atherothrombotic disease (CVD, coronary artery disease, peripheral arterial disease). For this study we focused on 18,189 patients in the REACH Registry with CVD (stroke and/or TIA) who were followed for two years. Two-year CV event incidence rates in stroke and TIA patient populations are presented.

Results: Two-year data demonstrate high event rates of stroke (including stroke and TIA) and TIA (only) patients in this stable population (over $50 \%$ had qualifying stroke or TIA more than one year ago; Table 1). The risk of stroke, myocardial infarction (MI) and death at two years among those with stroke and TIA at entry was $11.48 \%$ for the total CVD population. Patients with a history of stroke (and TIA) are at a considerably higher risk and the incidence of major adverse cardiac events (MACE) is very high $(>20 \%)$. Event rates and predictors for events will be presented by geographic location, patient type, and physician specialty.

Table 1. Two-year event rates of CVD patients

\begin{tabular}{lccc}
\hline & Total CVD $(\mathrm{n}=18189)$ & Stroke $(\mathrm{n}=13135)$ & TIA only $(\mathrm{n}=5054)$ \\
\hline Non-fatal stroke & $5.93[5.22 ; 6.64]$ & $6.73[5.83 ; 7.61]$ & $3.93[3.26 ; 4.60]$ \\
Non-fatal MI & $2.21[1.65 ; 2.76]$ & $2.27[1.60 ; 2.94]$ & $2.04[1.30 ; 2.77]$ \\
CV death & $4.45[3.66 ; 5.22]$ & $4.78[3.77 ; 5.78]$ & $3.73[2.90 ; 4.55]$ \\
CV death/MI/stroke & $11.48[10.46 ; 12.49]$ & $12.42[11.19 ; 13.64]$ & $9.12[7.88 ; 10.34]$ \\
CV death/MI/stroke or hosp. & $21.05[20.05 ; 22.03]$ & $20.70[19.65 ; 21.74]$ & $21.94[20.51 ; 23.34]$ \\
\hline
\end{tabular}

Discussion: The risk of major secondary ischemic events, including CV death, is very high in individuals with previous stroke and/or TIA. Improved ischemic risk reduction management of CVD patients is required to prevent both morbid events and associated hospitalizations.

\section{Long-term outcome of stroke}

SIGNIFICANT VARIATION IN QUALITY OF LIFE AFTER ACUTE ISCHAEMIC STROKE BETWEEN WESTERN COUNTRIES: DATA FROM THE 'TINZAPARIN IN ACUTE ISCHAEMIC STROKE TRIAL' (TAIST) L.J. Gray, N. Sprigg, P.M.W. Bath, on behalf of the TAIST Investigators Institute of Neuroscience, University of Nottingham, Nottingham, United Kingdom

Background: Multiple studies have reported that functional outcome (death or dependency) varies significantly between countries. However, differences in quality of life (QoL) after stroke by country have yet to be reported.

Methods: TAIST was a randomised controlled trial assessing the safety and efficacy of tinzaparin versus aspirin in patients with acute ischaemic stroke across 11 countries. Counties were grouped into 5 geographical regions - British Isles (Ireland, UK), Franco (Belgium, France), North America (Canada), North-West Europe (Germany, Netherlands), and Scandinavia (Denmark, Finland, Norway, Sweden). QoL was measured at 180 days post randomisation using the short-form 36 health survey, which assesses QoL across eight physical and mental domains. The relationship between region and each domain was assessed using ordinal regression adjusted for treatment assignment, case mix and service quality relative to the British Isles.

Results: 1,220 survivors were included in this analysis. Significant differences in QoL were present between the countries: North West Europe rated their QoL highest in terms of physical functioning, physical role, bodily pain and vitality in comparison to the other regions. Relative to the British Isles QoL for the physical domain was $77 \%$ higher in Franco countries, and $400 \%$ higher in North West Europe; similarly social functioning was $75 \%$ higher in Franco countries and $340 \%$ higher in Scandinavia. Franco countries scored significantly lower for emotional role and mental health compared to the British Isles (Odds Ratio (OR) 
0.49 95\% confidence interval (CI) $0.26-0.92$, and OR $0.4195 \%$ CI $0.23-0.72$ respectively).

Conclusions: QoL varies considerably between countries, even when adjusted for prognostic case mix variables and measures of care quality. Differing health care systems, and the management of patients with acute stroke, may contribute to these findings.

\section{Long-term outcome of stroke}

MANAGEMENT AND OUTCOME OF ACUTE STROKE, A STUDY OF 105,043 PATIENTS REPORTED TO THE SWEDISH NATIONAL QUALITY REGISTER FOR STROKE CARE (RIKS-STROKE)

A. Terént, S. Åsberg, K. Henriksson, B. Farahmand, K. Asplund, B. Norrving, B. Stegmayr, P.O. Wester, K. Hulter Åsberg

The Stroke unit at Uppsala University Hospital, Uppsala, Sweden

Background: Stroke unit care has proven superior to care at general wards in randomised controlled trials (RCTs). The number of hospital beds has been reduced, and today stroke patients are admitted to stroke units (SU), general wards (GW), observational units (OU), intensive care units (ICU) and other type of wards (OW). We have compared the long-term survival of stroke patients treated at different ward types in Sweden.

Methods: Stroke patients who were registered in the Swedish National Quality Register for Stroke Care (Riks-Stroke), from January 1st 2001 through December 31st 2005 were followed until January 31st 2007 by linking Riks-Stroke with the Causes of Death Register. Kaplan-Meier analyses were used to estimate survival times and Cox regression to adjust for different case mix.

Results: Sixty per cent of the patients were admitted to SUs, $22.7 \%$ to GWs, $11.2 \%$ to OUs, $3.7 \%$ to ICUs and $2.4 \%$ to OWs. The mean survival time in days (95\% CI) was 1432 (1425-1441) in SUs, 1252 (1239-1264) in GWs, 1349 (1331$1367)$ in OUs, 1222 (1188-1256) in ICUs and 1435 (1396-1473) in OWs. These relationships persisted after adjustment for age, sex, type of stroke (intracerebral haemorrhage, cerebral infarction) and living conditions. The inclusion of other variables, i.e. level of consciousness, vascular risk factors and medication, does not change outcome data.

Discussion: Stroke unit care appears to be associated with better long-term survival than care at other types of wards. The greatest difference was found between Sus and GWs; this is in line with RCTs and previous observations in Riks-Stroke. The difference between Sus and ICUs is not conclusive in the present study and needs to be further explored.

\section{Long-term outcome of stroke}

\section{PATIENT AND CARER EXPERIENCES OF COMMUNITY-BASED STROKE CARE IN IRELAND: THE IRISH NATIONAL AUDIT OF STROKE CARE (INASC)}

A. Hickey, F. Horgan, H. McGee, D. O'Neill, on behalf of the INASC team Royal College of Surgeons in Ireland, Dublin, Ireland

Background: The high level of physical and psychological morbidity following stroke is associated with significant burden for patients and carers. An important input to improving quality of stroke care is to ask people with stroke about their experiences. This study sought to document patient and carer experiences of stroke services and provides first evidence on the status of community-based stroke service provision in Ireland.

Methods: Stroke patients discharged from four nationally representative hospitals in Ireland, stratified by age $(+65)$, sex and time since stroke (6-12 months or 24-36 months), and their carers, were selected. Quality of care was assessed with questions from the UK Healthcare Commission National Patient Survey (2005), alongside standardised measures of functional ability (Barthel Index), depression (HADS) and vulnerability (Vulnerable Elders Survey). Similar measures were used with the carer group.

Results: 139 patients (55\% response) and 72 carers (80\% female; 69\% response) participated. Almost half of patients $(47 \%)$ had travelled to hospital using private transport at the time of stroke. No patients had been managed in hospital in a stroke unit. A majority of patients reported poor communication at the time of discharge from hospital, $67 \%$ not receiving a designated contact name. Community rehabiliation was not available in some cases, particularly for some disciplines (e.g., psychological and speech and language services). $30 \%$ of patients scored in the depressed range and $57 \%$ were classified as vulnerable, significantly moreso for women $\left(\chi^{2}=5.93, p<0.05\right) .15 \%$ of carers scored in the depressed range, with $11 \%$ classified as vulnerable.

Discussion: Overall, patients and carers reported variable experiences of community stroke care in Ireland. While a majority were satisfied with hospital based care, many were unable to obtain needed rehabilitation and support services once discharged home. Given that a majority of patients with stroke are discharged to the community, optimisation of community-based stroke care is central to minimising disability and maximising quality of life after stroke.

\section{Long-term outcome of stroke}

\section{SEX DIFFERENCES IN SURVIVAL AND FUNCTIONAL OUTCOME AFTER STROKE - THE SOUTH LONDON STROKE REGISTER}

M.A. Busch, P.U. Heuschmann, Ö. Saka, C.D.A. Wolfe

King's College London, London, United Kingdom

Background: Recent studies suggest that women may have poorer stroke outcome but data with regard to specific outcomes are conflicting. We investigated sex differences in stroke outcome and examined the contributions of other patient characteristics using a hierarchical conceptual framework.

Methods: Data were collected from the South London Stroke Register, a population-based register of first-ever stroke in London, UK. All registered patients were prospectively followed up annually for up to 10 years. Sex differences in survival and dependence (Barthel Index <20) at 1 year were assessed by hierarchical Cox regression and logistic regression analyses, adjusted for sociodemographics, comorbidities, stroke severity and service use.

Results: Of 2874 patients with stroke in 1995-2004, 1447 (50.4\%) were women (mean age $73.8 y$ vs $67.4 y$ in men). In survival analysis, women had a $42 \%$ higher risk of death (hazard ratio (HR) $1.42 ; 95 \%$ CI 1.29-1.56) and half of this effect was mediated through age (age-adjusted HR 1.20; 95\% CI 1.09-1.32). The effect of sex remained almost unchanged after additional adjustment for sociodemographics and comorbidities, but was no longer significant after further adjusting for stroke severity. Surviving women were more often dependent at 1 year than men (OR 1.76; 95\% CI 1.42-2.17). This effect of sex decreased after adjusting for age (age-adjusted OR 1.58; 95\% CI 1.27-1.98), was not further mediated through sociodemographics or comorbidities, but again partly mediated through stroke severity (OR 1.40; $95 \%$ CI 1.06-1.85).

Conclusion: The poorer survival of women after stroke observed in this study can mainly be attributed to higher age and stroke severity, while their poorer functional outcome cannot be fully explained by the included variables.

\section{Long-term outcome of stroke}

\section{LONG-TERM FOLLOW-UP AFTER FIRST-EVER STROKE AMONG THE BESANÇON REGISTRY: MORTALITY AND RECURRENCE OF VASCULAR EVENTS \\ P. Decavel, E. Medeiros, E. Revenco, T. Moulin \\ University Hospital, Besancon, Besancon, France}

Background: Regional healthcare networks have been established to optimise care provision in France. The objective of this study was to evaluate the impact of implementaion of a regional stroke unit on long term outcome in terms of mortality and recurrence after eighteen months after a first stroke.

Method: We compared three different periods (1987-1994: first period; 1998-2002: second period; 2003-2006: third period) corresponding respectively to unstructured stroke care, the period in which a structured hospial stroke unit was set up and the period in which the regional network was established. During the three periods, all patients presenting a first-ever stroke (infarction, haematoma or transient ischaemic attack - TIA) hospitalised in the University Hospital in Besançon were prospectively entered into a patient registry and evaluated using standard diagnostic criteria. Outcome was analysed during the first 18 months post-stroke and adjusted according to age, sex and stroke sub-types.

Results: We analysed 6877 patients, whose mean age was 73 years and of whom $55 \%$ were men. Of these, 4770 presented an infarction, 765 a haematoma and 1295 a TIA. The cohorts differed betwen the three periods in terms of gender $(p=0.596)$ and stroke subtypes $(\mathrm{p}<0.001)$. The mortality rate at one month after stroke for the three periods was $15.9 \%, 11.7 \%$ and $6.6 \%(\mathrm{p}=0.000)$ respectively. Eighteen months after stroke, the long-term survival rate was $87 \%, 90 \%$ and $94 \%$. The rate of recurrence of vascular events could only be assessed for the last two periods: $4.5 \%$ and $2.60 \%$

Conclusion: This study suggests that patient management within structured regional stroke networks has a positive impact both on immediate mortality and on long-term survival in Besançon. 
7 Long-term outcome of stroke

ALBUMIN-CORRECTED SERUM CALCIUM CONCENTRATIONS AND LONG-TERM MORTALITY IN PATIENTS WITH ACUTE STROKE

R. Schwartz, S. Apel, Y. Schwammenthal, O. Merzeliak, R. Tsabari, D. Orion,

D. Tanne

Sheba Medical Center and Tel Aviv University, Tel Hashomer, Israel

Background: Calcium may influence the molecular pathways of neuronal death. Our aim was to examine the association between serum calcium and albumincorrected calcium levels and stroke outcome in a large prospective cohort of unselected patients with acute stroke.

Methods: In a prospective cohort study, consecutive patients hospitalized due to acute stroke (ischemic or intracerebral hemorrhage) throughout a large medical center were systematically assessed and followed for 1-year. Baseline total and albumin-corrected serum calcium levels were collapsed into quintiles and associations with stroke severity and outcomes examined. ORs (95\% CI) adjusted for age, gender, stroke type, stroke severity, anemia, chronic kidney disease, malignancy, chronic heart failure, and prior disability were estimated.

Results: Among 785 patients (mean age $71 \pm 13$ yrs, 58\% males) rates of severe stroke (baseline NIHSS $>10$ ) by quintiles of albumin-corrected calcium levels were: Q-I (<8.84) 33\%, Q-II (8.84-9.13) 26\%, Q-III (9.14-9.31) 29\%, Q-IV (9.32-9.61) $22 \%$, and $\mathrm{Q}-\mathrm{V}(>9.61 \mathrm{mg} / \mathrm{dL}) 32 \% ; \mathrm{p}=0.008$. After adjustment for differences in baseline characteristics, a U-shaped relationship between albumin-corrected calcium levels and mortality was observed. Using Q-IV as the reference, the adjusted ORs for 1-months mortality were: for Q-I 3.38 (1.38-8.31), Q-II 1.38 (0.51-3.76), Q-III $1.16(0.44-3.08)$ and Q-V $1.58(0.63-3.95)$. The adjusted ORs for 1-year mortality were: for Q-I 2.08 (0.96-4.58), Q-II 1.38 (0.61-3.14), Q-III 1.21 $(0.52-2.79)$ and Q-V $2.55(1.22-5.47)$. No significant associations were observed with long-term disability (Barthel Index $<75$ ).

Discussion: Serum calcium concentrations are early markers of stroke severity and outcome in acute stroke. Findings suggest a U-shaped association between albumin-corrected calcium concentrations and long-term mortality after acute stroke.

\section{Long-term outcome of stroke}

LONG-TERM FOLLOW-UP AFTER SUBOCCIPITAL DECOMPRESSIVE CRANIECTOMY FOR MALIGNANT CEREBELLAR INFARCTION

T. Pfefferkorn, U. Eppinger, J. Linn, A. Straube, T. Birnbaum, M. Dichgans,

S. Grau

Klinkum Grosshadern, University of Munich, Munich, Germany

Background and purpose: Suboccipital decompressive craniectomy is a lifesaving intervention for patients with malignant cerebellar infarction. However, long-term outcome has not been systematically analyzed.

Patients and methods: In this monocentric retrospective study we reviewed the charts of all consecutive patients that were treated by suboccipital decompressive craniectomy for malignant cerebellar infarction in our institution between 1996 and 2006. Prior to surgery, all patients presented with space-occupying cerebellar infarction and a declining level of consciousness. Outcome data in survivors were obtained by telephone (modified Rankin Scale) and SF-36 questionnaires (quality of life).

Results: A total of 57 patients were identified. The mean age was 59.2 years (27-81 years). Five patients were lost for follow-up. In the remaining 52 patients, the mean follow-up interval was 4.3 years (1-11 years). Twenty-one patients (40.4\%) had died, $15(29 \%)$ within the first six months after surgery. Among the 31 long-term survivors, functional outcome was good in 26 (mRS of 0-3: 84\%) and poor in five patients (mRS of 4-5: 16\%). All but two patients (94\%) expressed retrospective contentment with the surgical intervention. The data on quality of life are pending and will be presented at the conference.

Conclusions: The majority of survivors after suboccipital decompressive craniectomy for malignant cerebellar infarction have a good long-term functional outcome. Retrospectively, almost all of them are content with having received this treatment.

\section{Long-term outcome of stroke}

POSTTRAUMATIC STRESS DISORDER EXPLAINS REDUCED QUALITY OF LIFE IN SAH PATIENTS IN BOTH THE SHORT AND LONG-TERM

A.J. Noble, T. Schenk

Durham University, United Kingdom

Background: Subarachnoid haemorrhage (SAH) frequently has a drastic long-term impact on patients' health-related quality of life (QoL). Neurological problems alone cannot explain the reduced QoL. Here we examine whether posttraumatic stress disorder (PTSD) and fatigue can provide a satisfactory explanation for this reduced QoL in both the short- and long-term.

Methods: We prospectively studied a representative sample of 105 patients with spontaneous, non-traumatic SAH. Patients were examined twice, firstly at 3 months post-ictus and again at 13 months post-ictus. Examinations included assessments of PTSD, fatigue, sleep, cognitive and physical outcome and QoL. In addition, patients' coping skills were assessed. Regression analyses were performed to identify the best predictors for the patients' QoL and PTSD.

Results: $37 \%$ of patients met the diagnostic criteria for PTSD at both assessment points. This is a staggering four-fold increase when compared to the incidence rate in the general population. Fatigue in SAH patients was also consistently higher than in matched controls. PTSD was the best predictor for mental $\mathrm{QoL}-$ the domain most persistently impaired - in both the short- and long-term. It also helped predict physical QoL levels reported more than one year post-haemorrhage. Moreover, PTSD was consistently linked to increased levels of sleep problems and therefore to increased levels of fatigue. To establish the cause of PTSD a logistic regression was performed. This showed that maladaptive coping was the best predictor of PTSD.

Discussion: PTSD explains why SAH patients with relatively good clinical outcome, nevertheless have persistently reduced QoL. PTSD also explains to a great extent patients' chronic fatigue. Patients' levels of fatigue were at times in fact, even higher than in cancer patients undergoing chemotherapy. The findings suggest that since poor coping skills seem to be the main cause of PTSD, teaching patients better coping skills early on might prevent PTSD, fatigue and the resulting drop in QoL.

\section{Acute stroke: treatment concepts III}

\section{Acute stroke: treatment concepts III}

BP LOWERING IN ACUTE STROKE - THE CHHIPS PILOT STUDY

A.K. Mistri, T.G. Robinson, J.F. Potter, CHHIPS and COSSACS Trial Groups University of Leicester/University of East Anglia, Leicester, United Kingdom

Introduction: Elevated blood pressure (BP) levels immediately post-stroke are associated with an adverse prognosis in terms of death and dependency. However the benefit of lowering these elevated levels is still unclear. The CHHIPS trial tested the BP lowering efficacy of lisinopril and labetalol in acute stroke patients.

Methods: This multi-centre, prospective, randomised, double-blind, placebocontrolled, titrated-dose trial, recruited hypertensive cerebral infarct and haemorrhage patients (systolic BP $>160 \mathrm{mmHg}$ ) within 36 hours of symptom onset. Target systolic BP was $145-155 \mathrm{mmHg}$ or a $15 \mathrm{mmHg}$ SBP reduction within the first 24 hours achieved by up-titration of labetalol (lab) or lisinopril (lis) or placebo (plac), established treatment being continued for 2 weeks.

Results: 179 patients were recruited with a baseline BP $182( \pm 17) / 95( \pm 13 \mathrm{mmHg}$. $\mathrm{BP}$ fell from randomisation to 24 hours in all groups (mean (SE): lab 18(3); lis 25(3); plac 11(3) $\mathrm{mmHg}$ ) and remained lower than baseline at 2 weeks (lab 11(2); lis 15(2); plac 9(2) $\mathrm{mmHg}$ ). Over the initial 24 hours, SBP reduction was significantly greater in the lisinopril group $(\mathrm{p}=0.001)$ and the combined active treatment group $(\mathrm{p}=0.004)$, but not in the labetalol group $(\mathrm{p}=0.096)$, compared to placebo (lab 31(3); lis 32(3); combined lab/lis 31(2); plac 24(3) $\mathrm{mmHg}$ ). At 2 weeks, SBP reduction was significantly greater in the combined active treatment group compared to placebo ( 31 (2) vs 24 (3) $\mathrm{mmHg}, \mathrm{p}=0.045$ ), with no significant difference in SBP or DBP reduction at 2 weeks for either treatment agent compared to placebo. There were no significantly greater adverse events with active treatment. Conclusions: Lisinopril produces sustained SBP reduction at 24 hours. The hypotensive effects of labetalol are short-lived and frequent dosing/infusion may be required to produce sustained BP reduction. Further dose escalations are likely to be required for sustained BP reduction beyond 24 hours.

\section{Acute stroke: treatment concepts III}

LOWERING BLOOD PRESSURE IN DYSPHAGIC ACUTE STROKE PATIENTS - CHHIPS PILOT STUDY

A.K. Mistri, T.G. Robinson, J.F. Potter, CHHIPS Trial Group

University of Leicester, Leicester, United Kingdom

Introduction: Although elevated systolic blood pressure (SBP) levels immediately post-stroke are associated with an adverse prognosis in terms of death and dependency, the benefits of reducing these raised levels is still unclear. To date dysphagic stroke patients have in general been excluded from BP lowering trials. 
The CHHIPS trial tested the BP lowering efficacy of sublingual lisinopril and intravenous labetalol in acute stroke patients with dysphagia.

Methods: This multi-centre, prospective, randomised, double-blind, placebocontrolled, titrated-dose trial, recruited hypertensive $(\mathrm{SBP}>160 \mathrm{mmHg}$ ) cerebral infarct and haemorrhage patients within 36 hours of symptom onset. Target SBP was $145-155 \mathrm{mmHg}$ or a $15 \mathrm{mmHg}$ SBP reduction within the first 24 hours achieved by up-titration of intravenous labetalol, sublingual lisinopril or placebo.

Results: 83 patients with dysphagia were recruited over 36 months. Repeated measures analysis for SBP for the three treatment arms at 4, 8 and 24 hours showed an overall significant difference between treatments with time $(\mathrm{p}<0.0001)$. There was a borderline significant reduction in SBP in the lisinopril dysphagic group compared to placebo at 8 hours (mean reduction $10 \mathrm{mmHg},-1$ to $21, \mathrm{p}=0.07$ ) and a significant reduction at 24 hours (mean reduction $12 \mathrm{mmHg}, 2$ to $23, \mathrm{p}=0.024$ ), but not at 4 hours. In contrast, the labetalol dysphagic group had a significant reduction in SBP compared to placebo at 4 hours (mean reduction $16 \mathrm{mmHg}, 95 \%$ CI 26 to $5, \mathrm{p}=0.005$ ), but not at 8 and 24 hours. No increase in serious adverse events was seen with active treatment.

Conclusions: Sublingual lisinopril is an effective antihypertensive agent in early acute stroke, with a sustained BP lowering effect at 24 hours. Intravenous labetalol is efficacious over the short-term (4 hours), and repeated dosing/infusion may be required to produce sustained BP reduction. We have demonstrated BP lowering efficacy of lisinopril administered via a novel (sublingual) route. Both active treatments appear to have an acceptable safety profile.

\section{Acute stroke: treatment concepts III}

\section{CANDESARTAN GRADUALLY LOWERS BP FOLLOWING ACUTE ISCHAEMIC STROKE: THE ACUTE CANDESARTAN CILEXETIL OUTCOMES STROKE TRIAL (ACCOST)}

R.E. O’Brien, L. Palmer, D. Johnston, A.J. Hildreth, J.E. O'Connell, C.S. Gray University of Newcastle upon Tyne, Sunderland, United Kingdom

Background and aims: Hypertension is an important modifiable risk factor for first ever and recurrent stroke. Evidence exists to support the active lowering of BP from several weeks after stroke, even from 'normal' levels. Early introduction of the angiotensin receptor blocker Candesartan in acute stroke patients with severe hypertension reduces vascular events without lowering blood pressure. ACCOST examined the safety of Candesartan given within 72 hours of stroke in normotensive and mildly hypertensive patients.

Methods: Two-phase randomised placebo controlled trial. Phase 1: Four-week double blind comparison of candesartan $4 \mathrm{mg}$ versus placebo, increased to $8 \mathrm{mg}$ if titration criteria were met, without target BP. Phase 2: Eight-week open-label study of candesartan versus an angiotensin converting enzyme-inhibitor (perindopril) based regime. Eligible patients were within 72 hours of acute ischaemic stroke, with $\mathrm{BP}>120 / 70$.

Results: Thirty-eight patients were included in the study, 19 in each group. Mean BP pre-treatment was $147 / 84 \mathrm{mmHg}$ in the candesartan group and $148 / 90 \mathrm{mmHg}$ in the placebo group. Results of phase 2 are reported here. After 12 weeks, mean ( $95 \% \mathrm{CI})$ systolic BP changes were $-10.0(-21.2$ to 2.7$) \mathrm{mmHg}$ for candesartan and $-1.6(-10.5$ to 7.3$) \mathrm{mmHg}$ for placebo/perindopril $(\mathrm{p}=0.223)$. Mean $(95 \% \mathrm{CI})$ diastolic BP changes were -3.7 (-10.2 to 2.7) $\mathrm{mmHg}$ for candesartan and $-1.9(-9.3$ to 5.5$) \mathrm{mmHg}$ for placebo $(\mathrm{p}=0.699)$. Precipitous falls in BP were not observed. More patients in the candesartan treated group were at target BP by 12 weeks $(42 \%$ vs. $26 \%$ ).

Conclusions: Mean BP changes over 12 weeks were not significantly different between the groups although a trend towards greater BP reduction was observed with candesartan. Early introduction of candesartan appears to be safe and is more likely to lead to early achievement of target BP. Further work is required to investigate whether early use of candesartan influences outcome after acute ischaemic stroke.

\section{Acute stroke: treatment concepts III}

FEASIBILITY AND TIME EFFICIENCY OF MRI BEFORE THROMBOLYTIC TREATMENT OF ACUTE STROKE IN THE 3 HOUR TIME WINDOW

C. Sølling, M. Ashkanian, N. Hjort, G. Andersen, L. Østergaard

Center of Functionally Integrative Neuroscience, Aarhus, Denmark

Background: Magnetic resonance imaging (MRI) in acute stroke patients provides important diagnostic information and may facilitate individualized treatment, ye longer scan times could delay treatment. This study estimates feasibility of MRI, and analyzes time data in an acute stroke referral center.

Materials and methods: MRI was first choice imaging modality as part of a project in normal working hours. We prospectively recorded consecutive patients referred as candidates for thrombolytic treatment in a two year time period. Contraindications to MRI and reasons for not performing MRI were noted. Time of onset of symptoms, arrival at the hospital, imaging time and time to treatment were carefully recorded and important time intervals were calculated.

Results: We received 174 patients of which 161 required acute imaging. MRI was performed in 141 , yielding a feasibility rate of $88 \%$. With an experimental MRI protocol, median "door-to-needle time" (DNT) in MR scanned patients (70 minutes), did not differ from DNT of patients treated after conventional CT during the same time period at our institution outside the project $(\mathrm{N}=17, \mathrm{DNT}=66$ minutes, $\mathrm{p}=0.27$ ) or the SITS-MOST registry (DNT $=68$ minutes)

Conclusions: MRI can be performed in the majority of acute stroke patients without delaying treatment.

\section{Acute stroke: treatment concepts III}

\section{STATIN TREATMENT PRIOR TO CAROTID ENDARTERECTOMY (CEA)} REDUCES INFLAMMATION AS SEEN ON CONSECUTIVE 18FDG-PET IMAGING

M.A. Font Padros, A. Fernandez, I. Rico, M.M. Turu, A. Luque, C. Gamez, M. Slevin, F. Rubio, L. Badimon, J. Krupinski

Hospital Universitari de Bellvitge, L’Hospitalet de Llobregat (Barcelona), Spain

Background: Vulnerable atherosclerotic plaques have been characterized by their expression of high numbers of inflammatory cells. Fluorine-18 fluorodeoxyglucose (18-FDG) accumulates in inflamed tissues and several groups have established that inflamed blood vessels and atherosclerotic lesions have increased uptake of 18-FDG. Our aim was to investigate the effect of statin treatment prior to CEA on 18-FDG carotid uptake on consecutive PET scans in the contralateral carotid artery with low to moderate stenosis.

Methods: We enrolled 14 patients with unilateral carotid artery stenosis scheduled for CEA. In group A $(n=7)$ patients received statins for at least 4 weeks prior to the first PET and continued throughout the study. In a group B $(n=7)$ patients did not receive treatment with statins. The follow-up 18-FDG-PET was performed $116 \pm 22$ days after CEA. A semiquantitative analysis of FDG-uptake values based on maximum standardized uptake value. Slice-activity curves were calculated in order to visualize plaque and basal metabolism. Carotid plaque morphology was assessed by examination of haematoxylin and eosin stained sections and the peroxidase method was used for immunohistochemical staining with monoclonal anti-CD68 antibody. Computer-assisted planimetry was used to quantify areas of staining in order to validate the PET FDG uptake correspondence with macrophage infiltrates.

Results: Statin treatment prior to the CEA (group A vs. B) was associated with a more pronounced decrease in 18-FDG-uptake on second PET in the contralateral carotid artery $(\mathrm{p}<0.05)$. This effect was independent of levels of total-cholesterol, LDL-cholesterol, HDL-cholesterol or triglycerides between two studied groups. We found a statistically significant correlation between the degree of FDG accumulation and the presence of macrophages in the atherosclerotic plaques $(\mathrm{R}=0,8, \mathrm{p}<0,005)$.

Discussion: This pilot study demonstrates that pleiotrophic effects of statins in reduction of inflammation can be visualized in vivo by 18 -FDG-PET imaging.

\section{Acute stroke: treatment concepts III}

\section{SYSTEMATIC REVIEW OF BLOOD BIOMARKERS TO AID DIAGNOSIS IN} ACUTE ISCHAEMIC STROKE

W.N. Whiteley, M.C. Tseng, P. Sandercock

University of Edinburgh, Edinburgh, United Kingdom

Introduction: Blood markers could help improve the diagnosis of stroke in the acute setting, especially if imaging is normal or not immediately available. We planned systematically to review the diagnostic performance of blood biomarkers. Methods: We searched Medline and EMBASE from 1966 to 1st December 2007, reference lists and personal files. Two authors reviewed the papers and performed data extraction.

Results: We identified 3101 studies of which 21 were eligible. Diagnostic test performance was formally examined in only $4 / 21$ studies. Sensitivity and sensitivity, applying a prespecified diagnostic threshold for a positive test, was examined in $6 / 21$ studies. The remaining 15 studies that employed a data-dependent (post hoc) threshold, $0 / 15$ validated the test in a new cohort. 4/21 studies measured the biomarker blinded to clinical status. The 21 studies tested 58 single biomarkers and 7 panels (made up of several markers) in 2928 stroke patients and 1569 controls. Sensitivity was $>90 \%$ for: nucleoside diphosphokinase A (NDKA), RNA binding protein, UFD-1, NMDA receptor 2 fragment, NMDA receptor antibodies. Specificity was over $90 \%$ for: RNA-binding protein, ubiquitin fusion degradation protein -1, NDKA, glutathiosone-s-transferase P, ischaemia modified albumin, 
visin like protein, beta globin DANN, NR2 fragments, S100 beta, fatty acid binding protein, neurone specific enololase, NR2A/2B Ab, myelin basic protein and thrombomodulin. Choice of control subject had a substantial effect on test specificity,

Conclusions: Several blood markers performed well but need to be validated in new cohorts of patients with suspected stroke, with blinding and comparison with appropriate controls. None can yet be recommended for use in routine clinical practice.

\section{Acute stroke: treatment concepts III}

\section{HYPERGLYCEMIA AND RESISTANCE TO EARLY AND LATE MIDDLE CEREBRAL ARTERY RECANALIZATION AFTER THROMBOLYSIS IN ACUTE STROKE}

M. Millán, J.F. Arenillas, M. Rodríguez-Yáñez, N. Pérez de la Ossa,

P. Sandoval, Y. Silva, L. Dorado, C. Guerrero, A. Dávalos

Hospital Universitari Germans Trias i Pujol, Badalona (Barcelona), Spain

Background: Hyperglycemia (HG) has been associated with an antifibrinolytic effect and resistance to early (within 2 hours) arterial recanalization after thrombolysis. We aimed to study the effect of $\mathrm{HG}$ on early and late recanalization of middle cerebral artery (MCA) occlusion in patients treated with tissue plasminogen activator (tPA)

Methods: We prospectively studied 85 ischemic stroke patients (median age 68, NIHSS 14) treated with IV tPA within 3 hours from symptoms onset who showed MCA occlusion on prebolus transcranial Duplex (TCDx) examination. TCDx follow-up was performed at 2, 6, 12 and 24 hours after tPA. Thrombolysis in Brain Ischemia (TIBI) grades were used to define complete, partial or absent MCA recanalization. Baseline serum glucose and plasma glycosylated hemoglobin $(\mathrm{HbA} 1 \mathrm{c} \%$ ) levels were recorded. HG was defined as serum glucose $>150 \mathrm{mg} / \mathrm{dL}$ Results: Complete or partial MCA recanalization was achieved in $50.6 \%$ of patients at 2 hours, $60.8 \%$ at 6 hours, $72.7 \%$ at 12 hours and $84.7 \%$ at 24 hours. $\mathrm{HG}$ at baseline was found in $20(23.5 \%)$ patients. HG was associated with lower recanalization rates at $2(11.9 \%$ vs $36.6 \%, \mathrm{p}=0.009)$ and $6(14.6 \%$ vs $35.5 \%$, $\mathrm{p}=0.031$ ) hours, but no differences were found at 12 and 24 hours. No other baseline variables, including $\mathrm{HbA1c}$, were associated with early or late resistance to MCA recanalization. Acute $\mathrm{HG}$ remained as an independent predictor of no recanalization at 2 hours $(\mathrm{OR}, 4.89 ; 95 \% \mathrm{CI} 1.4-15.9, \mathrm{p}=0.009)$ and 6 hours $(\mathrm{OR}$, $3.45 ; 95 \%$ CI 1.1-10.7, $\mathrm{p}=0.032$ ) after adjustment for age, stroke severity and proximal MCA occlusion. No significant effect was observed beyond 6 hours

Conclusion: These findings confirm that HG is associated with resistance to MCA recanalization up to 6 hours after thrombolysis in ischemic stroke patients

\section{Acute stroke: treatment concepts III}

INTRAARTERIAL TREATMENT FOR ACUTE ANTERIOR CIRCULATION ISCHEMIC STROKES DUE TO INTRACRANIAL LARGE VESSEL OCCLUSION BEYOND 8 HOURS - PRELIMINARY RESULT

S. Zaidi, R. Lin, N. Vora, R. Gupta, V. Reddy, M. Hammer, K. Uchino,

L. Wechsler, A. Aleu, T. Jovin

University of Pittsburgh Stroke Institute, Pittsburgh, PA, USA

Background: Selection of patients with acute ischemic stroke (AIS) for endovascular therapy is currently based on strict time window. We sought to determine the treatment effects of endovascular therapy on AIS patients presenting beyond 8 hours of symptom onset, when selection is based on pathophysiological characteristics assessed through neuroimaging.

Methods: We retrospectively identified AIS patients with carotid terminus, middle cerebral artery M1 and/or M2 division occlusion treated with endovascular modalities from $01 / 2003$ to $12 / 2007$ at our center. We classified patients into $0-8 \mathrm{hr}$ vs $>8 \mathrm{hr}$ groups, based on time from symptom onset to first diagnostic angiographic run. All patients treated beyond 8 hrs had large mismatch on imaging (MR or CT perfusion) prior to intervention. Patient demographics (age, sex), risk factors, thrombus site, baseline National Institute of Health Stroke Scale (NIHSS) score, and treatment modality (IA tPA, angioplasty, stent, and/or MERC embolectomy device) were compared between the groups. Endpoints are outlined in the table.

Results: Of 160 consecutive patients, 37 (23.1\%) were treated after 8 hrs. Patients in $0-8 \mathrm{hr}$ treatment group had a higher mean baseline NIHSS score (18 vs 12 , $\mathrm{p}<0.05$ ); more patients in the $>8 \mathrm{hr}$ group were treated with MERCI device (57 vs $41 \%$, p0.05) and stenting (51.4 vs $29.3 \%$, p0.003). No significant differences were found with respect to endpoints between the two groups, although a trend towards lower incidence of parenchymal hematoma $(\mathrm{PH})$ and lower final diffusion weighted image (DWI) infarct volumes were detected in the $>8 \mathrm{hr}$ group (see table).
Study endpoints

\begin{tabular}{lccc}
\hline Endpoints & $0-8$ hr Group & $>8$ hr Group & \\
\hline Discharge - home or rehab & $66 \%$ & $60 \%$ & $\mathrm{p}=0.82^{*}$ \\
PH & $15.3 \%$ & $8.3 \%$ & $\mathrm{p}=0.4^{*}$ \\
DWI infarct volume & $101.2(96.3)$ & $83.1(64.6)$ & $\mathrm{p}=0.27^{*}$ \\
Inpatient mortality & $30.8 \%$ & $20 \%$ & $\mathrm{p}=0.29^{*}$
\end{tabular}

*Fisher exact test; **t-test.

Discussion: Imaging may identify AIS patients beyond 8 hrs from symptom onset who can achieve good outcomes with endovascular treatment. Furture prospective trials are needed.

\section{Acute stroke: treatment concepts III}

INTRA-ARTERIAL ADMINISTRATION OF MICROBUBBLES AND CONTINUOUS 2-MHZ ULTRASOUND INSONATION TO ENHANCE INTRAARTERIAL THROMBOLYSIS

M. Ribo, C.A. Molina, B. Alvarez, M. Rubiera, J. Alvarez-Sabin, M. Matas Unitat Neurovascular, Hosptial Vall d'Hebron, Barcelona, Spain

Microbubbles (MB) and ultrasound have shown to enhance thrombolytic effect of iv-tPA. We sought to evaluate the safety and efficacy of local MB administration during intra-arterial thrombolysis (IA) and continuous transcranial Doppler (TCD) monitoring on middle cerebral artery (MCA) recanalization.

Methods: Patients with acute proximal MCA occlusion were treated with IA tPA (repeated $4 \mathrm{mg}$ up to $20 \mathrm{mg}$ or recanalization) and IA galactose based MB (up to 3 doses of $1 \mathrm{ml}(400 \mathrm{mg} / \mathrm{dl}))$. MBs and tPA were infused directly intra-clot through a microcatheter. TCD monitoring allowed continuous insonation at clot location. Retrieving devices were not used. Recanalization was angiographically assessed according to TICI reperfusion score and compared to simultaneous online TCD data. IA procedures were always stopped 6 hours after symptoms onset. Recanalization was reassessed with TCD at 12 hours after symptoms onset. Hemorrhagic transformation (HAT) was assessed at 24 hours. Neurological status was assessed with the NIHSS. At three months patients were considered independent if $\mathrm{mRS}$ was $\leq 2$.

Results: Of the 9 included patients, 7 received standard iv.tPA prior to IA rescue. Median pre-IA NIHSS was 20. Median time to IA initiation was: 175 minutes. Mean IA administered doses were: tPA $10 \mathrm{mg}$, MB $1 \mathrm{ml}$. TCD allowed direct visualization of massive $\mathrm{MB}$ arrival during every dose administration. After MB infusion, recanalization was confirmed in 7 patients $(78 \%)$ : complete-TICI3: 2 patients $(22 \%)$, partial-TICI2: 5 patients $(56 \%)$. A perfect correlation was observed between TICI and TCD scores. At 12 hours recanalization rate was still $78 \%$, however complete recanalization increased to $56 \%$. One patient $(11 \%)$ experienced symptomatic HAT accounting for the only death. Median NIHSS evolution was: 12 at 24 hours and 10 at discharge. At 3 months 4 patients (44\%) were independent.

Conclusion: Ultrasound-activated intraclot-delivered MB and tPA may be a safe strategy to enhance the thrombolytic effect and increase recanalization rates. Ongoing study: Presented data will include all future treated patients.

\section{Epidemiology of stroke II}

\section{Epidemiology of stroke II}

RT-PA AND HEPARIN IN THE LYON RT-PA PROTOCOL: BENEFICIAL EFFECT OF INTRAVENOUS HEPARIN ON OUTCOME AND INTRACEREBRAL BLEEDING AT SPECIFIC TIMES OF INITIATION (200 PATIENTS)

P. Trouillas, L. Derex, F. Philippeau, S. Cakmak, N. Nighoghossian, L. Zeng, M. Hermier, J.C. Froment, M. Hanss

Cerebrovascular Unit, Hôpital Neurologique, Lyon, France

The issue of the adjunctive role of intravenous unfractionated heparin (IVUFH) in cerebral rt-PA intravenous thrombolysis remains pending, although heparin is generally banned for the first 24 hours in the guidelines. The LYON rt-PA protocol may give indicative data on this issue

Patients and methods: Two hundred consecutive patients were submitted to 4 successive heparin regimens, including consecutive patients: immediate IVUFH, IVUFH at $12 \mathrm{~h}$, IVUHF at $24 \mathrm{~h}$ after a non-hemorrhagic CT Scan and low molecular weight nadroparin at any time. The aPTT objective for the 3 IVUFH subgroups was 45 seconds, with a duration objective of 1 week. 
Results: The 4 groups had non significant differences for baseline severity score, age and interval. Immediate heparin $(n=31)$ resulted in a parenchymal hematoma $(\mathrm{PH})$ rate of $9.6 \%$, while heparin at $12 \mathrm{~h}(\mathrm{n}=10)$ and at $24 \mathrm{~h}(\mathrm{n}=66)$ resulted each in a $\mathrm{PH}$ rate of $0 \%$. Nadroparin $(\mathrm{n}=78)$ resulted in a $\mathrm{PH}$ rate of $14 \%$ and in a high early $\mathrm{PH}$ rate $(8.9 \%)$. Concerning prognosis, immediate heparin resulted in a mRS 0,1 rate of $41.9 \%$, while heparin at $24 \mathrm{~h}$ resulted in a mRS 0,1 rate of $51.5 \%$ and heparin at 12 hours of $70 \%$. Conversely, nadroparin was associated with a poor prognosis (mRS 0,1 rate: $16.7 \%$ ). Use of IVUHF vs nadroparin was an independent factor of good prognosis (OR 2.61 95\% CI 51.08-6.30).

Discussion: These results indicate that immediate IVUHF enhances early postthrombolytic pro-hemorrhagic processes without outcome benefit. Conversely, IVUHF at $24 \mathrm{H}$, after non-hemorrhagic CT Scan, results in a good prognosis effect and is extremely safe for $\mathrm{ICH}$, even showing an unexpected anti-hemorrhagic effect.

Conclusion: Intravenous heparin at 24 hours with an aPTT objective of $45 \mathrm{~s}$, after a non hemorrhagic CT Scan, is safe and probably efficient in amplifying the rt-PA thrombolytic effect and preventing late reocclusion due to rt-PA induced thrombin generation.

\section{Epidemiology of stroke II}

IMAGING OF HYPOXIC-ISCHEMIC PENUMBRA WITH

18F-FLUOROMISONIDAZOLE AND RELATED GLUMATATE LEVELS IN

ANEURYSMAL SUBARACHNOID HEMORRHAGE: PRELIMINARY RESULTS

A. Sarrafzadeh, M. Plotkin, M. Czabanka, P. Vajkoczy

Charité Campus Virchow Medical Center, Berlin, Germany

Background and purpose: Infarction following vasospasm or a concomitant in tracerebral hemorrhage is a severe complication mainly contributing to the high morbidity and mortality in patients with aneurysmal subarachnoid hemorrhage (SAH). The most extensively investigated and validated positron emission tomography radiotracer of hypoxia to date is (18)F-fluoromisonodazole ((18)F-FMISO) It allows early imaging of the target of therapy being the severely hypoxic but salvageable tissue. We investigated the excitotoxic mediator and marker of ischemia glutamate, monitored with microdialysis (MD), in relation to 18F-FMISO PET findings in patients with aneurysmal SAH.

Methods: Seven SAH patients (WFNS grade I-III, age 48.9 yrs) were prospectively studied with microdialysis monitoring and 18F-FMISO PET. The MD-catheter was inserted into the brain parenchyma of interest. Extracellular levels of glutamate were analyzed hourly for eight days. 18F-FMISO PET was performed within the period of occurrence of possible vasospasm (day 3-8 after SAH).

Results: In asymptomatic patients $(n=3)$ no hypoxic region was found and glutamate levels were low $(<10 \mathrm{mMM})$. Symptomatic patients presenting acute $(\mathrm{n}=3)$ or delayed $(n=1)$ neurological deficits had higher glutamate concentrations $(10-35$ $\mathrm{mM}$ ). 18F-FMISO-uptake was increased in two of these cases within the microdialysis region of interest. When infarction was visible on computerized tomography $(\mathrm{n}=2)$, no uptake was observed.

Conclusion: 18F-FMISO-PET is a promising method to early diagnose metabolically compromised lesioned brain tissue and possibly allows to image the part of salvageable tissue for future targeted therapy.

\section{Epidemiology of stroke II}

\section{LONG-TERM MORTALITY, MORBIDITY AND HOSPITAL CARE}

FOLLOWING STROKE: A 10-YEAR COHORT STUDY

A.R. Finlayson, J.L. Bishop

Information Services, National Health Service Scotland, Edinburgh, United Kingdom

Background and purpose: Stroke is one of the leading causes of death and reason for admission to hospital in Scotland. This study assessed survival, morbidity and cost of hospital care over ten years following a first-ever stroke.

Methods: Patients with a first ever admission to hospital for stroke in 1996 were identified from routinely collected hospital data in Scotland. Patient characteristics (age, sex, length of stay, hospital) were analysed and long-term survival, morbidity, and treatment provided in hospitals were evaluated. Follow up outcome analyses were made possible by the record linkage between hospital records and to death registration records, which is carried out routinely in Scotland.

Results: A total of 8368 patients diagnosed with a first stroke in 1996 were identified. Mean age was 73.4 years (SD 12.3) at stroke onset. In-hospital mortality was $29.7 \%, 32.4 \%$ at 90 days, $41.8 \%$ at 1 year, while $80.1 \%$ were dead within ten years. Mean number of days spent in hospital on initial admission was 19 days, rising to 77 days in total over the 10 years. $14 \%$ of patients were readmitted for stroke at least once over the next 10 years with a mean number of $1.8 .56 \%$ of patients were readmitted to hospital for any reason and the mean number of readmissions over the 10 years was 6.4 .

Conclusion: Patients suffering first onset of stroke require significant hospital resources, both during their initial hospital admission and throughout subsequent admissions. Although many people do not survive their initial onset of a stroke those that do go on to have many more stroke related admissions. Most of these patients are elderly and as the number of elderly people in the population and the survival rate from initial stroke increases we can reasonably expect the amount of resources required by hospitals to cope with stroke admissions to rise.

\section{Epidemiology of stroke II}

\section{YEAR TRENDS IN CEREBROVASCULAR DISEASE MORTALITY - THE} EFFECT OF RURALITY AND DEPRIVATION IN SCOTLAND

J.L. Bishop, A. Redpath

Information Services, National Health Service Scotland, Edinburgh, United Kingdom

Objectives: Cerebrovascular Disease (CVD) is the third main cause of death in Scotland and a leading cause of premature mortality. Scotland does not compare favourably with Western Europe and CVD became a priority for the Scottish Government. This study looks at 10 year trends in CVD premature mortality and determines whether rates vary by rurality and deprivation.

Methods: Death registration data was used to calculate age-adjusted mortality rates from cerebrovascular disease for ages under 74 from 1995 to 2006. Area based indices of deprivation (quintiles) and rurality (urban or rural) were assigned based on address. Of particular interest was the deprived rural population which has long been assumed to be the most disadvantaged in Scotland. 3 year rolling averages of the rates were examined. Rates were adjusted for age and sex.

Results: CVD premature mortality across all deprivation and urban/rural categories decreased in Scotland by 38\% between 1997 and 2006 years and is on track to meet the 15 year $50 \%$ target reduction by 2010 . However, for the first time there was a small increase in the male mortality rate from 23.0 per 100,000 population in 2005 to 23.6 per 100,000 population in 2006 . Rates amongst deprived populations remained consistently higher than rates amongst affluent populations. The inequality ratio between the most and least deprived populations actually increased over the 10 year period from 2.5 to 2.8 . Rates in urban populations were slightly higher than those in rural populations but we did not find any evidence of significant differences in rates between urban and rural populations within deprivation quintiles.

Conclusions: Deprivation is the leading factor in differences in CVD premature death rates and despite the work being done by the Scottish Government, vas inequalities still remain. Whether a person lives in an urban area or a rural area does not seem to have any major effect. Although small in 2006,the upturn in male premature mortality requires further vigilance.

\section{Epidemiology of stroke II}

\section{INCIDENCE, RISK FACTORS, SEVERITY AND EARLY OUTCOME OF} TOAST-DEFINED ISCHAEMIC STROKE SUBTYPES - THE NORTH DUBLIN POPULATION STROKE STUDY

M. Marnane, O. Sheehan, A. Merwick, L. Kyne, A. Moore,

P.M.E. McCormack, D. Harris, N. Fitzsimon, L. Daly, P.J. Kelly

Neurovascular Clinical Science Unit, Mater University Hospital and University College Dublin, Dublin, Ireland

Background: Large prospective population studies are important to accurately determine the frequency and natural history of ischaemic stroke while avoiding potential selection bias that may occur in hospital-based studies. Although the mechanism-based TOAST classification of stroke subtype is frequently used in observational studies and clinical trials, relatively little population data exists.

Methods: The North Dublin Population Stroke Study is a prospective cohort study of stroke and TIA in 294,592 individuals over one year. Multiple validated overlapping ('hot' and 'cold' pursuit) sources of case ascertainment were used. Ischaemic stroke was classified according to TOAST subtype by a trained Stroke Physician using a standard data abstraction and classification algorithm. NIH Stroke Scale (NIHSS) and modified Rankin score (RS) were performed within 72 hours, and RS repeated at 7 and 28 days.

Results: 455 new (first-ever and recurrent) ischaemic stroke cases were ascertained. Crude subtype-specific incidence rates (per 100,000) were: Large artery (LA) 15, Cardioembolic (CE) 55, Small artery (SA) 20, Other 3, Undetermined 62. Patients with CE were older $(\mathrm{p}=0.0005)$ and more likely female $(\mathrm{p}=0.008)$ than those with LA or SA stroke. LA stroke was more common in males $(\mathrm{p}=0.008)$. Hypertension and diabetes mellitus were equally common. CE and LA stroke had greater NIHSS and RS than SA stroke at all time-points $(\mathrm{p}<0.01$ for all). No differences in stroke 
severity, recurrence, or mortality at 7 or 28 days were present between CE and LA subtypes.

Conclusions: TOAST-defined subtypes were associated with distinct frequency, risk factor and outcome profiles. Improved prevention of CE and LA stroke is likely to provide most benefit in reduction of stroke-related disability and mortality.

\section{Epidemiology of stroke II}

\section{DISABILITY-ADJUSTED LIFE YEARS LOST FROM STROKE AND PER} CAPITA GROSS DOMESTIC PRODUCT IN SOUTH-EAST ASIA: AN

\section{ECOLOGICAL STUDY}

N. Venketasubramanian, R.N. Gan

National Neuroscience Institute, Singapore, Singapore

Introduction: Stroke is a major cause of death and disability in many countries of the world. South East Asia (SEA), home to 535 million people, comprises 10 developing countries with widely differing wealth status.

Objective: This paper studies the association between disease burden due to stroke and per capita wealth along countries in SEA.

Methods: Disease burden was measured in disability-adjusted life years lost (DALYs)/100 000 population due to stroke, age and sex-standardised to the World Standard Population. Wealth was measured in per capita gross domestic product (GDP) in International dollars (Int \$). Data on DALYs and per capita GDP for the 10 SEA countries was obtained from the World Health Organisation (WHO) website (http://www.who.int/en/). The data of each country was plotted as continuous variables. Pearson's correlation $r$ was determined. Data was analysed using SPSS for Windows ver 12.0

Results: Latest data available was for 2002. DALYs due to stroke ranged from 1 in Brunei to 1311 in Indonesia, reflecting in part the size of the population in these countries, as Brunei has the smallest and Indonesia the largest population in SEA. Age-sex standardised DALYs ranged from 251/100000 in Singapore to $991 / 100000$ in Laos. Values were in the lower range for Thailand, Malaysia and Brunei, and in the higher range for Khmer, Myanmar and Indonesia, with intermediate values for Philippines and Vietnam. There was an inverse exponential relationship between DALYs and GDP. When both data were log transformed, a linear relationship was obtained, $\mathrm{r} 2=0.89$.

Conclusion: There is a strong inverse relationship between stroke burden and wealth in SEA countries. This study suggests that countries should strive to raise their wealth so as to reduce their disease burden due to stroke.

\section{Epidemiology of stroke II}

\section{NO EVIDENCE FOR A ROLE OF THYROID AUTOIMMUNITY IN THE} PATHOGENESIS OF CERVICAL ARTERY DISSECTIONS

C. Lichy, C. Becker, M. Kloss, M. Arnold, T.B. Brandt, A. Pezzini,

C.G. Grond-Ginsbach

University of Heidelberg, Heidelberg, Germany

Background: A recent study suggested a role of antithyroid autoimmunity in the process of local inflammation related to spontaneous cervical artery dissection (sCAD). Presence of thyroid antibodies was found in 9 of 29 sCAD patients, but only in 2 of 29 patients with stroke of other etiologies. We aimed to confirm this finding by investigating an independent larger group of patients.

Methods: In 72 consecutive patients with MRI-confirmed sCAD (29 women, mean age 43.1 years), serum tests for antibodies against thyreoglobulin ( $\mathrm{TgAb}$ ), thyroid peroxidase (TPOAb), and thyroid-stimulating hormone receptor (TRAb) were performed. Additionally, TSH levels were measured in all patients and a systematic evaluation for clinical signs of thyroid disease was done. We used data of a historical control group of 6.850 healthy Caucasian subjects to estimate the significance of potential findings.

Results: In $12(16.7 \%$ ) patients ( 8 women, mean age 44.8 years) had TgAb and/or TPOAb levels above the upper limit of normal (4 TgAb, 2 TPOAb, 6 both). However, this is much lower than reported by the initial study and is within the range of thyroid-antibody positivity found in the healthy population $(12.9 \pm 0.6 \% \mathrm{TgAb}$, $14.3 \pm 0.4 \% \mathrm{TPO} \mathrm{Ab})$.

No patient showed clinical evidence for thyroid disease. TSH levels were normal in all patients. One patient had a history of Grave's disease, but none had increased levels of TRAb.

Discussion: In our study on the prevalence of antithyroid-antibodies in SCAD, we could not confirm a previously suggested role of antithyroid autoimmunity in the pathogenesis of this vasculopathy.
8 Epidemiology of stroke II

GENDER DIFFERENCES IN PATIENTS WITH ACUTE ISCHEMIC STROKE

V. Caso, F. Corea, W. Ageno, A. Lanari, L. Bertolani, G. Comi, P. Previdi, G. Silverstrelli, A.M.R. Billeci, M. Paciaroni

University of Perugia, Ospedale S. Maria della Misericordia, Perugia, Italy

Background: Population-based studies have shown that women, at any age, have a lower risk of stroke than men; but the risk of stroke increases with advancing age and women on average today live 5 to 10 years longer than men, so their overall burden of stroke is greater. However, a reduced diagnostic work-up in women affected by stroke has been evidenced as well as an undertreatment of complications compared to men.

Methods: Data of consecutive patients included in a multicenter-study were analyzed from 1st of January 2006-30thof April 2007). Vascular risk factors, clinical presentation, NIHSS at admission, pre-treatment and stroke treatment during hospitalization and disability/death $(\mathrm{mRS}>3)$ at three months were compared in men and women.

Results: A total of 1125 strokes were observed (women:494, 44.9\%). The mean age was $76.36 \pm 12.93$ for women and $70.07 \pm 12.95$ for men $(\mathrm{p}<0.001)$. The vascular risk factors such as smoking $(26.4 \mathrm{v} 11.1 \%, \mathrm{p}<0.001)$, alcohol abuse $(5.3 \mathrm{v} 0 \%$, $\mathrm{p}<0.001)$ and PAD $(3.6 \mathrm{v} 1.1 \% . \mathrm{p}=0.02)$ were higher in men, while women tended to have more atrial fibrillation $(22.2 \% \mathrm{v} 17.1 \%, \mathrm{p}=0.01)$. The mean baseline NIHSS was 9.47 for women and 7.67 for men $(\mathrm{p}=0.001)$. Women tended to have more TACI $(19.4 \mathrm{v} 11.2 \%, \mathrm{p}<0.001)$ and more cardioembolic stroke $(30.4 \mathrm{v} 23.2 \%, \mathrm{p}=0.04)$ No differences in previous anticoagulation treatment and acute thrombolitic therapy had been observed. At three months more women were dead or disabled $(46.6 \% \mathrm{v}$ $36.1 \%, \mathrm{p}=0.001$ ). However, female gender was not an independent factor for bad outcome in regression analysis, while older age, higher NIHSS-score and diabetes were predictors for bad outcome.

Conclusions: Female gender is not an independent factor for bad outcome. However, women tend to be older than men and to have more severe stroke.

\section{Epidemiology of stroke II}

\section{CASE FATALITY AND DISABILITY OF 3123 CONSECUTIVE PATIENTS IN CHENGDU STROKE REGISTER}

M. Liu, W. Li, Y. Fang, B. Wu

West China Hospital, Sichuan University, Chengdu, China

Background and purpose: Community based stroke studies in China were mainly conducted in 1980s and have methodological limitations. Few prospective, consecutive and long term follow up hospital based stroke register was conducted in mainland China. No disability data were reported from community based or hospital based registers in China. This study analyzed data from Chengdu Stroke Register to provide information on short and long term case fatality and disability in stroke.

Methods: We conducted a pilot study in 1998 to explore the feasibility. Since Mar 1, 2002 we have been registering prospectively stroke patients consecutively admitted to our hospital. The World Health Organization definition for stroke was used. Patients with stroke occurred within one month were included. The baseline demographic, risk factors, treatment, and outcome data were recorded. We followed up patients at 7 days, 1, 3, 6 and 12 months for death, disability and recurrence. Disability is defined as Modified Rankin Scale scored $\geq 3$.

Results: A total of 3123 patients registered from Mar 1, 2002 to Aug 31, 2006 were included. Age ranged from 14 to 98 years and the mean age was $63 \pm 18$ years. Male patients accounted for $60 \% .97 \%$ of the patients had brain CT or MRI scanning. The length of hospital stay ranged from 1 day to 113 days and the average is $11 \pm 9$ days. Ischemic stroke accounted for $62 \%$, intracranial hemorrhage $28 \%$, subarachnoid hemorrhage $4 \%$, TIA $6 \% .10 \%$ of the ischemic stroke patients arrived in hospital within 3 hours of stroke onset and 22\% within 6 hours. Only $0.9 \%$ of the patients with ischemic stroke were treated with intravenous thrombolysis. $3 \%$ of the ischemic and $11 \%$ of the hemorraghic stroke patients died within 7 days, $5 \%$ and $14 \%$ at one month. $12 \%$ and $19 \%$ at 12 months. Death or disability were $37 \%$ and $40 \%$ at 3 months, $33 \%$ and $39 \%$ at 12 month in ischemic and hemorraghic stroke patients.

Conclusions: Short and long term case fatality and disability rates are relatively low in this group of patients. The reason is unclear and need further study. 


\section{Recovery and rehabilitation}

\section{Recovery and rehabilitation}

SAFETY IN THE FIRST 170 PATIENTS OF A VERY EARLY REHABILITATION TRIAL (AVERT PHASE III)

J. Bernhardt, H. Dewey, J.M. Collier, A. Thrift, R. Lindley, M. Moodie,

G. Donnan, on behalf of the AVERT Collaboration

National Stroke Research Institute, Melbourne, Victoria, Australia

Objectives: Very early mobilisation (VEM) may be an important component of effective stroke unit care. The Phase III AVERT trial will test the efficacy and cost-effectiveness of this simple intervention. We report the safety results from the first 170 patients completing 3 month assessments.

Methods: Study design: Phase III randomized controlled trial of 2104 patients, with blinded outcome assessments. Inclusion criteria: Patients over 18 years, admitted within $24 \mathrm{hrs}$ of confirmed cerebral infarct or haemorrhage, whose physiological parameters fall within set limits. Exclusion Criteria: Patients with severe premorbid disability, severe comorbidities or requiring palliative care. Treatment with IPA is not an exclusion. Randomisation: Web-based, blocked randomisation by site and stroke severity (NIHSS). Intervention: The VEM protocol, delivered by a nurse/physiotherapy team, begins within $24 \mathrm{hrs}$ of stroke and continues for up to 14 days. Control patients receive standard care (SC). Safety outcomes: Deaths and serious adverse events (SAEs), including serious falls, at 3 months.

Results: Phase III commenced in July 2006 in nine acute stroke units across Australia. To January 2008, 170 patients have completed 3 month assessments. Mean age 70.2 (SD 14.0) years, $80.6 \%$ with first ever stroke, $83.5 \%$ infarct, mean NIHSS score of 8.4 (SD 6.1), 40\% with NIHSS $>7$ (moderate and severe stroke). To date 19 patients $(11.2 \%)$ have been treated with tPA, $63 \%$ of whom had experienced a TACI. Only $10(5.8 \%)$ patients have died within 3 months of stroke, four having received tPA. Thirty eight SAEs have been reported including 8 stroke progressions and 7 recurrent strokes. There has been only one serious fall with fracture reported.

Conclusion: VEM within a 24 hour time window appears safe with an acceptable rate of serious adverse events and only 5.8\% deaths occurring in the first 170 patients.

\section{Recovery and rehabilitation}

MOTOR NETWORK CHANGES ASSOCIATED WITH SUCCESSFUL MOTOR SKILL RELEARNING AFTER ACUTE ISCHEMIC STROKE. A LONGITUDINAL FUNCTIONAL MRI STUDY

T. Askim, B. Indredavik, T. Vangberg, A. Håberg

St. Olavs Hospital, Trondheim University Hospital and Norwegian University of Science and Technology, Trondheim, Norway

Background: Brain plasticity after stroke has been studied extensively, but results are contradictory. Motor learning mechanisms may be operative in stroke recovery and possibly reinforced by rehabilitative training. In the present study we wanted to assess the association between motor skill relearning and early motor network changes after acute ischemic stroke.

Methods: Twelve patients with first-ever stroke not encompassing the primary sensorimotor cortex, treated in an evidence based comprehensive stroke unit with emphasize on intensive rehabilitation, were assessed with functional MRI and clinical tests, such as the upper limb items of Motor Assessment Scale (UL-MAS), Nine Hole Peg Test (NHPT), transversal grip strength (TVGS) and key grip strength (KGS), within the first week after stroke and three months later. Statistical parametric mapping was used for statistical inferences of fMRI data.

Results: All patients, except one, fulfilled the criteria for complete recovery of the affected arm and hand. Improvement in UL-MAS correlated positively with increased activation within the area of contralesional SII and SMA, plus ipsilesional cerebellum. Increased activation in contralesional MII, SI, SII, bilateral SMA and cerebellum correlated with increased speed on NHPT. The improvement in TVGS was mainly associated with increased activation in ipsilesional somatosensory association areas, contralesional SII and insula, bilateral thalami and cerebellum, while for KGS the activated regions were bilateral MI and SMA.

Discussion: The recruitment of contralesional cortical structures is considered to represent cortical plasticity involved in successful relearning of dexterity while the bilateral activation seems important for recovery of grip strength after ischemic stroke.
3 Recovery and rehabilitation

5000 FINGER GRIP MOVEMENTS WITH A NEW ROBOTIC HAND REHABILITATION DEVICE - EFFECTS ON GRIP ABILITIES AND FUNCTIONAL MRI

C. Enzinger, P. Grieshofer, G. Reiter, R. Scherer, R. Linderl-Madrutter, C. Pargfrieder, S. Pegritz, W. Wurm, C. Neuper, F. Fazekas

Medical University Graz, Graz, Austria

Background: Successful rehabilitation of highly impaired finger and hand movements after stroke is challenging and often remains unsatisfactory with conventional therapies. We therefore developed a new mechatronic device for rehabilitation of hand function which allows delivering well characterised, high frequent, repetitive movement sequences in an individualised manner. The goal of the ongoing exploratory study is to assess the consequences of such training both directly regarding gains in performance and indirectly using functional MRI.

Methods: To date, 11 stroke patients with a moderate to high-grade paresis of the upper limb (pinch grip force grades 2 or 3 according to MRC; spasticity according to the Ashworth Scale $<3$ ) have been included (mean age 62, range 47-78, interval to their stroke 41 to 434 days). Before and after three weeks of standardised training using a hand robot (developed at the clinic Judendorf-Strassengel) with an average of 5000 grip movements during 15 therapy sessions in addition to conventional physiotherapy, patients were both tested behaviourally and a subset underwent repeated fMRI using an identical paradigm at 3T. The fMRI experiment consisted of active and passive flexion and extension of the digits II-V of both hands. The Motricity Index (M.I.) before and after training and force measurements on the robot during each session served to assess gains in functional strength.

Results: Following the robot training, we observed significant improvements of the patients' functional strength, both clinically and by measurements of force using the device (M.I. pinch grip pre $23.3 \pm 6.6$ vs. post $26.3 \pm 4.6, p=0.03$; finger flexion pre $7.75 \pm 4.5 \mathrm{~N}$ vs. post $11.9 \pm 4.8 \mathrm{~N}, \mathrm{p}=0.04$ ). At baseline, movement of the paretic hand vs. rest was associated with activation of a more bihemispheric network with additional recruitment of ipsilateral motor areas in the undamaged hemisphere compared to movement of the unaffected hand. Subsequent to robot therapy, significant increases in activation in the cerebellum and basal ganglia were noted with movement of the paretic, trained hand. fMRI activation patterns with movement of the healthy (not-trained) hand vs. rest did not change (robust activation of contralateral primary sensorimotor cortex, supplementary and cingulate motor areas, ipsilateral cerebellum in expected somatotopy)

Conclusion: These preliminary results suggest distinct changes in sensorimotor networks associated with robotic-assisted rehabilitation of hand function after stroke. The causes and clinical significance of these changes will need further exploration.

\section{Recovery and rehabilitation}

THE EFFECTS OF FUNCTIONAL STRENGTH TRAINING ON LOWER LIMB STRENGTH AND FUNCTION AFTER STROKE

E.V. Cooke, R.C. Tallis, S. Miller, V.M. Pomeroy

St. George's University of London, London, United Kingdom

Background: Stroke survivors often have permanent residual motor impairment This may be due to a sub-optimal dose of conventional physical therapy (CPT) and the discouragement of strength training. However, experimental evidence suggests that strength training might be beneficial. Hypothesis Adding functional strength training (FST) to CPT improves muscle function and gait more than either CPT alone or increased intensity of CPT

Methods: Multi-centred randomised controlled observer-blind trial. Subjects were within 3 months of stroke with ability to voluntarily move their paretic lower limb. A power calculation estimated sample size as 102. Subjects underwent baseline measurements before being allocated randomly to: $\mathrm{CPT}$; or $\mathrm{CPT}+\mathrm{CPT}$; or CPT+FST for 6 weeks. All additional therapy was provided up to 1 hour/day, 4 times/week. Outcome measures were made at 6 weeks after baseline. Measurement battery included: muscle strength (maximum torque during isokinetic knee flexion); walking speed; and functional mobility (Rivermead). Analysis followed the intention-to-treat principle. Data was tested for differences between groups using the Kruskal-Wallis test.

Results: 109 subjects were recruited. Mean age was 68.3 (SD12.03) years. The attrition rate was $8.3 \%$. For muscle strength the median (IQR) change following intervention was $4.17(9.7) \mathrm{Nm}$ for CPT, 6.33 (12.7) $\mathrm{Nm}$ for CPT+CPT and 10.57 (17.5) $\mathrm{Nm}$ for $\mathrm{CPT}+\mathrm{FST}(\mathrm{p}=0.11)$. Median (IQR) change in walking speed following intervention was $0.03(0.13) \mathrm{m} / \mathrm{sec}$ for CPT, $0.19(0.44) \mathrm{m} / \mathrm{sec}$ for CPT+CPT and $0.09(0.17) \mathrm{m} / \mathrm{sec}$ for CPT+FST $(\mathrm{p}=0.10)$. Rivermead median (IQR) score following intervention was 39.5 (20.5) for CPT, 41.0 (12.0) for CPT+CPT and 41.0 (8.50) for CPT+FST $(\mathrm{p}=0.255)$ 
Discussion: Immediately after intervention no statistically significant differences were found between groups for muscle strength, walking speed or functional mobility. There was, however, a tendency for higher change scores in $\mathrm{CPT}+\mathrm{CPT}$ and CPT+FST groups.

\section{Recovery and rehabilitation}

\section{STUDIED ON LANGUAGE NETWORKS DAMAGED OF WERNICKE'S APHASIA BY IMAGING \\ Z. Yumei, Z. Xingquan, W. Chunxue, W. Yongjun \\ Beijing Tiantan Hospital, Capital Medical University, Beijing, China}

Background and purposes: There are a variety of differing types of aphasia classified by the patient's deficit in language ability. Among them, Wernicke's aphasia is known as a fluent aphasia, the major impairment is semantic. As the Geschwind-Lichtheim model, Wernicke's aphasia damaged Brodmann 22 and 39 areas, namely Wernicke's area. We used MR diffusion tensor imaging (DTI) and diffusion tensor fiber tractography (DT) to study Wernicke's area on volunteers and Wernicke's aphasia cases in order to offer functional anatomy results for language disorders.

Methods: DTI and DTFT were implemented on Simens 3.0T MR system. Data were acquired in twenty volunteers and twenty Wernicke's aphasia cases. Brodmann 22, Brodmann 39 areas and right hemisphere corresponding areas were performed as regions of interest (ROIs), All fibers passing through these ROIs were reconstructed in three dimensions and visualized streamtubes. And we observed Wernicke's area fibers distributing both volunteers and Wernicke's aphasia cases, measured fractional anisotropy (FA) value.

Results: From the results of volunteers, we found that Brodmann 22 area and 39 area fibers/FA in left hemisphere had no significant different compared with that of the mirror side $(\mathrm{p}>0.05)$. On the other hand, we also found that Brodmann 22 and 39 areas in left hemisphere of Wernicke's aphasia had lesser fibers than that of mirror side $(\mathrm{p}<0.05)$.

Conclusions: The language networks of Wernicke's aphasia were damaged, and DTI, DTFT can be used to elucidate and investigate major nerve pathways in the brain, and provide future information on the function of the normal and abnormal human brain.

\section{Recovery and rehabilitation}

\section{UPPER PLEXUS ANAESTHESIA IN REHABILITATION OF HAND FUNCTION} IN SUBACUTE STROKE

K.M. Stephan, M. Stollorz, J. Bohland, J. Specht, H. Krause, T. Steinforth, V. Hömberg

St. Mauritius Therapieklinik Meerbusch, Augusta Krankenhaus Düsseldorf, NTC Universität Düsseldorf, Meerbusch, Germany

Muellbacher et al. (2003) demonstrated improved hand motor function after plexus anaesthesia in chronic stroke patients. We were interested to see, whether this procedure would also help patients in the subacute phase after stroke ( 4 to 8 weeks after stroke).

We investigated a group of 12 patients after subacute stroke, mean age 64 years $(5 f$ $7 \mathrm{~m})$. All patients had a moderate paresis after their first clinical stroke. Upper plexus anaesthesia was performed on the affected side by an experienced anaesthetist (T.S.) before training started. A special daily training course was implemented for all participants lasting for two weeks. Motor testing was performed by occupational therapists and neurophysiological parameters (motor evoked potentials and somatosensory evoked potentials) were collected before and after therapy. All patients gave informed consent.

A second group of subacute stroke patients with similar demographic and clinical data served as a control group. They received the same special training, but had no upper plexus anaesthesia.

Both, the patients of the experimental group and of the control group showed a clear improvement of arm function during their training. There were no statistical significant differences of overall recovery between the two groups within the two weeks. However, while patients without plexus anaesthesia showed most recovery during the first week, in patients with plexus anaesthesia the speed of recovery was greater in the second week. Furthermore, proximal and distal function showed different time courses of recovery.

The present data suggest, that upper plexus anaesthesia influences the sequence and possibly also the mode of recovery. Our period of observations ( 2 weeks) was however not long enough to show whether the intervention led to a lasting clinical improvement over and above the effect of intensive sensorimotor training.

\section{Recovery and rehabilitation}

THE NATURE OF FATIGUE FOLLOWING STROKE AND TIA

M. Martin, S.M. Walsh, D. Martin, J.A. Harbison

Department of Medical Gerontology, Trinity College Dublin, Dublin, Ireland

Depending on the subgroup studied and assessment tool used fatigue reportedly affects up to $70 \%$ of patients following stroke, Fatigue may be sub-classified as either physical or mental and the term fatigue may include symptoms such as excess daytime sleepiness or somnolence. We sought to define what subjects with stroke and TIA mean when they report 'fatigue'.

Methods: Subjects were recruited from a secondary prevention clinic. All subjects recruited were independent (modified Rankin Score $(\mathrm{mRS})<3$ ) to reduce the confounding effect of physical disability on fatigue scores. Assessment scores were completed for each subject including, the fatigue Severity Score (FSS), Epworth Sleepiness Score (ESS) Abbreviated Mental Test Score (AMTS) and a simple 5 point Lickert scales of frequency of physical and mental fatigue. Fatigue occurring 'often' or 'always' was recorded as significant.

Results: 40 subjects were recruited in the pilot phase $(35 \%$ Male, median age 74years). 9 (23\%) subjects reported significant physical fatigue, and 4 (10\%) mental fatigue. All those reporting mental fatigue also had physical fatigue. Median value for FSS was (3.6, range 1.0-6.6), for AMTS (9, range 6-10) for ESS (4.5 range $0-12)$. FSS correlated significantly with Likert scores for physical fatigue $(r=0.447$, $\mathrm{p}=0.005)$ and mental fatigue $(\mathrm{r}=0.351, \mathrm{p}=0.03)$. FSS correlated with $\mathrm{mRS}(\mathrm{r}=0.388$, $\mathrm{p}=0.021$. Spearman's rho) but Likert fatigue scores did not. No measure of fatigue correlated significantly with ESS or AMTS. ESS however correlated with AMTS $(\mathrm{r}=-0.534, \mathrm{p}<0.001)$.

Conclusion: One quarter of independent subjects with stroke or TIA were found to have significant fatigue which was predominantly physical, rather than mental fatigue. Degree of somnolence was not related to fatigue severity.

\section{Recovery and rehabilitation}

\section{MIRROR MOVEMENTS AFTER STROKE: QUANTIFICATION WITH ACCELEROMETRY AND CORRELATION WITH BRAIN ACTIVATION PATTERNS}

C. Calautti, S.P. Jones, M. Naccarato, N. Sharma, D.J. Day, T.A. Carpenter, E.A. Warburton, J.-C. Baron

Dept. of Clinical Neurosciences, University of Cambridge, Cambridge, United Kingdom

Mirror movements $(\mathrm{MM})$ of the unaffected hand (UH) when moving the affected hand $(\mathrm{AH})$ are well described clinically after stroke, but their neural substrates remain the matter of controversy, with several conflicting hypotheses involving the contralesional M1, ipsilesional M1, both or neither being proposed. In addition, MM during non-effortful movement have not been quantitated using objective means so far. Aims of the present study were to quantify MM during simple index-thumb tapping (IT-taps) using tri-axial accelerometry (TAA), and to study their neural substrates using fMRI.

25 prospectively selected R-handed stroke patients (mean age 63yrs) partially recovered from $\mathrm{L}$ or $\mathrm{R}$ hemiparesis underwent TAA and fMRI during auditory-cued IT-taps at $1.25 \mathrm{~Hz}$. Using TAA over both index fingers we calculated a cross correlation coefficient (CCC) which reflects the occurrence of MM in the opposite hand during IT-taps of the $\mathrm{AH}$ (CCC-AH) and UH (CCC-UH) hand. To investigate their neural substrates, we correlated the $\mathrm{CCC}-\mathrm{AH}$ with the fMRI maps using SPM. Clinical deficit was scored using the European Stroke Scale (ESS), maximum IT-taps in $15 \mathrm{~s}$ (IT-Max) and tapping regularity index (RI). 28 normal right-handed subjects were also studied.

The CCC in controls was $0.065 \pm 0.07$ and $0.075 \pm 0.086$ for the R and L hand, respectively (NS), without significant effects of age. The CCC-AH was significantly $(\mathrm{p} \leq 0.05)$ higher than both the CCC-UH $(0.145 \pm 0.173$ and $0.114 \pm 0.09$, respectively) and the CCC of controls. There was no significant correlation between the CCC-AH and the ESS, time from stroke onset, IT-max and RI. No significant correlation between CCC-AH and the activation maps were found in either the contralesional or ipsilesional hemispheres.

In this study, objective indices of MM during IT-taps were dissociated from both motor deficit and brain activation maps. We argue that MM after stroke could be the result of complex interactions between the individual's built-in attentional system and transcallosal M1 connection strength, the type of task used and the effect of damage from the stroke on the motor system. 


\section{Recovery and rehabilitation}

DISCREPANCY BETWEEN INNER AND OVERT SPEECH: A POTENTIAL FOR MISDIAGNOSIS OF LANGUAGE IMPAIRMENTS IN POST-STROKE APHASIA

S. Geva, E.A. Warburton

University of Cambridge, Cambridge, United Kingdom

Background: Patients with aphasia often complain that there is a poor correlation between the words they think (inner speech) and the words they say (overt speech) A few studies show that patients often attend to their intact inner speech and not their erroneous overt speech, which could explain the frequent observation that many patients do not notice their overt errors. This study tries to characterise the relation between inner and overt speech in post-stroke aphasia and its relation to error monitoring.

Methods: 10 patients (8m, age: $72 \pm 8$ ) with left MCA stroke and problems with speech production were studied at $32 \pm 21$ months post-stroke. Following a structural MRI or CT scan, their language abilities were tested. Next, performance on inner speech tasks, such as homophone and rhyming judgement and recitation, was tested using words, pictures and automatic speech, and compared to overt speech errors.

Results: For two patients inner speech was better preserved than overt speech, one patient had no inner speech, and the rest had similar levels of overt and inner speech. Patients relied on inner speech more than on overt speech for monitoring their speech errors.

Discussion: It is generally assumed that when patients make overt speech errors this is due to their impaired language abilities and hence their subsequent therapy is based on a potential misdiagnosis. However, this study shows that inner speech can remain intact while there is a marked deficit in overt speech. Also, it is suggested that complex tasks using inner speech can be used as an improved therapy technique for patients who cannot speak, potentially leading to improved language abilities. This may have implications for diagnosis, prognosis and therapy of certain patients with post-stroke aphasia.

\section{Experimental studies II. Genetic disorders}

\section{Experimental studies II. Genetic disorders}

AXONAL INJURY DUE TO ISCHAEMIA: PROTECTION OF AXONAL FUNCTION IN A NEW MODEL INDUCED IN THE RAT SPINAL CORD IN VIVO

F. Bei, K.J. Smith

Institute of Neurology, United Kingdom

Background: Ischaemic injury of the central white matter (IICWM) has been relatively little studied despite its contribution to the pathology of ischaemic stroke and vascular dementia. Research has been hampered by a lack of suitable experimental models.

Methods: To develop a new model of IICWM, endothelin-1 (ET-1; 2.3 nmol; a potent vasoconstrictor) was injected bilaterally into the rat spinal grey matter. The local tissue perfusion was continuously monitored by laser Doppler flowmetry. Axonal function in the dorsal columns was assessed by continuously monitoring conduction. KB-R7943 (30 mg/kg; a selective blocker of the reverse-mode operation of the sodium-calcium exchanger) or vehicle was administered intra-arterially 1 hour before the intraspinal injection of ET-1.

Results: Saline/control intraspinal injection had little or no effect on either local tissue perfusion or axonal conduction, but the injection of ET-1 reduced local tissue perfusion by more than $90 \%$ within minutes of application, accompanied by total blockade of axonal conduction. The local tissue perfusion gradually recovered to about $40 \%$ of baseline within 8 hours, and axonal conduction recovered to $18 \%$ of baseline by 4.5 hours after the injection of ET-1. Systemic pre-treatment with KB-R7943 increased the recovery of axonal conduction to $35 \%$ of baseline $(35 \pm 5 \%)$, compared with recovery from vehicle treatment $(12 \pm 9 \%$ of baseline; $\mathrm{P}<0.0001)$. The drug treatment did not diminish the severity of the ischaemia per se. KB-R7943 did not affect the magnitude of axonal conduction in normal, sham-operated rats.

Discussion: A new model of IICWM in vivo has been developed, resulting from the instraspinal injection of ET-1. Treatment with the sodium-calcium exchange blocker KB-7943 may provide a therapeutic approach to protect the function of the central white matter from ischaemic injury.
2 Experimental studies II. Genetic disorders

REDUCED CARDIAC BARORECEPTOR SENSITIVITY IS NOT RELATED TO INCREASED ARTERIAL STIFFNESS FOLLOWING ACUTE STROKE IN TREATED HYPERTENSIVE PATIENTS

N.S. Shah, J. Chernova, R.B. Panerai, J.F. Potter, T.G. Robinson

Ageing \& Stroke Medicine, University of Leicester, Leicester, United Kingdom

Background: Cardiac baroreceptor reflex sensitivity (BRS) is a short-term blood pressure regulator in health and disease state. Cardiac BRS is impaired following ischaemic stroke and predicts long-term death and disability. Impaired cardiac BRS may be due to afferent loop abnormalities due to increased large artery stiffness, impaired central processing of baroreceptor information or efferent loop abnormalities due to impaired blood pressure and heart rate control mechanism following stroke. We evaluated the relationship between large (aortic) artery stiffness and cardiac BRS during the acute phase of ischaemic or haemorrhagic stroke from a subgroup of COSSACS (Continue Or Stop post-Stroke Antihypertensive Collaborative Study).

Methods: Ninety four treated hypertensive stroke patients $(51$ male, mean age $72.4 \pm 12.5$ ) were studied within 48 hours of onset. Cardiac BRS (determined by spectral and sequence analyses) and arterial stiffness estimated by the carotid to femoral pulse wave velocity (PWVcf) using applanation tonometry by SphygmocorTM were obtained. In addition to demographic data, NIHSS (National Institutes of Health Stroke Scale), casual blood pressure (BP) and body mass index were recorded at randomisation.

Results: At baseline, cardiac BRS values were inversely correlated with PWVcf $(\mathrm{r}=-0.24, \mathrm{P}<0.05 ; \mathrm{r}=-0.35, \mathrm{p}=0.003$ for spectral and sequence analysis, respectively). Age was significantly correlated with PWVcf $(r=0.50, p<0.0001)$. In age, baseline NIHSS and mRS adjusted correlation, cardiac BRS was not related to PWVcf. In quantile regression models, taking into account the effect of all cardiovascular variables, and BP values, cardiac BRS was not related to PWVcf at baseline.

Conclusions: Arterial stiffness of the afferent loop involved in the baroreflex arc may account for, at least in part, the reduced cardiac BRS observed in acute stroke patients previously treated with antihypertensives, though age is a strong confounder and nullifies the relationship of cardiac BRS and PWVcf following acute stroke.

\section{Experimental studies II. Genetic disorders}

\section{GENETIC VARIATION IN MEMBERS OF THE LEUKOTRIENE} BIOSYNTHESIS PATHWAY CONFER AN INCREASED RISK OF ISCHAEMIC STROKE - A REPLICATION STUDY IN TWO INDEPENDENT POPULATIONS S. Bevan, M. Dichgans, H. Wiechmann, A. Gschwendtner, T. Meitinger, H. Markus

St Georges, University of London, London, United Kingdom

Background: The recent finding that genetic variants in 5-lipoxygenase activating protein (FLAP) and leukotriene A4 hydrolase (LTA4H) may confer an increased risk of ischaemic stroke has implicated the leukotriene family as potential mediators of cardiovascular disease. Using a case control replication methodology, all members of the leukotriene synthesis pathway and their receptors were examined for genetic variants which may act as risk factors for all ischaemic stroke and stroke subtypes.

Methods: A case control methodology using a UK stroke cohort (872 cases, 933 controls) was adopted, with additional FLAP genotyping and replication of positive findings undertaken in an independent stroke population from Germany (601 cases, 736 controls). 18 variants in FLAP, 2 variants in 5-LO, one variant in LTC4S, 4 variants in LTA4H, 4 variants in CYSLTR1, 2 variants in CYSLTR2 and 5 variants spanning LTB4R and LTB4R2 were examined in the UK cohort. Replication of variants in the two genes in which associations were detected in the UK population (LTC4S and LTB4R) was performed in the German cohort.

Results: Associations were identified with variants in FLAP, leukotriene C4 synthase (LTC4S) and the leukotriene B4 receptor (LTB4R1/2) complex. Differing risks were identified for ischaemic stroke subtypes. A variant in LTC4S was found to confer a 1.5 fold increase in risk of small vessel disease (RR 1.515 1.041-2.262 $\mathrm{p}=0.043$ ) with replication in an independent cohort showing a similar risk (RR $1.687,1.065-2.675, \mathrm{p}=0.026$ ). A haplotype in the LTB4R $1 / 2$ complex was found to confer a 2.3 fold increase in risk of cardioembolic stroke (RR 2.118, 1.194-3.760, $\mathrm{p}=0.01$ ) and replication in a German cohort revealed a similar risk with a second distinct haplotype (RR 2.060, 1.162-3.665, $\mathrm{p}=0.013$ ).

Conclusions: Genetic variation in leukotriene pathway members and their receptors confer an increased risk of ischaemic stroke in two independent populations. These risks show different magnitudes depending on ischaemic stroke subtype. 


\section{Experimental studies II. Genetic disorders}

NEUROPROTECTION AFTER ISCHAEMIC PRECONDITIONING IS MEDIATED BY TLR4

J.M. Pradillo, I. García-Yébenes, D. Fernández-López, J. Caso, O. Hurtado, M. Sobrado, M.A. Moro, I. Lizasoain

Department Pharmacology, School of Medicine, Universidad Complutense de Madrid, Spain

Background: It has been demonstrated that a short ischaemic event (ischaemic preconditioning; IPC) can result in a subsequent resistance to severe ischaemic injury (ischaemic tolerance; IT). We have recently demonstrated the role of immunity and in particular TLR4 in brain ischaemia (Caso et al., Circulation 2007). We have now used an in vivo model of IPC to investigate whether TLR4 signalling is involved in IT.

Methods: Experiments were carried out on TLR4-deficient mice (C57BL/10ScNJ) and its respective control (C57BL/10ScSn). A period of 10-min temporary middle cerebral artery occlusion (tMCAO) was used for focal IPC, whereas sham surgery was used for control group. To evaluate the ability of IPC to induce IT, permanent MCAO was performed $48 \mathrm{~h}$ after IPC. Stroke outcome was evaluated by determination of infarct volume and assessment of neurological scores. Brains were collected $24 \mathrm{~h}$ after stroke and infarct volume was measured by TTC staining. 24h after IPC, MMP9, iNOS expression and NF-kB activation were studied by western blot and by ELISA kit (determining p65 subunit in the cellular nucleus).

Results: IPC produced a reduction in infarct volume and a reduction on moto deficit measured by a neurological test in mice expressing TLR4 normally ( $\mathrm{ScSn}$ : SHAM+MCAO: $35.4 \pm 3$ vs IPC+MCAO: $13.9 \pm 2 \mathrm{~mm}^{3} ; 60 \%$ reduction, $\mathrm{P}<0.05$ ). However, TLR4-deficient mice had significantly smaller reduction in infarct volume when compared with control mice (ScNJ: SHAM+MCAO: $28.2 \pm 2$ vs IPC+MCAO: $23.1 \pm 2 \mathrm{~mm}^{3} ; 18 \%$ reduction, $\mathrm{P}<0.05$ ). Western blot analysis of iNOS and MMP9 showed an upregulation in the expression of these proteins in both substrain mice, being higher in mice with TLR4 $(\mathrm{ScSn})$. Same results were observed in the NF-кB activation

Conclusions: These data demonstrate that TLR4 signalling is involved in brain tolerance. The difference in the percentage of decrease in infarct volume produced by IPC between $\mathrm{ScSn}$ and $\mathrm{ScNJ}$ (60\% vs. 18\%), and the higher expression of iNOS, MMP9 and NF-kappaB activation in mice expressing TLR4, clearly supports these conclusions.

\section{Experimental studies II. Genetic disorders}

IMPLANT FOR AUGMENTATION CBF CLINICAL TRIAL-1 (IMPACT-1). SAFETY AND EFFICACY OF THE ISCHEMIC STROKE SYSTEM (ISS*) IN THE TREATMENT OF ACUTE ISCHEMIC STROKE: AN INTERIM ANALYSIS N. Borenstein, D. Khurana, S. Kaul, A. Csányi, N. Ichaporia, D. Schneider, D. Tanne, Y. Solberg, M. Fisher

Tel Aviv Medical Center, Tel Aviv, Israel

Background: In rat stroke models, sphenopalatine ganglion (SPG) stimulation up to 24 hours after stroke onset augments cerebral blood flow, reduces infarct volume and improves neurological deficits. Presented are preliminary safety and efficacy data of SPG stimulation with ISS in patients with acute ischemic stroke (AIS).

Design: This is an ongoing multi-center, multi-national open label study recruiting patients with AIS in the anterior vasculature, NIHSS 7-20, treatment initiated within the first 24 hours following onset. The ISS is implanted adjacent to the SPG with a minimally invasive oral procedure (15 min., local anesthesia). The therapeutic regimen consists of $3 \mathrm{hr} / \mathrm{d}$ stimulation up to 7 days. Primary endpoint is incidence of $\mathrm{AE}$ and SAE, secondary is the efficacy of SPG stimulation as measured by mRS and NIHSS at day 90. Efficacy results are compared to an historical control population composed of the placebo group in two large previous AIS trials, the NINDS rt-PA studies I and II.

Results: To date 62 patients have been enrolled. A small number of AE and SAE were observed in a few patients, none of them deemed related to the treatment. The mean 90 day mRS score of the Intend To Treat (ITT) patients cohort in the study is 2.53 and that of the Per Protocol (PP) patients cohort is 2.31, both of which are significantly lower than the mean 90 day $\mathrm{mRS}$ of the matched NINDS controls, which is 3.47 ( $\mathrm{CMH}$ test $\mathrm{p}=0.0031$ and $\mathrm{p}=0.0006$, respectively). Moreover, both ITT and PP cohorts have a significantly higher rate of favorable NIHSS outcome as compared to the controls (ITT: $50 \%$ and PP: $56.3 \%$ vs. $23.6 \%$ for the NINDS controls, $\mathrm{p}=0.0015$ and $\mathrm{p}=0.0002$ respectively, using Chi square).

Conclusion: This interim analysis suggests that SPG stimulation appears to be safe and efficacious for the treatment of AIS when initiated within $24 \mathrm{hr}$ after symptom onset. ImPACT-1 is still enrolling. In 2008 a larger multi-national multi-center pivotal study (ImPACT-24) is planned to commence.

*Investigational device limited by Federal law to investigational use.
6 Experimental studies II. Genetic disorders

\section{ADDITIVE EFFECTS OF STATIN AND DIPYRIDAMOLE ON CEREBRAL BLOOD FLOW AND STROKE PROTECTION}

H.H. Kim, N. Sawada, H.S. Lee, Z. Zhou, M.A. Moskowitz

Brigham \& Women's Hospital and Harvard Medical School, Cambridge, MA, USA

Recent studies suggest that dipyridamole (DP) may exert stroke protective effects beyond platelet inhibition. The purpose of this study is to determine whethe statin and dipyridamole (DP) could enhance stroke protection through nitric oxide (NO)-dependent vascular effects. Mice were pre-treated with DP (10-60 mg/kg, q12 $\mathrm{hr}, 3 \mathrm{~d}$ ) alone or in combination with a statin (simvastatin, 0.1 to $20 \mathrm{mg} / \mathrm{kg} / \mathrm{d}, 14 \mathrm{~d}$ ) before transient intraluminal middle cerebral artery occlusion. Although simvastatin $(1 \mathrm{mg} / \mathrm{kg} / \mathrm{d}, 14 \mathrm{~d})$ increased endothelial NO synthase (eNOS) activity by $25 \%$ and DP $(30 \mathrm{mg} / \mathrm{kg}, \mathrm{q} 12 \mathrm{hr}, 3 \mathrm{~d})$ increased aortic cGMP levels by $55 \%$, neither statin nor DP alone, at these sub-therapeutic doses, increased absolute cerebral blood flow (CBF) or conferred stroke protection. However, the combination of sub-therapeutic doses of simvastatin and DP increased CBF by $50 \%$, decreased stroke volume by $54 \%$, and improved neurological motor deficits, all of which were absent in eNOS-/- mice. In contrast, treatment with ASA (10 mg/kg/d, 3 d) did not augment the neuroprotective effects of DP. These findings indicate that statin and DP exert additive NO-dependent vascular effects. These results suggest that the combination of statin and DP may have greater benefits in stroke protection than statin alone through vascular protection.

\section{Experimental studies II. Genetic disorders}

\section{STATINS MIGHT HELP CLEARANCE OF EXCESS EXTRACELLULAR GLUTAMATE IN STROKE}

T. Gasull, V. Guirao, J. Ponce, A. Davalso

Fundacio Institut Investigacio Ciencies Salut Germans Trias i Pujol, Badalona, Spain

Background: Excitotoxicity by overactivation of the N-methyl-D-aspartate (NMDA) subtype of glutamate receptors initiates damage in stroke. The widely prescribed drugs statins have been reported to protect from excitotoxicity in neurons in culture, and we recently found that simvastatin reduce the association of NMDA receptors to the membrane functional domains called lipid rafts without altering its total expression. However, the effect of statins on the other players of the excitotoxic game still remains to be elucidated. To provide new light into this subject we studied the effect of simvastatin on the expression and the association to lipid rafts of the neuronal glutamate transporter 3 (EAAT3) and its negative regulator, the glutamate transport associated protein (GTRAP).

Methods: Primary neuronal cultures were treated with simvastatin for 5 days, total protein and protein of the lipid rafts fractions from control and simvastatintreated neurons were obtained, and Western blots were performed using specific anti-EAAT3 and anti-GTRAP antibodies.

Results: Sustained treatment with simvastatin increased EAAT3 total expression and decreased the total expression of GTRAP (Figure 1). However, simvastatin did not change the percentage of EAAT3 or GTRAP associated to lipid rafts.

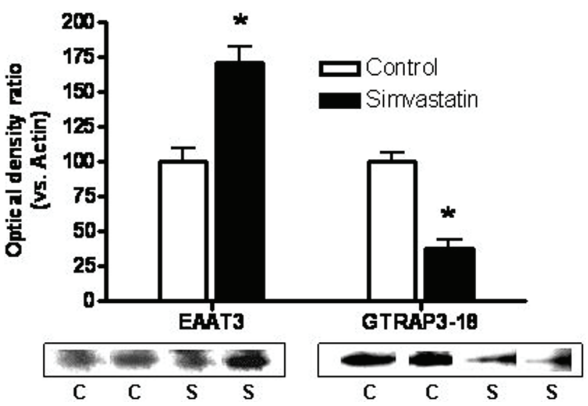

Figure 1

Discussion: Pre-treatment with simvastatin increases the expression of the neuronal glutamate transporter and decreases the expression of its inhibitor. Therefore, by improving the capacity of neurons to uptake glutamate, statins might reduce excitotoxicity during stroke. This effect might underlay the benefits reported for stroke patients in statins before the onset of cerebral ischemia. 
8 Experimental studies II. Genetic disorders

MAXIMIZING NEUROPROTECTION BY MINOCYCLINE IN CELL CULTURE AND FOLLOWING INTRACEREBRAL HEMORRHAGE IN MICE M. Xue, E. Mikliaeva, V.W. Yong

Hotchkiss Brain Institute and University of Calgary, Calgary, AB, Canada

The brain injury following intracerebral hemorrhage (ICH) involves inflammatory responses and neuronal death. Minocycline (MO) has anti-inflammatory and anti-apoptotic properties, and it provides histological protection in $\mathrm{ICH}$, although contradictory results have also been noted due to the dose and route of administration used for MO. We hypothesize that high doses of MO applied locally to the proximity of ICH would be more efficacious.

In culture, human fetal neurons (HFN) were exposed to human blood, and immunostaining was used to examine neuronal survival. In mice, $10 \mu 1$ of autologous blood mixed with MO or saline was injected into the right striatum of CD-1 mice. Other mice received MO injected intracerebrally (IC) into the hematoma but treatment was initiated $1 \mathrm{~h}$ after ICH. ICH mice injected intraperitoneally (IP) with $\mathrm{MO}$ was used as comparison group. $24 \mathrm{~h}$ after $\mathrm{ICH}$, brain were evaluated for lesion areas, neuronal death and inflammation. Other groups of mice survived 2 weeks for behavioral tests.

In culture, concentration-dependent neurotoxicity was observed when HFN were exposed to blood. MO added $5 \mathrm{~min}$ before blood reduced its neurotoxicity and there was a concentration-dependent effect from 10 to $40 \mathrm{mg} / \mathrm{ml}$ of MO. In vivo, $24 \mathrm{~h}$ following $\mathrm{ICH}$, the histopathological changes were significantly reduced by MO applied IC plus IP compared to each separate route of administration. Moreover, the reduction of ICH-inflicted brain damage and neuronal death was achieved by $\mathrm{MO}$ in a concentration-dependent manner when MO was applied IC. Behavioral tests over $14 \mathrm{~d}$ show significant improvement in mice treated with MO applied IC plus IP compared to IP administration.

Our results have relevance to the application of MO in humans to maximize recovery from certain neurological insults.

\section{Experimental studies II. Genetic disorders}

\section{MITOCHONDRIAL HAPLOGROUP H1 IS PROTECTIVE FOR ISCHEMIC} STROKE

A. Rosa, B.V. Fonseca, T. Krug, H. Manso, L. Gouveia, I. Matos, G. Lopes, J.M. Ferro, A.M. Vicente, S.A. Oliveira, and the PORTuguese Stroke GENetics (PORTSGEN) Group

Instituto Gulbenkian de Ciência, Oeiras, Portugal

Background: The genetic contribution to stroke is well established but it has proven difficult to identify the genes and the disease-associated alleles mediating this effect, possibly because only nuclear genes have been intensely investigated so far. Mitochondrial DNA (mtDNA) has been implicated in several disorders having stroke as one of its clinical manifestations. The aim of this case-control study was to assess the contribution of mitochondrial polymorphisms and haplogroups to ischemic stroke risk and age-at-onset.

Methods: We genotyped 28 mtDNA single nucleotide polymorphisms (SNPs) defining the major European haplogroups in 534 ischemic stroke patients and 499 controls collected in Portugal, and tested their allelic and haplogroup association with ischemic stroke risk and age-at-onset.

Results: Haplogroup H1 was found to be significantly less frequent in stroke patients than in controls $(\mathrm{OR}=0.61,95 \% \mathrm{CI}=0.45-0.83, \mathrm{p}=0.005)$, when comparing each clade against all other haplogroups pooled together. Conversely, the pre$\mathrm{HV} / \mathrm{HV}$ and $\mathrm{U} \mathrm{mtDNA}$ lineages emerge as potential genetic factors conferring risk for stroke $(\mathrm{OR}=3.14,95 \% \mathrm{CI}=1.41-7.01, \mathrm{p}=0.004$, and $\mathrm{OR}=2.87,95 \% \mathrm{CI}=1.13$ 7.28, $\mathrm{p}=0.022$, respectively). SNPs 3010GA, 7028CT and 11719GA strongly influence ischemic stroke risk, their allelic state in haplogroup $\mathrm{H} 1$ corroborating its protective effect. None of the tested haplogroups or polymorphisms was found to strongly associate with the age-at-onset of ischemic stroke.

Discussion: Our data suggests that mitochondrial haplogroup $\mathrm{H} 1$ has an impact on ischemic stroke risk, but mtDNA does not influence its age-at-onset.
10 Experimental studies II. Genetic disorders

NXY-059 EFFICACY META-ANALYSIS IN INDIVIDUAL ANIMALS WITH STROKE (NEMAS)

P.M.W. Bath, L.J. Gray, A.R. Green, For NEMAS Collaborators Institute of Neuroscience, University of Nottingham, Nottingham, United Kingdom

Background: NXY-059 was neuroprotective in experimental stroke but ineffective in a large clinical trial. In the first ever meta-analysis using individual animal data, we assessed preclinical stroke studies of NXY-059.

Methods: Studies from AstraZeneca (AZ) and PubMed searches. Individual data for each animal (or published summary if unavailable) were obtained from the lead author of each study and/or AZ. Multilevel models compared NXY-059 with control taking account of heterogeneity between trials. Infarct volume and neurological impairment were standardised to reflect different species and scales. Standardised mean difference (SMD) and 95\% confidence intervals (95\% CI) are presented.

Results: 21 studies (11 laboratories) involved 534 animals (NXY-059 322, control 212) from 3 species (mice 9, rats 489, marmosets 36). 7 studies were unpublished (6 were neutral). Individual data were available for 445 animals. Investigators from 4 studies (89 animals), all academic, did not share data. Studies variably used randomisation $(8,38 \%)$, and blinding to treatment of the surgeon $(7,33 \%)$ and outcome assessor $(10,48 \%)$. NXY-059 was associated with reductions in total (SMD -1.2, 95\% CI -1.5 to -0.8 ), cortical (SMD -1.1, 95\% CI -1.5 to -0.7) and subcortical $(-1.3,95 \%$ CI -1.6 to -0.9$)$ lesion volume; efficacy was seen in models of transient and permanent ischaemia, up to 180 minutes post occlusion. 18 $(7 \%)$ animals receiving NXY-059 had no lesion versus receiving control $(\mathrm{p}=0.001)$. Neurological impairment was reduced with NXY-059 (SMD -1.1, 95\% CI -1.3 to -0.8). There was no difference in death rate.

Conclusion: NXY-059 reduced lesion volume and impairment in experimental stroke models. Absence of lesions in some animals given NXY-059 could reflect neuroprotection or bias. Preclinical stroke studies must embody the principles of clinical trials (randomisation, blinding), and be followed by a meta-analysis using individual animal data.

Oral Session 